\title{
Characterization of Halftone Prints based on Microscale Image Analysis
}

\author{
Mahziar Namedanian
}

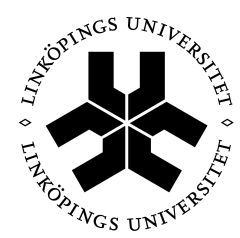

\section{Linköping University \\ INSTITUTE OF TECHNOLOGY}

Department of Science and Technology

Linköping University, SE-601 74, Norrköping, Sweden

Norrköping 2013 


\title{
Characterization of Halftone Prints based on Microscale Image Analysis
}

\author{
(C)Mahziar Namedanian, 2013 \\ mahna@itn.liu.se
}

Image Reproduction and Graphic Design

Department of Science and Technology Campus Norrköping, Linköping University SE-601 74 Norrköping, Sweden

ISBN 978-91-7519-499-8

ISSN 0345-7524

Printed by LiU-Tryck, Linköping, Sweden, 2013 
To my lovely little

Nila 



\section{Abstract}

Ink spreading and lateral light scattering in the substrate affect the color of a halftone print. One of the most important phenomena which affects the print result is dot gain, meaning that printed dots appear larger than the dots in the digital bitmap. This is partly due to the ink spreading and ink penetration into the substrate, resulting in an enhancement of the physical dot size, referred to as the physical dot gain. Lateral propagation of light in paper, causes printed dots to appear larger than their physical size, which is called optical dot gain. Characterization of total dot gain, i.e. the combination of physical and optical dot gain, is an important issue in the study of paper properties and print characteristics. Many models based on macroscopic measurements are reported in the literature to separately characterize both physical and optical dot gains. The aim of this study is to go beyond the macroscopic models, and to study the halftone prints on a microscopic scale, by using microscale images captured by a high-resolution camera.

In this dissertation, three approaches based on the Murray-Davies model are proposed to obtain the total dot gain. In the first approach, by minimizing the root-mean-square difference between the calculated spectrum and the reflected spectrum measured by the spectrophotometer, the total dot gain is approximated. The other two approaches are based on microscale images captured by a high-resolution camera. These two approaches differ in their schemes on how to obtain the gray tone of the full tone ink. By the use of microscale images, it is also possible to illustrate the shape of the effective dot area for the investigated paper substrate.

A novel approach based on the histogram of microscale images is also proposed to separate physical from optical dot gain. Attaining the physical dot gain characteristic makes it possible to determine the actual physical dot shape, by which the Modulation Transfer Function (MTF) of the paper substrate is estimated. The proposed approach is validated by comparing the estimated MTF of eleven offset printed coated papers to the MTF obtained from the unprinted papers using measured and Monte-Carlo simulated edge response.

Another potential usage based on the separation of physical from optical dot gain, is to study the characterization of different color inks. 
In this dissertation, the dependency of dot gain and wavelength in color print is investigated. It has been illustrated that the light scattering effect, which is the reason for optical dot gain creation, must be less sensitive to different wavelength bands. It has also been shown that it is possible to separate two printed color inks by illuminating the halftone print with having light in the reflective wavelength band of one of the two colors.

Comparison of the optical dot gain for different dot shapes and perimeters, but with the same area, shows the dependency of optical dot gain on the dot shape perimeter. The dependency of optical dot gain on the dot shape perimeter verifies the fact that the amount of optical dot gain is different for different types of halftoning. 


\section{Acknowledgement}

I would have not been able to write this dissertation without the support and help I received from the wonderful people around me, and unfortunately, it is not possible to name and mention all of them here.

My deepest gratitude goes to my supervisor, Dr. Sasan Gooran. Without his support, continuous guidance, incredible patience and encouragement throughout my study and research, I would have never been able to accomplish this work. Of course, I am also grateful for his friendship, which has been invaluable on both academic and personal level.

I would like to express my great appreciation to Prof. Björn Kruse, whom his kind personality has been an inspiration, for accepting me to join his group. Also special thanks goes to Dr. Daniel Nyström and Dr. Ludovic Coppel for their valuable discussions and informative meetings, which resulted in some collaborative publications.

I would like to acknowledge the financial support of the Swedish Governmental Agency for Innovation Systems (VINNOVA). I am also thankful to all the people in the PaperOpt project, which I was fortunate to be involved in. Especial thanks to Prof. Per Edström for managing useful meetings and collaborations between different sections and bringing up useful discussions. I am grateful to Dr. Petter Kolseth for his positive outlook, confidence in my research and for sharing his valuable experimental techniques, that inspired me a lot.

I would like to thank all my colleagues at the division of Media Information Technology (MIT). Especial thanks goes to my sincere colleague, Dr. Yuan Yuan $\mathrm{Qu}$, for her extreme kindness and incredible friendship. I also want to show my appreciation to Mrs. Gun Britt Löfgren for all her help and support during all these years. Thanks to Lei and Paula for all the enjoyable coffee-breaks and lunches.

My deepest appreciation goes to my incredible family and wonderful friends for their encouragement and supporting me in every way possible throughout my life.

Finally, and most importantly, I can never thank my lovely wife, Sara, enough, for all her love and support. I am sure this $\mathrm{PhD}$ is a result of our happy marriage! $\varnothing$

Norrköping, November 2013

Mahziar Namedanian 


\section{Abbreviations}

$\begin{array}{ll}\text { AM } & \text { Amplitude Modulation } \\ \text { CCD } & \text { Charge Coupled Device } \\ \text { CIE } & \text { Commission Internationale de l'Eclairage } \\ \text { CIELAB } & \text { CIE } L^{*} a^{*} b^{*} \text { color space and color appearance model } \\ \text { CIEXYZ } & \text { CIEXYZ color space and color appearance model } \\ \text { CMYK } & \text { Cyan-Magenta-Yellow-Black } \\ \text { DPI } & \text { Dots Per Inch [Dots/inch] } \\ \text { ESF } & \text { Edge Spread Function } \\ \text { FM } & \text { Frequency Modulation } \\ \text { FWHM } & \text { Full Width at Half Maximum } \\ \text { ISO } & \text { International Organization for Standardization } \\ \text { JND } & \text { Just Noticeable Difference } \\ \text { KM } & \text { Kubelka-Munk } \\ \text { LPI } & \text { Lines Per Inch [Lines/inch] } \\ \text { LSF } & \text { Line Spread Function } \\ \text { MC } & \text { Monte-Carlo } \\ \text { MIH } & \text { Microscale Image Histogram } \\ \text { MTF } & \text { Modulation Transfer Function } \\ \text { OTF } & \text { Optical Transfer Function } \\ \text { PSF } & \text { Point Spread Function } \\ \text { RGB } & \text { Red-Green-Blue } \\ \text { RMS } & \text { Root Mean Square } \\ \text { RT } & \text { Radiative Transfer } \\ \text { SPD } & \text { Spectral Power Distribution } \\ \end{array}$




\section{Contents}

1 Introduction 1

1.1 Introduction . . . . . . . . . . . . . . . . 3

1.2 Scope of the Dissertation . . . . . . . . . . . 4

1.3 Contributions . . . . . . . . . . . . . . . 4

1.4 Publications . . . . . . . . . . . . . . . 6

1.5 Dissertation Outline . . . . . . . . . . . . 7

2 Color Fundamentals $\quad 9$

2.1 Introduction . . . . . . . . . . . . . . . . 11

2.2 Color Observation . . . . . . . . . . . . . . 11

2.2 .1 CIE Standard Illuminant . . . . . . . . . . . 12

2.2 .2 CIE Standard Observer . . . . . . . . . . . . . . . 13

2.2.3 CIE Color Matching Functions . . . . . . . . . 15

2.3 CIE Color Spaces . . . . . . . . . . . . . . . . . 16

2.3.1 CIEXYZ Color Space . . . . . . . . . . . 17

2.3.2 CIELAB Color Space . . . . . . . . . . . . . 18

2.3.3 Color Difference Equations . . . . . . . . . . . . 19

2.4 Color Printing . . . . . . . . . . . . . . . . . 21

2.4.1 Color Mixing . . . . . . . . . . . . . . . . . . . 21

2.4 .2 Color Printing Methods . . . . . . . . . . . 25

3 Halftone Color Reproduction 27

3.1 Introduction . . . . . . . . . . . . . . . . . . 29

3.2 Digital Halftoning . . . . . . . . . . . . . . . . . . . 29

3.2.1 AM and FM Halftoning . . . . . . . . . . . 31

3.2.2 FM Second Generation Halftoning . . . . . . . . 32

3.2 .3 Color Halftoning . . . . . . . . . . . . . . . . . . 34

3.3 Dot Gain . . . . . . . . . . . . . . . . 35

3.3.1 Physical Dot Gain . . . . . . . . . . . 36 
3.3 .2 Optical Dot Gain . . . . . . . . . . . . . . . 36

3.4 Modeling Halftone Color Reproduction . . . . . . . . . . 38

3.4.1 Murray-Davies Model . . . . . . . . . . . . . . . . 39

3.4.2 Yule-Nielsen Model . . . . . . . . . . . . . . . . . . 41

3.4.3 Expanded Murray-Davies Model . . . . . . . . . . 43

3.4 .4 Neugebauer Model . . . . . . . . . . . . . . . . . . 43

3.4.5 Yule-Nielsen Modified Neugebauer Model . . . . . 45

3.4.6 Clapper-Yule Model . . . . . . . . . . . . . . . . . 46

3.4.7 Kubelka-Munk Model . . . . . . . . . . . . . . . . 47

3.4.8 Monte-Carlo Simulations . . . . . . . . . . . 50

4 Material and Measurement $\quad 51$

4.1 Introduction . . . . . . . . . . . . . . . 53

4.2 Spectral Measurement . . . . . . . . . . . . . . 54

4.3 Image Acquisition System . . . . . . . . . . . . . . . 54

4.3.1 Image Acquisition Setup . . . . . . . . . . . . . 54

4.3 .2 Color Filters . . . . . . . . . . . . . . 56

4.3.3 Gray Scale Image Acquisition . . . . . . . . . . . . 58

4.3 .4 Color Image Acquisition . . . . . . . . . . . . . 59

4.3.5 Multi-Channel Image Acquisition . . . . . . . . . . 59

4.4 Test Targets and Measurements . . . . . . . . . . . . 62

4.4 .1 Test Target $1 \ldots \ldots \ldots \ldots$. . . . . . . . . 62

4.4 .2 Test Target $2 \ldots \ldots \ldots \ldots$. . . . . . . . . 64

4.4 .3 Test Target $3 \ldots \ldots \ldots$. . . . . . . . . . 64

4.5 Paper Type . . . . . . . . . . . . . . . . 65

5 Microscale Analysis of Halftone Print $\quad 67$

5.1 Introduction . . . . . . . . . . . . . . . 69

5.2 Image Histogram . . . . . . . . . . . . . . . . . 69

5.2.1 Comparison of Reflected and Transmitted Image Histogram . . . . . . . . . . . . . . 71

5.3 Estimation of Total Dot Gain _ . . . . . . . . . 73

5.3 .1 Using Spectrophotometer . . . . . . . . . . . 73

5.3.2 Microscale Image Approach . . . . . . . . . . . . 74

5.4 Estimation of Physical Dot Gain . . . . . . . . . . . . 79

5.4.1 Arney et al. Approach . . . . . . . . . . . . . 80

5.4.2 Microscale Image Histogram Approach . . . . . . 81

5.5 Summary and Discussion . . . . . . . . . . . 85 
6 MTF Characterization of Paper $\quad 87$

6.1 Introduction . . . . . . . . . . . . . . . . . 89

6.2 Reflection Image Model . . . . . . . . . . . . . . . . 90

6.3 MTF Characterization by MIH Approach . . . . . . . . . 91

6.4 MTF Measured by Knife-Edge Method . . . . . . . . . . . 95

6.5 MTF Simulated by Monte-Carlo . . . . . . . . . . . . 96

6.6 MTF Study of Microscale Transmitted Images _. . . . . 97

6.7 Validity Evaluation of MTF Models . . . . . . . . . . . 98

6.8 Summary and Discussion . . . . . . . . . . . . . 101

7 Characterizing Dot Gain for Different Dot Shapes 103

7.1 Introduction . . . . . . . . . . . . . . . 105

7.2 Comparison of Optical Dot Gain for Different Dot Shapes 105

7.3 Ink and Paper Interaction . . . . . . . . . . . . . . . . 107

7.4 Dot Gain and Halftoning Techniques . . . . . . . . . . 111

7.5 Color Gamut Determination . . . . . . . . . . . . . . 117

7.5.1 Gamut Volume . . . . . . . . . . . . . . . 119

7.6 Summary and Discussion . . . . . . . . . . . . . . 119

8 Characterization of Color Halftone Print 121

8.1 Introduction . . . . . . . . . . . . . . . 123

8.2 Primary and Secondary Ink Spectra . . . . . . . . . . 123

8.3 Separation of Inks in Color Print . . . . . . . . . . . 125

8.3.1 Omitting the Shadow Effect . . . . . . . . . . . 129

8.4 Wavelength Dependency of Dot Gain in Color Print . . . 131 8.4.1 Wavelength Dependency of Light Scattering . . . 135

8.5 Register Shift in Color Prints . . . . . . . . . . . . . . 138 8.5.1 Register Shift Measurements . . . . . . . . . . . 140

8.6 Summary and Discussion . . . . . . . . . . . 142

9 Summary and Future Work 145

9.1 Summary . . . . . . . . . . . . . . . . . . . 147

9.2 Future Work . . . . . . . . . . . . . . . . . . 148

$\begin{array}{ll}\text { Bibliography } & 151\end{array}$ 


\section{Chapter 1}

\section{Introduction}

Contents

1.1 Introduction $\ldots \ldots \ldots \ldots \ldots \ldots$

1.2 Scope of the Dissertation . . . . . . . . . 4

1.3 Contributions . . . . . . . . . . 4

1.4 Publications . . . . . . . . . . 6

1.5 Dissertation Outline . . . . . . . . . 7 


\subsection{Introduction}

The demands on the quality of image and color reproduction are increasing day by day, which results in advancement of the study in halftoning techniques, paper and ink properties. Characterization of paper, light scattering in halftone prints, and inks' behaviors are crucial for the graphic arts and paper industries, and play a significant role in color reproduction and image quality.

Printed media such as books, brochures, magazines, and newspapers are still one of the most common ways of communication. Although most information is nowadays available online and can be read through screens, the variety of printing products is not declining. The demand for high print quality requires more accurate ways of judging the print quality. It is important for the printing industry to be able to answer for the accuracy of the image quality.

Analyzing the print quality gives a good intuition to the study of system calibration of printing devices. For calibrating printing devices, it is required to find a relation between the digital input and the print results. This relationship is highly non-linear and depends on many different parameters, such as spectral reflectance of the inks, paper properties, halftoning techniques, ink spreading, light scattering, etc.

One of the most important phenomena that affects the print quality is dot gain, meaning that printed dots appear larger than the dots in the digital bitmap. The term dot gain refers to a combination of physical and optical dot gain. The physical dot gain is partly due to the ink spreading and ink penetration into the substrate, that results in an enhancement of the physical dot size. The optical dot gain originates from lateral propagation of light in paper, that also causes printed dots to appear larger than their physical size. An accurate determination of physical dot gain is useful for correcting tone reproduction, investigating on ink behavior, and quantitatively evaluating the effect of optical dot gain. Due to their different intrinsic nature, in order to accurately model the print results, physical and optical dot gains need to be analyzed, separately. However, in the output of the measurement devices, the physical and optical dot gains always co-exist. Hence, the separation of the two dot gains is a complicated task, and one of the major studies in the area of print analysis.

In this dissertation the halftone color prints are analyzed in microscopic scale. The microscopic scale analysis reveals properties of halftone 
color prints, which can not be derived by conventional macroscopic color measurements. Using microscale images of halftone print, captured by a high-resolution camera, allows us to study the halftone dot shapes, ink spreading, and light scattering effect in micro scale.

\subsection{Scope of the Dissertation}

Most available models for analyzing the outcome of the print results are based on macroscopic measurements, giving the average value over an area that is relative to the halftone dot size. The aim of this dissertation is to go beyond the macroscopic models and to study the print properties on a microscopic level. An accurate image acquisition system is required to capture the images in the microscopic level. In this study, a highresolution camera is used to capture the microscale images. The use of a high-resolution camera makes it possible to clearly observe the individual halftone dots and their surroundings.

In this research dissertation, a novel approach is provided to separate the physical from optical dot gain. By determining the actual physical dot shape, it is possible to investigate the dependency of ink spreading and light scattering effect on the shape of the printed dots. Moreover, the mentioned approach can be used to estimate the Modulation Transfer Function (MTF), which represents one of the optical properties of paper, i.e. light scattering in the paper.

In this dissertation, a method is presented to separate color inks from each other. By separating the color inks, it is possible to study the properties of each primary color ink. Comparing the difference between the properties gives more insight to the characteristics of the color inks in different color printing situations.

\subsection{Contributions}

The main contributions of this dissertation can be summarized as follows.

1. Characterization of total dot gain (physical and optical) by microscale image analysis. Three approaches based on Murray-Davies model are presented and evaluated to estimate the total dot gain. One of the approaches is based on macroscopic measurement, using reflectance spectrum obtained by the spectrophotometer and the 
other two are based on microscopic measurement using reflected microscale images captured by the high-resolution camera.

2. A comprehensive study on the histograms of the reflected and transmitted microscale images. This comparison shows that although the transmitted image includes less optical dot gain compared to the reflected image, the transmittance also incorporates small amount of optical dot gain.

3. Introducing a novel approach to separate physical from optical dot gain by using the histogram of microscale images. The proposed approach chooses a threshold as the border between dots and paper, by finding the minimum value of the histogram between the two peaks corresponding to the reflectance values of the ink and paper between ink dots.

4. Estimating the Modulation Transfer Function (MTF) for eleven offset printed coated papers and comparing it with the MTFs obtained from unprinted papers using measured (knife-edge method) and Monte-Carlo simulated edge response.

5. Comparison of optical dot gain for different dot shapes, which shows the dependency of optical dot gain on the dot shape perimeter. However, there is a limit to the ratio of dot perimeter to dot area at which the optical dot gain is saturated.

6. Evaluation of the FM second generation halftoning technique (developed by our research group at Linköping university) in terms of optical dot gain, ink behavior, and color gamut compared to the AM and FM first generation halftoning techniques. All the investigations have been applied and compared for two types of paper: coated and uncoated.

7. Presenting a method to separate color inks from each other by using a number of color filters.

8. Comparing the optical dot gain for black ink at different wavelength bands shows that the light scattering effect, which is the reason for optical dot gain creation, must be less sensitive to different wavelength bands.

9. Introducing a novel approach based on image processing to measure the register shift of printing devices. 


\subsection{Publications}

Most parts of the material presented in this dissertation have previously appeared in journals and conference proceedings. Here is the list of author's publications used in this dissertation and sorted in chronological order.

- S. Gooran, M. Namedanian, and H. Hedman, "A New Approach to Calculate Color Values of Halftone Prints", Proceedings of IARIGAI $36^{\text {th }}$ Research Conference, Advances in Printing and Media Technology, 2009.

- M. Namedanian and S. Gooran, "High Resolution Analysis of Optical and Physical Dot Gain", Proceedings of TAGA-Technical Association of the Graphic Arts, pp. 48-51, 2010.

- M. Namedanian and S. Gooran, "Characterization of Total Dot Gain by Microscopic Image Analysis", Journal of Imaging Science and Technology, vol. 55, pp. 040501-1-040501-7, 2011.

- M. Namedanian, S. Gooran, and D. Nyström, "Investigating the Wavelength Dependency of Dot Gain in Color Print", Proceedings of IS\&T/SPIE, Electronic Imaging Science and Technology, vol. 7866, pp. 786617-1-786617-8, 2011.

- M. Namedanian and S. Gooran, "Characteristic Analysis of the Primary Color Inks in Color Print", Proceedings of IARIGAI, $38^{\text {th }}$ Research Conference, Advances in Printing and Media Technology, pp. 317-322, 2011.

- S. Gooran, D. Nyström, M. Namedanian, and S. Hauck, "Measuring Register Shift and Investigating its Effect on Color Appearance for Different Halftoning", Proceedings of TAGA-Technical Association of the Graphic Arts, pp. 235-242, 2011.

- M. Namedanian, L. G. Coppel, M. Neuman, S. Gooran, P. Edström, and P. Kolseth, "Analysis of Optical and Physical Dot Gain by Microscale Image Histogram and Modulation Transfer Functions", Journal of Imaging Science and Technology, vol. 57, pp. 20504-120504-5, 2013. 
- M. Namedanian and S. Gooran, "Optical Dot Gain Study on Different Halftone Dot Shapes", Proceedings of TAGA-Technical Association of the Graphic Arts, pp. 96-98, 2013.

- M. Namedanian, D. Nyström, P. Zitinski Elias, and S. Gooran, "Physical and Optical Dot Gain: Characterization and Relation to Dot Shape and Paper Properties", Accepted to be published in IS\&T/ SPIE, Electronic Imaging Science and Technology, 2014.

\subsection{Dissertation Outline}

This dissertation is written as a monograph in order to provide the opportunity of presenting the ideas and the work without the restriction imposed by the publications in terms of templates and page limitations, and to avoid the considerable overlap existing in separate papers. The rest of the dissertation is organized as follows.

In Chapter 2, a brief theoretical background of concepts and methods used in the dissertation is reviewed. The chapter presents an overview of color science, including a brief introduction to color observation, CIE color spaces and color printing.

Chapter 3 provides a brief introduction to halftone color reproduction, introducing the concepts of digital halftoning and dot gain, as well as an overview of models predicting the outcome of halftone prints.

Chapter 4 gives all the technical information about the spectral measurements and the image acquisition system used for acquiring gray scale, color and multi-channel images. The test targets and the types of paper used for the studies in this dissertation have been thoroughly presented in this chapter.

Chapter 5 focuses on characterization of halftone print in the microscopic level. The histograms of the reflected and transmitted microscale images are compared. Three methods based on Murray-Davies model to estimate the total dot gain are presented. A novel approach based on microscale image histogram is proposed to separate the physical and optical dot gain.

Chapter 6 provides the validation of the proposed approach in Chapter 5 . To validate the proposed approach for separating the physical and optical dot gain, the MTFs of eleven paper samples were simulated and compared with measured MTF by knife-edge method and the MTF obtained by Monte-Carlo simulation. Moreover, in this chapter, we show 
that using the transmitted images for estimating the physical dot gain is not an appropriate approach, due to the existence of a small portion of optical dot gain.

Chapter 7 includes the characterization of different halftoning techniques in terms of optical dot gain, ink behavior, and color gamut. All the investigations have been applied and compared for two types of paper, namely coated and uncoated papers.

Chapter 8 presents a method to separate color inks from each other. The wavelength dependency of light scattering is investigated for different color inks. The chapter also presents a new approach to measure the register shift of printing devices.

Finally, Chapter 9 provides a short summary of the work and results and gives an overview of possible extensions of the dissertation work. 


\section{Chapter 2}

\section{Color Fundamentals}

Contents

2.1 Introduction $\ldots \ldots \ldots \ldots \ldots \ldots$

2.2 Color Observation . . . . . . . . . . . 11

2.2 .1 CIE Standard Illuminant _ . . . . . . . . . . 12

2.2.2 CIE Standard Observer . . . . . . . . . . . . 13

2.2.3 CIE Color Matching Functions . . . . . . . 15

2.3 CIE Color Spaces . . . . . . . . . . . 16

2.3.1 CIEXYZ Color Space . . . . . . . . . . . . 17

2.3 .2 CIELAB Color Space . . . . . . . . . . . . 18

2.3.3 Color Difference Equations . . . . . . . . . 19

2.4 Color Printing . . . . . . . . . . . . . . 21

2.4 .1 Color Mixing . . . . . . . . . . . . . . . . . 21

2.4.2 Color Printing Methods . . . . . . . . . . 25 


\section{$2.1 \quad$ Introduction}

This chapter gives a brief description of color science, color appearance models and physical properties of light in interaction with a human observer. This chapter provides the basics of color science and color printing including definitions and terminologies for the concepts used throughout the dissertation.

Many studies have been carried out in color science. Hunt $[50,51]$ provides basic knowledge on color measurement and color reproduction, Wyszecki and Stiles [115] worked on concepts and methods in color science and Fairchild presents color appearance modeling [29].

\subsection{Color Observation}

The science of color is simply called colorimetry. It includes measuring, representing, and computing color in a way which takes into account the interaction between the physical aspects of color and the physiological aspects of human vision. Colors are observed by the light spectrum, interacting with two types of photoreceptors in the eye's retina; cones and rods. Color categories and physical specifications of color are also dependent on three interacting components: light source, object and observer.

Visible light (usually referred to as light) is electromagnetic radiation that is visible to the human eye. The visible wavelength band of the spectrum is defined by the wavelengths between $380 \mathrm{~nm}$ and $780 \mathrm{~nm}$ [51].

The color of an object depends on its spectral reflectance properties. Different wavelengths, and thus different frequencies of light are perceived by the human eye as colors. The light wavelengths, which are reflected from an object, are perceived as the color of the object. Figure 2.1 illustrates the printed yellow ink perceived by human brain. As a color observer, the human eye receives the reflected or transmitted light from an object, and the brain perceives the vision. Since different humans perceive color in different ways, subjectively, attempts have been made to "standardize" the human observer as a numerical representation of what the average person sees. 


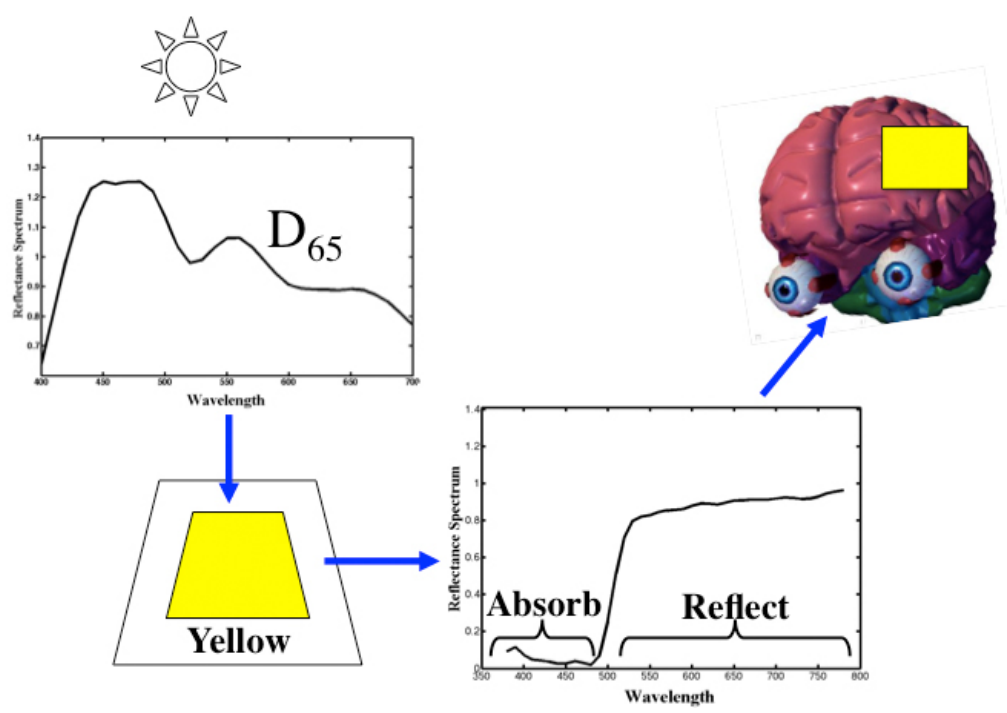

Figure 2.1: The yellow ink absorbs all the wavelengths of visible spectrum except the wavelengths between $500 \mathrm{~nm}$ to $780 \mathrm{~nm}$ and therefore in human brain it is perceived as yellow.

There is an international authority, i.e. Commission Internationale de l'Eclairage (CIE), which sets the standards for measuring, representing and computing the light, illumination, color, and color spaces.

\subsubsection{CIE Standard Illuminant}

A standard illuminant is a theoretical source of visible light that is electromagnetic radiation in the visible wavelength band at which the human eye is the most sensitive [58]. The visible wavelength band is in the range of $380 \mathrm{~nm}$ to $780 \mathrm{~nm}$, between the invisible infrared, with longer wavelengths and the invisible ultraviolet, with shorter wavelengths. Standard illuminant can be used for comparing color inks or images under different lights. The radiant flux of the observed light at each wavelength is expressed by a Spectral Power Distribution (SPD). The SPD provides the user with a visual profile of the color characteristics of a light source and describes the power per unit wavelength of an illumination.

CIE introduced the standard illuminants in 1931; Illuminants A, B, and $\mathbf{C}$ are the average incandescent light, the direct sunlight, and the average daylight, respectively. Illuminants $\mathbf{D}$ represent phases of daylight, Illuminant $\mathbf{E}$ is the equal-energy illuminant, and Illuminants $\mathbf{F}$ 
represent fluorescent lamps of various composition. In this dissertation $\mathrm{D}_{65}$ has been used as daylight of the standard illumination which is part of $\mathrm{D}$ series of illuminants. According to CIE standard definition, $\mathrm{D}_{65}$ is intended to represent average daylight and has a correlated color temperature of approximately $6500 \mathrm{~K}$. It should be noted that there are no actual $\mathrm{D}_{65}$ light sources, only simulators, but the quality of a simulator should be assessed with the CIE standard. Figure 2.2 illustrates the spectral power distribution of the illuminants $\mathrm{D}_{65}, \mathrm{~B}, \mathrm{~A}$, and Tungsten$60 \mathrm{w}$.

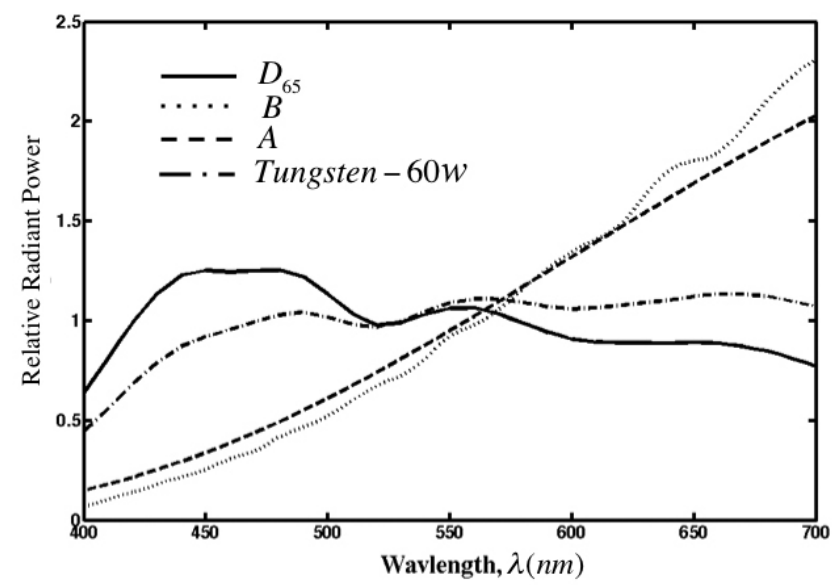

Figure 2.2: The spectral power distribution of the illuminants $D_{65}$, B, A, and Tungsten-60w.

\subsubsection{CIE Standard Observer}

In the visual observing situation, the human eye is the observer that receives the reflected light from an object and the brain perceives the vision. There are two types of photoreceptors in the human retina, rods and cones. The rods are responsible for night (scotopic) vision and the cones for daylight (photopic) vision under normal levels of illumination [40]. The cone cells are used to percept colors and they are also able to perceive finer detail and more rapid changes in images [59]. The cones are classified to the three types of pigment namely: L-cones, Mcones, and S-cones, see Figure 2.3. Hence, they are most sensitive to visible wavelengths of light that correspond to red (long wavelength, 


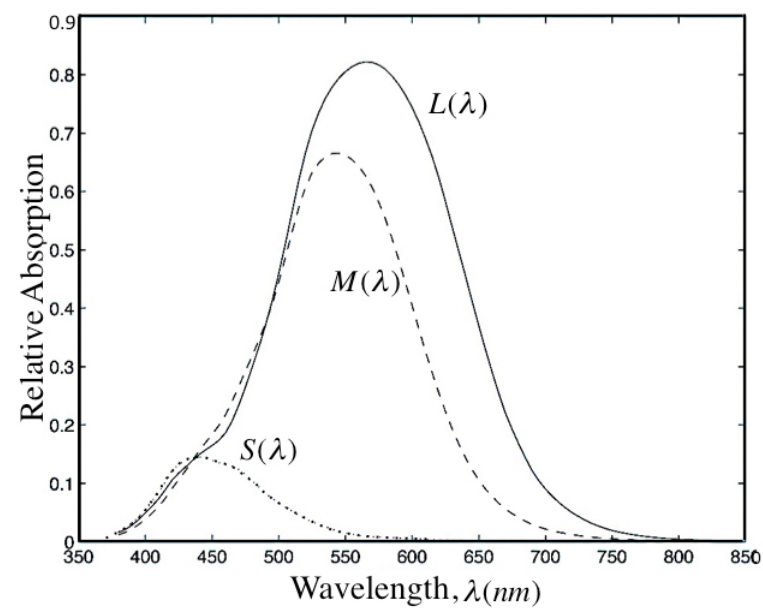

Figure 2.3: Energy absorption spectra for L, M, and S cones.

560-580 nm), green (medium wavelength, 530-540 nm), or blue (short wavelength, 420-440 $\mathrm{nm}$ ) light [102]. The stimulus from the incoming light for each type of cones is given by:

$$
\begin{aligned}
L_{t o t} & =\int_{\lambda} E(\lambda) L(\lambda) d \lambda, \\
M_{t o t} & =\int_{\lambda} E(\lambda) M(\lambda) d \lambda, \\
S_{t o t} & =\int_{\lambda} E(\lambda) S(\lambda) d \lambda,
\end{aligned}
$$

where $L(\lambda), M(\lambda)$, and $S(\lambda)$ are the spectral sensitivity functions of the cones, and $E(\lambda)$ is the incoming light's spectral photon distribution. The values $L_{t o t}, M_{t o t}$, and $S_{t o t}$, resulted from calculating such integrals over the incoming light and sensitivity functions, are referred to as tristimulus values, and describe the perceived color.

According to the human eye's receptors, all colors are reduced to three tristimulus values. Therefore, there might be different combinations of light reflected from two objects, which produce an equivalent receptor response and the same tristimulus values or color sensation. This phenomenon is called metamerism, which means that two colors that match under a given illuminant may differ when viewed under different illumination. 
Since different people perceive color in different ways, an effort has been made to standardize the human observer as a numerical representation of what the average person sees. In 1931 Wright and Guild [58], performed experiments using volunteers to assess their color vision and then developed an average. They published the $2^{\circ}$ CIE standard observer function. They called it $2^{\circ}$ because volunteers judged colors while looking through a hole that allowed them a $2^{\circ}$ field of view. In 1960, it was realized that cones are located in a larger area of the fovea. Therefore, in 1964 the $10^{\circ}$ standard observer was developed as the best representation of the average spectral response of a human observer, see Figure 2.4. However the $2^{o}$ standard observer is still used for measuring objects that are viewed at a distance.

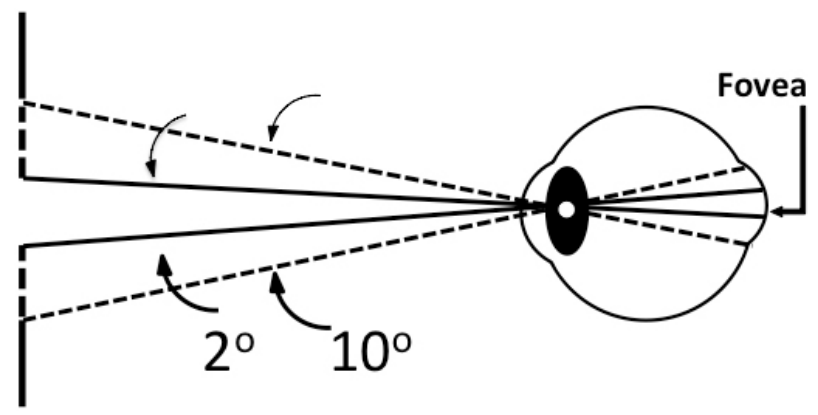

Figure 2.4: The scheme of field of view for standard observer $2^{\circ}$ and $10^{\circ}$.

\subsubsection{CIE Color Matching Functions}

The CIE system defines the specification of color matches for standard observer using color matching functions. In 1931, the CIE standard system found that $\mathrm{L}, \mathrm{M}$, and $\mathrm{S}$ are not the best representation of the sensitivity functions of human visual system's cone responses. They proposed three other well defined sensitivity functions, called $r(\lambda), g(\lambda)$, and $b(\lambda)$, found by experiment. In the experiment, the wavelengths of the red, green, and blue lights were defined: $700 \mathrm{~nm}$ for red, $546.1 \mathrm{~nm}$ for green, and $435.8 \mathrm{~nm}$ for blue [43]. Figure 2.5 (a) shows the color matching functions for red, green and blue. As it can be seen in Figure 2.5 (a) there exist negative values for $r(\lambda)$. Of course, there is no such thing as negative light. This is only a result from the fact that the 
chosen basis colors could not reproduce all colors. In order to eliminate the negative values in the color matching functions, the CIE transformed $r(\lambda), g(\lambda)$, and $b(\lambda)$ to a set of imaginary primaries, $\bar{x}(\lambda), \bar{y}(\lambda)$, and $\bar{z}(\lambda)$, using the linear transformation matrix, Equation (2.4). Figure 2.5 (b) shows the primary transformed functions. The color matching functions defined in Equation (2.4), is used to calculate tristimulus values in XYZ color space. In the following section the CIE color spaces are presented.

$$
\left[\begin{array}{c}
\bar{x}(\lambda) \\
\bar{y}(\lambda) \\
\bar{z}(\lambda)
\end{array}\right]=\left[\begin{array}{ccc}
0.49 & 0.31 & 0.20 \\
0.17697 & 0.81240 & 0.01063 \\
0 & 0.01 & 0.99
\end{array}\right]\left[\begin{array}{l}
r(\lambda) \\
g(\lambda) \\
b(\lambda)
\end{array}\right]
$$

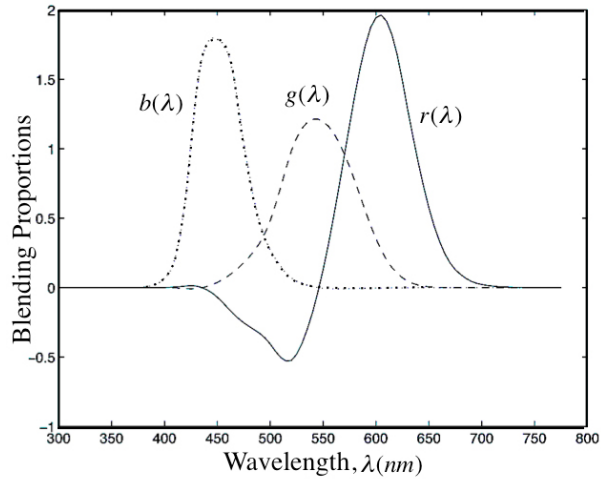

(a)

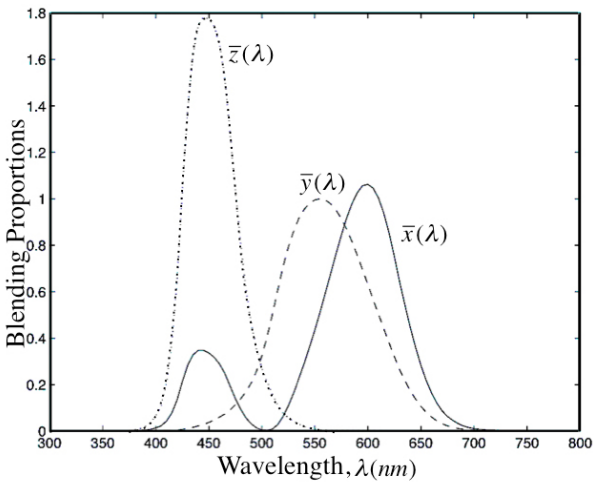

(b)

Figure 2.5: (a) The rgb color matching functions. (b) The $\bar{x} \bar{y} \bar{z}$ color matching functions.

\subsection{CIE Color Spaces}

A color space is a mathematical representation of a set of colors. A color space is useful for characterizing the color capabilities of a specific device. A color space is like a painter's palette where he creates new colors by mixing the original ones, but more precisely organized and quantified. Depending on the type of space, color space usually represents some aspects of color, such as brightness, hue or saturation. Color spaces have many different types which are suitable for different applications. A number of CIE color spaces is given as follows. 
- CIE 1931 XYZ - defining the color matching properties of the CIE 1931 standard colorimetric observer.

- CIE $1976\left(L^{*} a^{*} b^{*}\right.$ or $\left.L^{*} u^{*} v^{*}\right)$ - device independent color spaces and CIELAB space is approximately uniform.

- Chromaticity spaces, such as CIE xyY-separate the three dimensions of color into one luminance dimension and a pair of chromaticity dimensions.

- $R G B, C M Y$, and $C M Y K$ - simple device dependent spaces, express color relative to other reference spaces that can be used to reproduce color on computer, monitor, or on paper.

- HSV, HSL, and related color spaces - color spaces based on RGB, designed to be intuitive for human use.

In this chapter, we briefly describe the CIEXYZ and CIELAB color spaces, which will be later used in this dissertation.

\subsubsection{CIEXYZ Color Space}

In color science, one of the first mathematically defined color spaces is the CIE $1931 \mathrm{XYZ}$ color space $[17,104]$. The $X, Y$, and $Z$ tristimulus values can be calculated from the color matching functions (Equation (2.4)) and they are given by:

$$
\begin{aligned}
& X=k \int_{\lambda} I(\lambda) R(\lambda) \bar{x}(\lambda) d \lambda, \\
& Y=k \int_{\lambda} I(\lambda) R(\lambda) \bar{y}(\lambda) d \lambda, \\
& Z=k \int_{\lambda} I(\lambda) R(\lambda) \bar{z}(\lambda) d \lambda,
\end{aligned}
$$

where $\bar{x}, \bar{y}$, and $\bar{z}$ are the CIEXYZ color matching functions. $I(\lambda)$ is the photon distribution of the light source illuminating the object, and $R(\lambda)$, the reflectance function, is the object's influence on the incoming light. The normalization factor, $k$, is chosen to give $\mathrm{Y}=100$ for a chosen 
reference white (a perfect diffuse reflector), with spectral reflectance equal to unity for all wavelengths, i.e:

$$
k=\frac{100}{\int_{\lambda} I(\lambda) \bar{y}(\lambda) d \lambda}
$$

From the XYZ tristimulus values defined in this section, other color spaces such as CIELAB can be derived.

\subsubsection{CIELAB Color Space}

The CIELAB color space is derived from XYZ coordinates. It is an approximately uniform color space. The CIELAB space is used on any object whose color may be measured and therefore the color value can be easily compared. It was used extensively in many industries such as the textile industry to give an accurate definition to describe colors. Now it serves as one of the most well known device independent color spaces for all kinds of application. The nonlinear transformation between XYZ and CIELAB values is defined by:

$$
\begin{gathered}
L^{*}=\left\{\begin{array}{cc}
116 \cdot\left(\frac{Y}{Y_{n}}\right)^{1 / 3}-16, & \left(\frac{Y}{Y_{n}}\right)>0.008856 \\
903.3 \cdot\left(\frac{Y}{Y_{n}}\right), & \left(\frac{Y}{Y_{n}}\right) \leq 0.008856
\end{array}\right. \\
a^{*}=500 \cdot\left(f\left(\frac{X}{X_{n}}\right)-f\left(\frac{Y}{Y_{n}}\right)\right) \\
b^{*}=200 \cdot\left(f\left(\frac{Y}{Y_{n}}\right)-f\left(\frac{Z}{Z_{n}}\right)\right) \\
f(x)=\left\{\begin{array}{cc}
x^{1 / 3}, & x>0.008856 \\
7.787 x+\frac{16}{116}, & \left(\frac{Y}{Y_{n}}\right) \leq 0.008856
\end{array}\right.
\end{gathered}
$$

where the constant $X_{n}, Y_{n}$, and $Z_{n}$ are the $\mathrm{XYZ}$ values for the chosen reference white point. In the CIELAB space, $L^{*}$ axis indicates the lightness. As it can be seen in Figure 2.6 the maximum for $L^{*}$ is 100 which represents the reference white and the minimum for $L^{*}$ is 0 which represents black. Positive $a^{*}$ is corresponding to the redness and negative $a^{*}$ is green. Positive $b^{*}$ is yellow and negative $b^{*}$ is blue. 


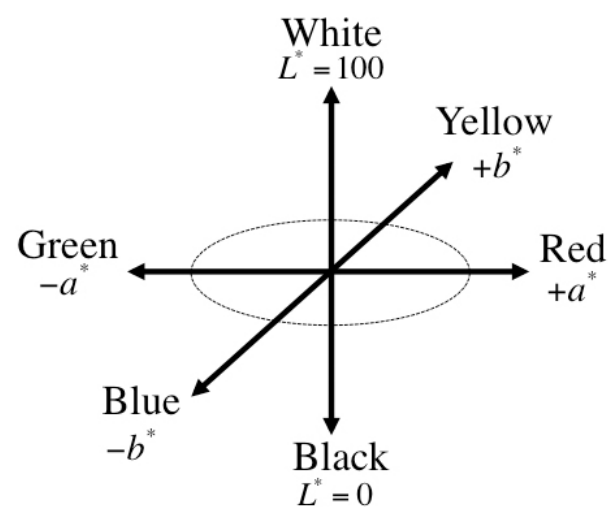

Figure 2.6: Interpretation of the $L^{*}, a^{*}$, and $b^{*}$ in CIELAB color space.

\subsubsection{Color Difference Equations}

The difference or distance between two colors is a measure for comparing colors in terms of color appearance. The CIELAB color difference equations are extensively used for quality control in industry. The CIELAB is not truly visually uniform, hence for equal perceptual color differences, the values of CIELAB color differences can vary by an order of magnitude [68]. Instead of defining a new color space, the color science community has proposed some other methods to calculate the color difference based on higher order mathematics. This was resulted in color difference equations that better correlate with visually perceived differences. In this dissertation we are going to briefly describe $\Delta E_{a b}$ and $\Delta E_{94}$.

- $\operatorname{CIE} 1976\left(\Delta E_{a b}\right)$

The $\Delta E_{a b}$ corresponding to the Euclidean distance in CIELAB color space is given by:

$$
\Delta E_{a b}=\sqrt{(\Delta L)^{2}+(\Delta a)^{2}+(\Delta b)^{2}}
$$

where $\Delta L, \Delta a$ and $\Delta b$ are the differences in $L, a$, and $b$ between the two samples, respectively. An alternative equation is expressed in terms of lightness difference, $\Delta L$, chroma difference, $\Delta C_{a b}$, and hue difference $\Delta H_{a b}$, as shown in Equation (2.14).

$$
\Delta E_{a b}=\sqrt{(\Delta L)^{2}+\left(\Delta C_{a b}\right)^{2}+\left(\Delta H_{a b}\right)^{2}}
$$


The interpretation of $\Delta E_{a b}$ colour differences is not straightforward. In some literature $\Delta E_{a b}=1$ is defined as a just noticeable difference (JND), [50,68]. Table 2.1 shows how to interpret the $\Delta E_{a b}$ between two colors shown side by side, [40].

Table 2.1: How to interpret the color difference.

\begin{tabular}{|l|l|}
\hline \multicolumn{1}{|c|}{$\Delta E_{a b}$} & Effect \\
\hline$\Delta E_{a b}<3$ & Hardly perceptible \\
\hline $3<\Delta E_{a b}<6$ & Perceptible, but acceptable \\
\hline$\Delta E_{a b}>6$ & Not acceptable \\
\hline
\end{tabular}

- $C I E 1994\left(\Delta E_{94}\right)$

The color difference, $\Delta E_{94}$, is calculated as a weighted meansquare sum of the differences in lightness, $\Delta L^{*}$, chroma, $\Delta C^{*}$, and hue, $\Delta H^{*}$. The CIE94 color difference, $\Delta E_{94}$ is given by:

$$
\Delta E_{94}=\sqrt{\left(\frac{\Delta L}{k_{L} S_{L}}\right)^{2}+\left(\frac{\Delta C_{a b}}{k_{C} S_{C}}\right)^{2}+\left(\frac{\Delta H_{a b}}{k_{H} S_{H}}\right)^{2}},
$$

where

$$
\begin{gathered}
S_{L}=1, \\
S_{C}=1+K_{1} C_{1}, \\
S_{H}=1+K_{2} C_{1},
\end{gathered}
$$

where the weighting functions $S_{L}, S_{C}$, and $S_{H}$ vary with the chroma of the reference sample, and $K_{1}$ and $K_{2}$ depend on the application as follows,

\begin{tabular}{c|c|c} 
& graphic art & textiles \\
\hline $\mathrm{K}_{1}$ & 0.045 & 0.048 \\
$\mathrm{~K}_{2}$ & 0.015 & 0.014
\end{tabular}

The variables $K_{L}, K_{C}$, and $K_{H}$ are called parametric factors and are included in the equation for allowing adjustments to be made independently to each color difference term. The adjustment is needed to clarify any deviations from the reference viewing conditions. This is caused by the component specific variations in the 
visual tolerances [39]. Under the reference conditions defined by CIE 1994 color-difference model [69], they are set to,

$$
K_{L}=K_{C}=K_{H}=1
$$

For the neutral colors (black, white, gray, and sometimes brown and beige) and under reference conditions, $\Delta E_{94}$ is equal to $\Delta E_{a b}$, while for more saturated colors $\Delta E_{94}$ is smaller than $\Delta E_{a b}[40]$.

\subsection{Color Printing}

\subsubsection{Color Mixing}

Under optimal viewing conditions, the human eye can approximately recognize more than 10 million different colors [114]. All the colors are created by combining different ratios of minimum three primary colors. There are two basic systems of mixing colors. One system of color mixing takes place when two or more colored light sources are combined and the other one takes place by mixing colorants such as ink, dyes and paint.

\section{- Additive Color Mixing}

Color mixing with colored lights is called additive color mixing. Computer monitors and televisions are two applications of additive color mixing. The additive primary colors are red, green and blue. Combining equal amount of two of these additive primary colors results in the additive secondary colors cyan, magenta and yellow as follows,

$$
\begin{aligned}
\text { Red }+ \text { Green } & =\text { Yellow } \\
\text { Red }+ \text { Blue } & =\text { Magenta } \\
\text { Green }+ \text { Blue } & =\text { Cyan } \\
\text { Red }+ \text { Green }+ \text { Blue } & =\text { White }
\end{aligned}
$$

Combining all three additive primary colors in equal amounts produces the gray color (in case of full intensity, it would be white) and the absence of all three colors results in black. It should be noticed that combining two or more additive colors creates a lighter color that is closer to white. A conceptual model to illustrate all the primary additive colors and their combinations in the visible spectrum is the RGB color cube, see Figure 2.7. Each of the eight 
vertices on the color cube shows a single primary color plus black and white. Any point inside or on the surface of the color cube is an additive mixture of the primary colors.

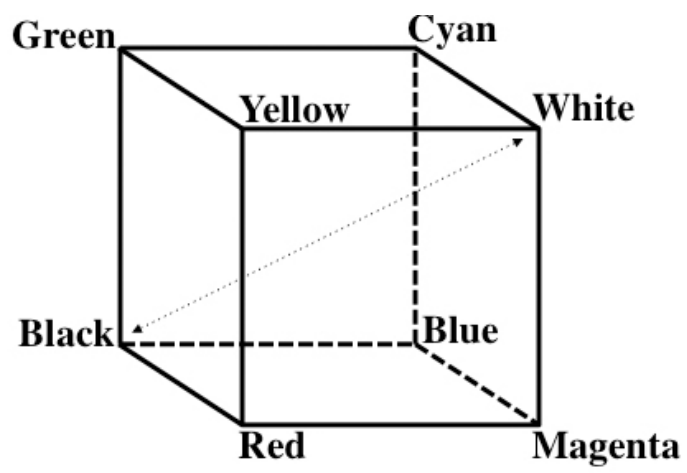

Figure 2.7: The RGB color cube shows the additive primary colors and their combinations.

- Subtractive Color Mixing

Color mixing with a set of dyes, inks, and paint pigments to create a wider range of colors is called subtractive color mixing. The printed ink on a substrate acts as a filter in the visible wavelength band. The ink can absorb some part of the light in some wavelengths and reflect back the rest of light from the paper. The subtractive color mixing occurs when the light is filtered through the printed ink. For example, a magenta ink appears magenta because it absorbs all wavelengths of the light except the wavelengths we call magenta. The subtractive primary colors are cyan, magenta and yellow. By combining a same amount of two primary subtractive colors the secondary colors of red, green, and blue and by combining all three primary subtractive colors the black color is produced as follows,

$$
\begin{aligned}
\text { Magenta+Yellow } & =\text { Red } \\
\text { Cyan+Yellow } & =\text { Green } \\
\text { Cyan+Magenta } & =\text { Blue } \\
\text { Cyan+Magenta+Yellow } & =\text { Black }
\end{aligned}
$$


We consider the printed paper as being made of two layers, the full tone ink, and the substrate layer. Incident light $\mathrm{I}(\lambda)$ on the printed paper goes through the ink layer with the spectral transmittance of $T(\lambda)$, and reflects back in the substrate layer and goes through the ink layer again, see Figure 2.8 (a). Regardless of the light scattering effect of the paper, the effective final spectral radiance is produced as follows,

$$
I_{t o t}(\lambda)=T^{2}(\lambda) \cdot I(\lambda) \cdot R_{p}(\lambda)
$$

where $R_{p}(\lambda)$ is the reflectance of paper. For the two full tone color inks printed on top of each other in Figure 2.8 (b), the term multiplicative color mixing, rather than subtractive, would be more mathematically correct.

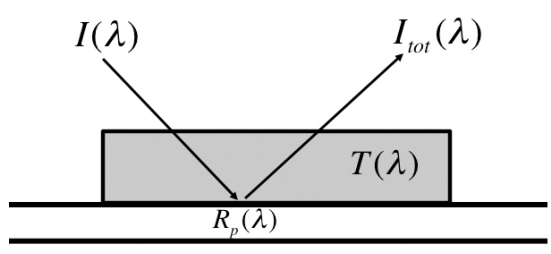

(a)

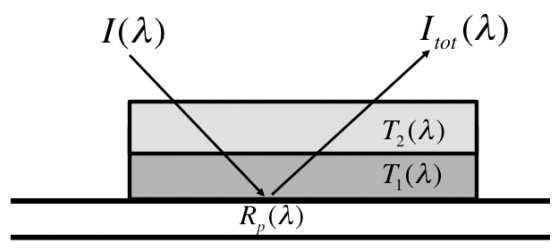

(b)

Figure 2.8: (a) One full tone ink layer printed on the paper.

(b) Two full tone ink layers printed on the paper.

Equation (2.19) is used to express the effective spectral radiance, when two full tone color inks are printed on top of each other,

$$
I_{\text {tot }}(\lambda)=T_{1}^{2}(\lambda) \cdot T_{2}^{2}(\lambda) \cdot I(\lambda) \cdot R_{p}(\lambda)
$$

where $T_{1}(\lambda)$ and $T_{2}(\lambda)$ are the transmittance functions of the two color inks. Ideally, each primary color absorbs one-third of the visible spectrum and transmits two-thirds [81]. Figure 2.9 (a) shows the ideal spectral characteristics of these three primary colors. Here it should be noted that these ideal primary color inks do not exist. Figure 2.9 (b) shows a measured set of spectral characteristics for primary and secondary color inks for an offset print press. Ideally cyan, magenta, and yellow are sufficient to produce 
a wide range of colors, e.g. equal amounts of these three primary colors at full tone should produce black, but in practice, a dark brown color is produced instead. Hence a fourth real black ink is added to obtain more accurate colors and avoid printing the three primary inks on top of each other. This is called the CMYK color system [67].
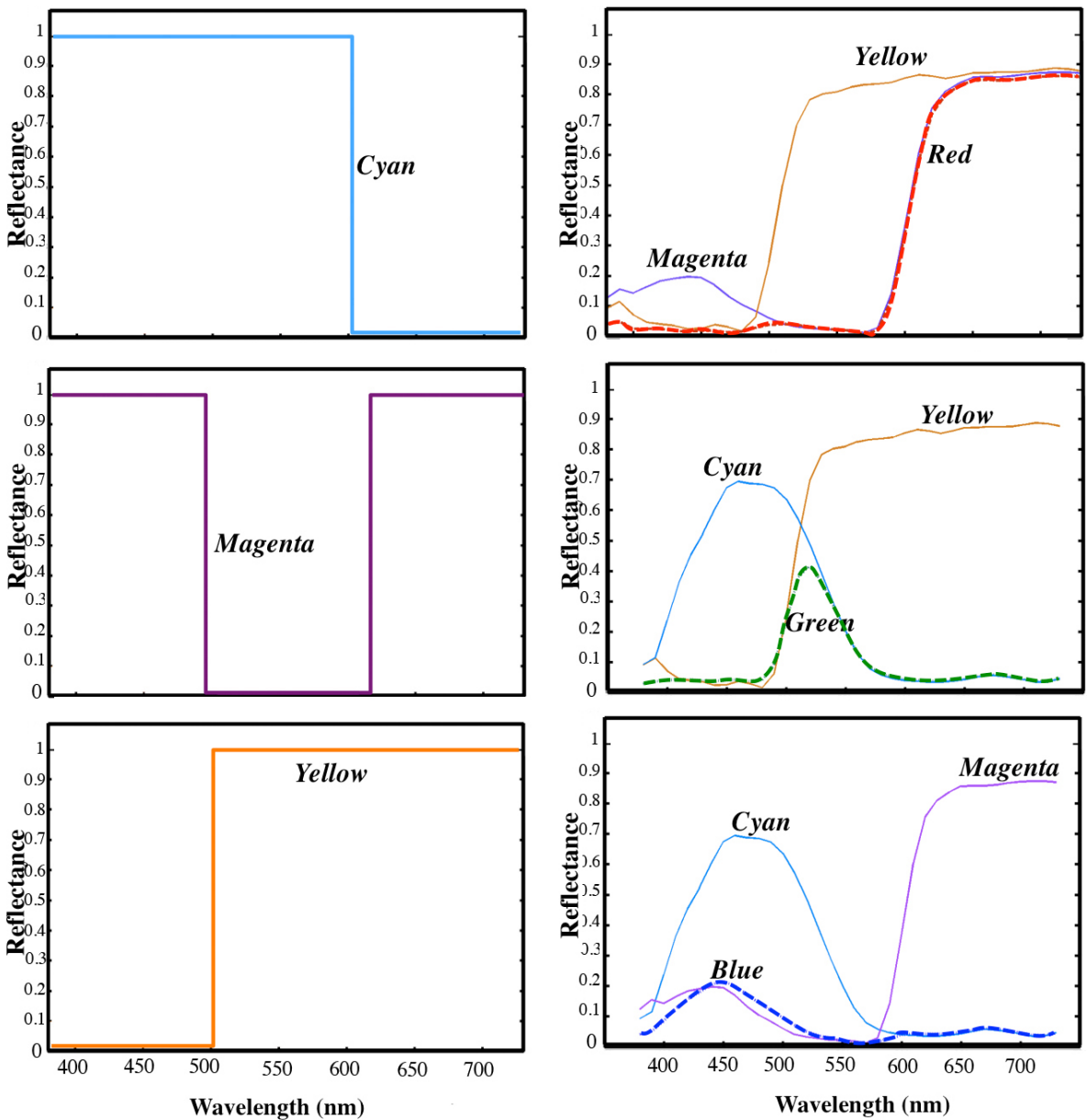

(a)

(b)

Figure 2.9: (a) Spectral characteristics of three ideal primary subtractive colors. (b) Spectral characteristics of measured primary and secondary subtractive colors. 


\subsubsection{Color Printing Methods}

Printing devices can be classified based on the printing techniques. In terms of the technology used, a number of main types of printing techniques are categorized as follows.

- Offset lithography: is one of the most common flat printing techniques, wherein ink is transferred from a plate to a rubber blanket, then to the printing surface.

- Digital printing: refers to methods of printing from a digital based image directly to a variety of media. The most popular methods include inkjet or laser printers that deposit pigment or toner onto a wide variety of substrates including paper, photo paper, canvas, glass, metal, marble.

- Flexography: is a form of printing process which uses a flexible relief plate. Flexography is used for packaging products that include cardboard boxes, grocery bags, gift wrap, and bottle labels.

In this section, the offset printing technique, which is used in this research study, is briefly described.

The offset printing is a printing process in which the image is transferred indirectly to a substrate. Text or pictures are imaged onto printing plates such as metal, polyester, and paper. The best plate material is aluminum, which is more costly, but provides a high-quality offset printing. Each of the primary colors, cyan, magenta, yellow, and black have a separate plate. The image is transferred to a rubber blanket, and from the rubber blanket onto the paper, Figure 2.10. This process is called "offset" because the plate never directly touches the paper.

The quality of image, printed by the offset printing process is dependent on a variety of parameters, such as ink, inking system, blanket, plate making, cylinder pressure, dampening system, temperature and substrate. Most of the parameters usually change slowly over a long period of time such as days or months. To compensate for variations of the parameters, the operator has to constantly monitor the print and take appropriate actions during the print run.

The cylinder tension causes the softer paper surface to be more compressed and deformed. In this case for the uncoated paper the ink 
spreads more through the pores of the paper and makes an un-uniformed dot shape [13].

In a multicolor offset press, the transportation of the printing substrate from one printing unit to the other one must be very precise. An imprecise transportation of the printing substrate causes register variation. Register variation is a displacement of the printing detail from sheet to sheet. Due to the dampening in wet printing (i.e. offset printing) register variation can cause visible color shifts. Reducing register variation in a press is one of the biggest tasks and challenges for every print machine manufacturer. However a zero tolerance of register variation of printing substrate is impossible due to technical circumstances (i.e. high printing speed and instability of proportions of substrate within the printing process) [41]. In Section 8.5, we present an approach to measure the register shift based on microscale images.

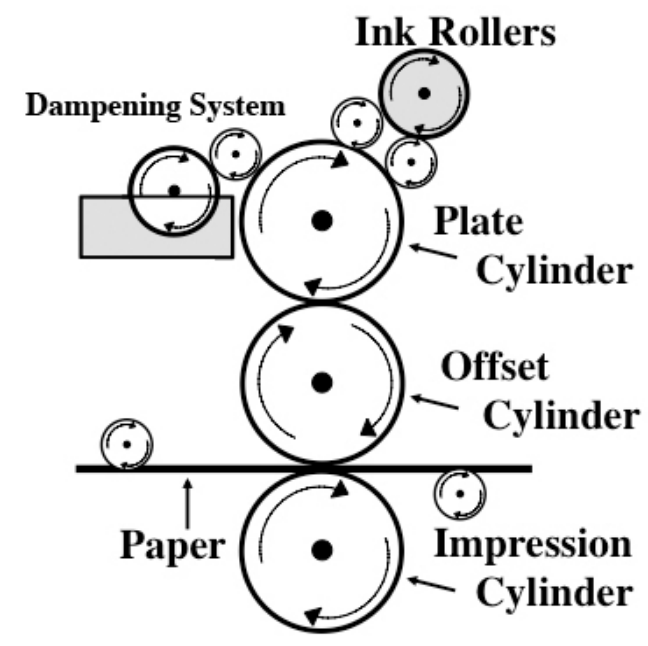

Figure 2.10: The basic scheme of offset print press. 


\section{Chapter 3}

\section{Halftone Color Reproduction}

\section{Contents}

3.1 Introduction $\ldots \ldots \ldots \ldots \ldots$

3.2 Digital Halftoning . . . . . . . . . . . 29

$3.2 .1 \quad$ AM and FM Halftoning . . . . . . . . . . 31

3.2.2 FM Second Generation Halftoning . . . . . . 32

3.2 .3 Color Halftoning . . . . . . . . . . . . . . 34

3.3 Dot Gain . . . . . . . . . . . . . 35

3.3 .1 Physical Dot Gain . . . . . . . . . . . . 36

3.3.2 Optical Dot Gain . . . . . . . . . . . . . 36

3.4 Modeling Halftone Color Reproduction . . . 38

3.4.1 Murray-Davies Model . . . . . . . . . . . . . 39

3.4 .2 Yule-Nielsen Model . . . . . . . . . . . . . . . 41

3.4.3 Expanded Murray-Davies Model . . . . . . . 43

3.4.4 Neugebauer Model . . . . . . . . . . . . . . . 43

3.4.5 Yule-Nielsen Modified Neugebauer Model . . 45

3.4.6 Clapper-Yule Model . . . . . . . . . . . . . . 46

3.4.7 Kubelka-Munk Model . . . . . . . . . . . . . 47

3.4 .8 Monte-Carlo Simulations . . . . . . . . . . 50 


\subsection{Introduction}

Most of the image reproduction devices, particularly the printing devices, are restricted to few colors, while the digital image mostly consists of millions of colors. Halftoning is one of the most important parts of the image reproduction process for devices with a limited number of colors. The printed color is affected by ink spreading and ink penetration in the substrate that makes printed dots become larger, which is referred to as the physical dot gain. Lateral light scattering in printed paper causes printed dots to appear larger than their physical size, which is called optical dot gain. The aim of this chapter is to provide a brief background to halftone color reproduction and also to describe the concepts of digital halftoning and dot gain.

To calibrate a color printer, a relationship between the input signals to the printer and the colorimetric measurements of the resulting printed colors is required. This relationship, which is called the printer characterization function, is obtained by measuring a group of printed color patches and applying some interpolation among the measurements [10]. Another approach is to predict the characterization function with a printer model. There exist many models to predict the color output of the halftone print. In this chapter an overview of some previous works on several printer models is given.

\subsection{Digital Halftoning}

Most printing devices are restricted to cyan, magenta, yellow and additional black color inks while the digital image mostly consists of millions of colors. To reproduce a continuous tone digital image, one should first transfer it into a binary image consisting of 1's and 0's, which is called bitmap. A 1 at a pixel represents an ink dot at that particular position and a 0 means that the corresponding position should remain empty or unprinted. This transformation from a continuous tone image to a binary bitmap image is referred to as Halftoning, or Screening. The printing device usually creates halftone dots by means of a halftone cell. The fractional area of the halftone cell that is covered by the ink should represent the average color of the corresponding area in the original image.

Figure 3.1 shows two $8 \times 8$ halftone cells. The small dots in each halftone cell are called micro dots. The halftone area in Figure 3.1 (a) 
is $2 \times 2$ and thus represents the gray tone of $4 / 64$. The gray tone represented in Figure 3.1 (b) is $44 / 64$. Generally it is possible to represent $n^{2}+1$ different gray tones by $n \times n$ halftone cells. Therefore in this case the halftone cells can produce $65\left(=8^{2}+1\right)$ different gray tones.

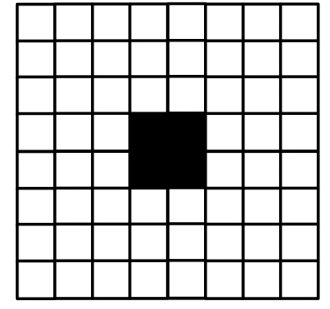

(a)

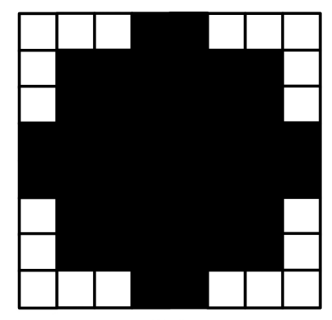

(b)

Figure 3.1: Two halftone cells. (a) The gray tone is $4 / 64$. (b) The gray tone is $44 / 64$.

The number of halftone cells per inch is called line screen ruling or screen frequency and is denoted by lpi, lines per inch. When lpi is increased, the halftone cell and consequently the halftone dot becomes smaller and therefore it is harder for a human eye to detect the halftone dots. It has been previously shown that the halftone dots are not recognized by the eye from the normal viewing distance at screen frequencies above $200 l p i[65,66]$. The number of the micro dots per inch is called the print resolution and is denoted by $d p i$, dots per inch. The ratio of the print resolution and screen frequency determines the number of represented gray levels and is given by following equation,

$$
\text { Number of gray levels }=\left(\frac{d p i}{l p i}\right)^{2}+1
$$

According to Equation (3.1), for a constant $d p i$, a higher lpi will result in a lower number of gray levels. Choosing an appropriate $l p i$ is therefore a trade-off between the number of gray tones and the fine details [32].

In order to print a color image, first it must be separated into the primary color channels that the print device utilizes. As described in Chapter 2, the primary subtractive colors cyan, magenta, yellow, and additional black color inks are most often used in color printing. Each channel should be halftoned individually by the chosen halftoning methods.

Conventionally, halftoning is accomplished either by changing the size of the dots or by changing the number of dots [42]. The halfton- 
ing methods can mainly be classified into two main categories, namely Amplitude Modulation (AM) and Frequency Modulation (FM). There is also another representative model of FM halftoning which is called FM second generation. These three types of halftoning have been used in this dissertation, and hence more explanation about these three methods follows in the following sections.

\subsubsection{AM and FM Halftoning}

In AM halftoning, the size of the halftone dots is varied depending on the gray level value of the corresponding part in the original digital image, while their spatial frequency is constant. The dot in the halftone cell becomes bigger, as the tone value gets darker and smaller when the tone value becomes lighter. On the other hand, in FM, the size of the dots is constant while the number (the frequency of micro dots) varies. Figure 3.2 shows two examples of AM and FM halftoning methods for tone values of $6.25 \%$ and $25 \%$ in the case of $8 \times 8$ halftone cells. Although the two halftone cells in each column represent the same tone value, the upper halftone cells represent AM and the lower ones represent FM halftones.

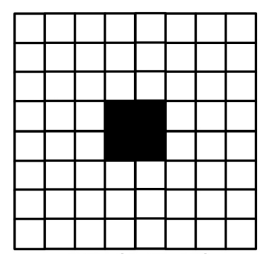

$\operatorname{AM}(6.25 \%)$

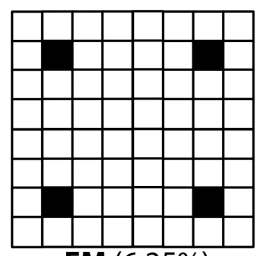

FM (6.25\%)

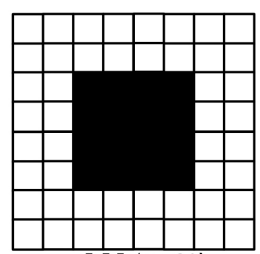

AM (25\%)

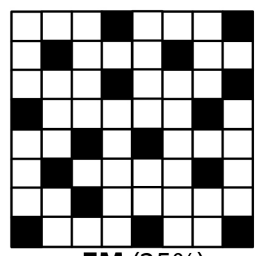

FM (25\%)

Figure 3.2: Examples of AM and FM halfoning for the gray levels $6.25 \%$ and $25 \%$ in the case of $8 \times 8$ halftone cells.

It must be mentioned that most of FM halftoning techniques (also called FM first generation in this dissertation), such as error-diffusion 
and iterative halftoning techniques, do not use any halftone cells and the micro dots are placed based on certain criteria [32].

The AM and FM halftoning have some advantages and disadvantages. The FM halftoning methods are better to reproduce the details, especially when the screen frequency is low due to the mechanical limitations of the print press. The AM halftoning methods are better to reproduce a homogenous pattern, i.e. parts of the image where the tone values change slowly. Due to the advantages and disadvantages of these methods, many researchers have used the combination of both methods, which is called Hybrid halftoning technique. The main idea behind this method is to use FM for the details of the original image and AM for the rest of the image $[12,31,33]$.

\subsubsection{FM Second Generation Halftoning}

New generation of FM is the so called FM second generation (FM2nd) which overcomes some of the disadvantages associated with the conventional (first generation) FM halftoning techniques. Unlike the first generation FM halftoning that places small dots (micro dots) of equal size, in FM2nd both the size of the dots and their frequency vary. This improves printability and reduces noise. On the other hand, unlike the conventional AM halftoning technology, FM second generation has no uniform dot shape and therefore prevents producing visible moiré patterns [106].

In this dissertation a method of FM2nd is used that has been developed at MIT (Media and Information Technology) research group of Linköping University. This method is based on the threshold halftoning method. In the threshold halftoning, depending on the content of the original image, the result will vary due to the form of the threshold matrix. This technique can simply be described by the following equation,

$$
b(i, j)=\left\{\begin{array}{lll}
1 & \text { if } \quad g(i, j) \geq t(i, j) \\
0 & \text { if } \quad g(i, j)<t(i, j)
\end{array}\right.
$$

where $b, g$ and $t$ denote the final halftoned image, the original image and the threshold matrix respectively. The pixel value at each position $(i, j)$ in $g$ is compared with the corresponding position in the threshold matrix $t$. If this is equal or bigger than the threshold, then a 1 (dot) is set at the corresponding position in the halftoned image $b$. Otherwise, a 0 (white 
dot) is set there. The methodology used to create this threshold matrix is based on a FM method described in [34]. The threshold matrix $t(i, j)$ is based on a parameter called sigma. This parameter plays a significant role in the quality control of the printed image. By changing the sigma value, one can get different halftone dot patterns. Due to a future patent submission, more detailed information regarding the method on how to obtain the threshold matrix, $t$, is not allowed to be given here.

In this dissertation, three different values for sigma have been selected and named regarding the dot size which they produce. For example, the sigmas which create the big, medium, and small size of the dots, are called FM2nd_Big, FM2nd_Medium, and FM2nd_Small, respectively. Figure 3.3 illustrates three depicted threshold matrices $t(i, j)$ created by using three different size of sigmas (big, medium, and small) in the upper row. The lower row shows the $40 \%$ gray tone level halftoned by their corresponding threshold matrices.

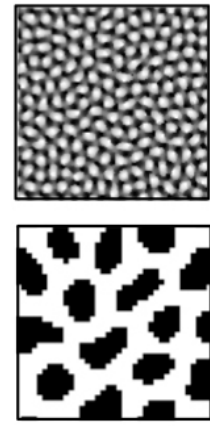

FM2nd_Big
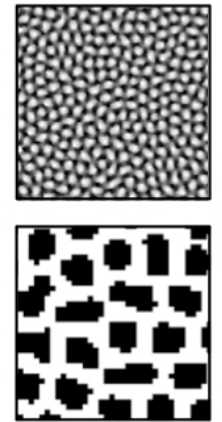

FM2nd_Medium
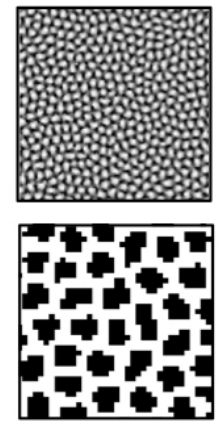

FM2nd_Small

Figure 3.3: The depicted threshold matrices $t(i, j)$ for FM2nd_Big, FM2nd_Medium, and FM2nd_Small in the upper row. The $40 \%$ gray tone value halftoned by using their corresponding threshold matrices in the lower row.

Figure 3.4 shows an enlargement of a part of a test image halftoned by AM, FM2nd_Big, FM2nd_Medium and FM_Small halftoning techniques. It is obvious that halftoning is one of the most important parts of the image reproduction process, especially in printing. The print quality is also dependent on the halftoning properties. Characterization of the halftoning method is however not so simple because there are other factors that also affect the print results. The properties of the materials such as paper and ink and the geometrical distribution of ink 
such as resolution, location, size and shape of the dots are some factors that have the most significant effect on the print quality. Characterizing the halftone print properties is useful for system calibration and quality control of the color reproduction.
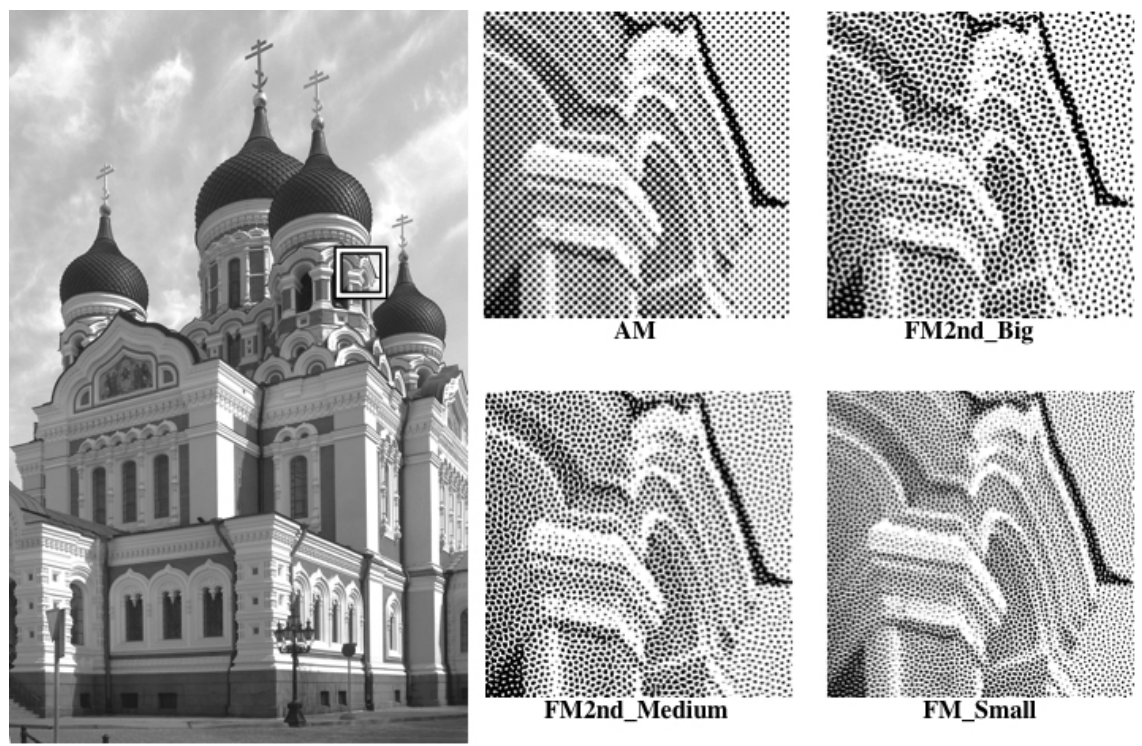

Figure 3.4: An enlarged part of an image halftoned by AM, FM2nd_Big, FM2nd_Medium, and FM_Small halftoning techniques.

\subsubsection{Color Halftoning}

Three different halftoning methods have already been introduced in this chapter. To halftone a color image, each color channel, which most commonly are cyan, magenta, yellow, and black, is halftoned by a chosen halftoning technique. In AM color halftoning all channels can be halftoned using the same screen angle assuming that there is no misregistration. However, in practice there is often some misregistration between the printed color channels. Minor misregistration of a halftone screen can cause color shift and unwanted moiré patterns. The microscale image captured by high-resolution camera in Figure 3.5(a) illustrates magenta and yellow dots printed in correct position and correct registration. Figure 3.5 (b) shows that printed cyan and magenta dots are shifted in position. 


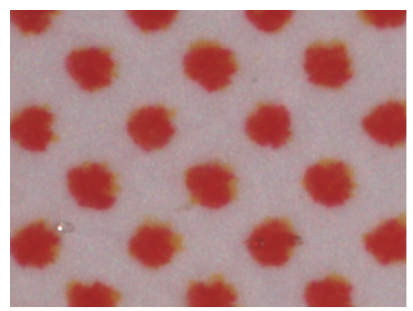

(a)

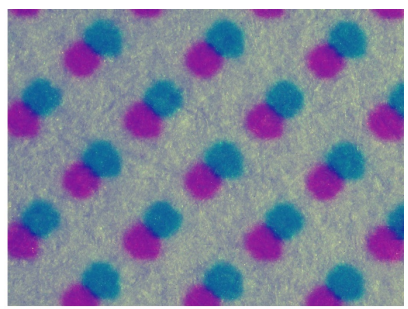

(b)

Figure 3.5: (a) Printed magenta and yellow dots in correct position.

(b) Printed cyan and magenta dots which are shifted in position.

In AM color halftoning, to reduce the effect of misregistration, four different angles are used for cyan, magenta, yellow and black. Due to the lower sensitivity of eye at $45^{\circ}$ the color with the strongest contrast, black, is halftoned and placed at this angle. The weakest color, yellow, is halftoned at $0^{\circ}$ degrees, where the human eye is most sensitive. Cyan and magenta are placed at $15^{\circ}$ and $75^{\circ}$, respectively. Figure 3.6 (a) shows the scheme of AM color halftoning with different screen angles for cyan, magenta, yellow and black channels. Using different angles for different channels reduce the effect of misregistration, but on the other hand introduces a new type of patterns, rosette patterns, which are quite visible at lower screen frequencies. Figure 3.6 (b) illustrates a type of rosette patterns that may occur in AM color halftoning. In FM halfoning of a color image, the FM techniques are applied to the color channels. Normally the color channels are halftoned independently and there is no need for rotated screen and therefore moiré patterns are generally avoided.

\subsection{Dot Gain}

The printed dots generally appear bigger than their normal size in the digital bitmap. The dots become physically bigger due to the ink spreading on the paper's surface and other distortions produced by the printer. This is what we call the physical (mechanical) dot gain. Another reason why the printed dots appear bigger than their real physical size is the diffusion of the light in the paper or substrate. This is called the optical dot gain. These concepts are briefly described in the following subsections. 


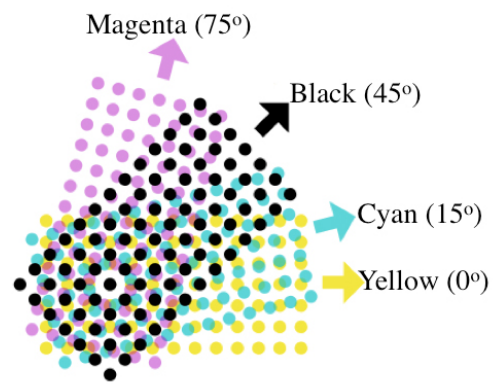

(a)

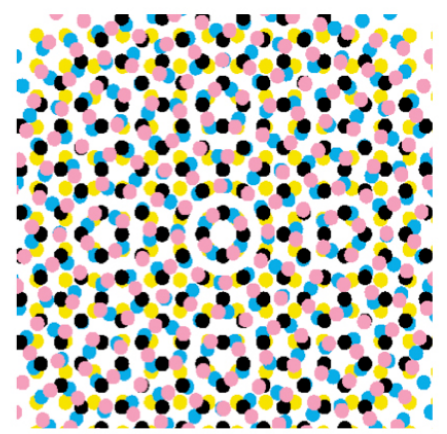

(b)

Figure 3.6: (a) Scheme of typical AM color halftoning for cyan $\left(15^{\circ}\right)$, magenta $\left(75^{\circ}\right)$, yellow $\left(0^{\circ}\right)$, and black $\left(45^{\circ}\right)$. (b) A type of rosette pattern that may occur in AM color halftoning

\subsubsection{Physical Dot Gain}

Due to many different factors the printers or the print presses are not able to print the dots exactly the same size and shape as their correspondence in the bitmap. Mostly, the dots are printed bigger, which makes the printed image darker. Physical dot gain is caused by ink spreading around halftone dots. Several factors can contribute to the increase in halftone dot area. Different paper types have different ink absorption rates; for example uncoated papers absorb more ink than coated ones. Printing pressure can squeeze the ink out of its dot shape causing gain. Ink viscosity is a contributing factor with coated papers; higher viscosity inks can resist the pressure better. The pressure from the printing cylinder also plays a significant role; the bigger the pressure the bigger the physical dot gain $[3,107]$.

Figure 3.7 shows a microscale image of a printed dot halfoned by FM2nd halftoning technique. Due to the factors explained above the ink spread around the digital dot, and hence the printed dot becomes bigger than the digital dot in the bitmap.

\subsubsection{Optical Dot Gain}

Photon migrations within the paper from non inked to inked regions tend to increase the photon absorption and thus decrease the halftone reflectance. In this case the dots appear effectively larger than their 


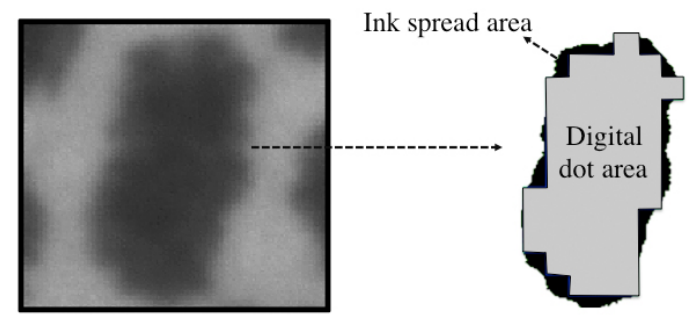

Figure 3.7: The microscale printed dot halftoned by FM2nd technique. The ink spreads on the papers's surface, and hence the printed dot is bigger than the digital dot in the bitmap.

physical size (also called Yule-Nielsen effect), that makes an accurate color prediction very difficult $[47,98,117]$. Figure 3.8 shows a simple illustration of five possible paths that a photon can travel when it enters a halftone print on paper. Photon $\mathrm{A}$ is reflected through the ink, photon $\mathrm{B}$ is absorbed in the ink layer, photon $\mathrm{C}$ is reflected from the paper's surface, and photon $\mathrm{D}$ is scattered inside the paper and reflected from the paper's surface. Photons E and F are the reasons for the optical dot gain phenomenon. Photon E enters the unprinted paper and scatters inside the paper and is partially filtered by the ink layer on its way back. Photon $\mathrm{F}$ enters the ink layer and gets partially absorbed by the ink layer, and then scatters inside the paper and finally exits from the unprinted area [88].

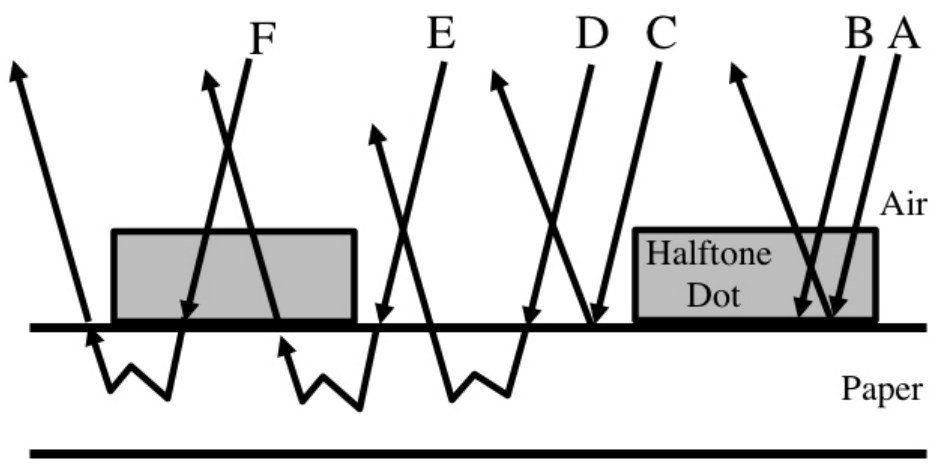

Figure 3.8: Different paths for a photon entering a halftone print. Path E and F illustrates the reason for optical dot gain.

Previously in Section 2.4 a model of light transfer behavior into the halftone print has been described, regardless of the light scattering effect 
of the paper. Due to the light scattering effect of the paper, the incident light which goes through the ink layer, is scattered in the substrate layer and goes back again through the ink layer $[28,54,101]$.

The light scattering property can be expressed by Point Spread Function (PSF). The probability for a photon to enter the surface of the dots and to be scattered in the paper and exit from unprinted parts of the paper can be described by PSF [38]. Since PSF is closely related to the optical properties of the paper it can be used to characterize the light scattering effect of different papers. Therefore, Equation (2.18) is extended to Equation (3.3) as follows,

$$
o(x, y)=I\left\{T(x, y) * \operatorname{PSF}_{\mathrm{p}}(x, y)\right\} \cdot T(x, y) R_{\mathrm{p}}
$$

where $o(x, y)$ is the spatial distribution of intensity of reflected light from the halftone print, $I$ is the intensity of incident light, $T(x, y)$ is the spatial distribution of ink layer transmittance, $\operatorname{PSF}_{\mathrm{p}}(x, y)$ is the PSF of the paper, and $R_{\mathrm{p}}$ is the reflectance of the paper. The sign (*) denotes convolution and $(\cdot)$ denotes element wise multiplication. In Equation (3.3), both I and $R_{\mathrm{p}}$ are wavelength dependent, however for simplicity and without loss of generality, the parameter $(\lambda)$ is omitted from the formulation. The spatial distribution of reflectance from the halftone print $R(x, y)$ can be described by the ratio between the intensities of the reflected light and the incoming light by,

$$
\begin{aligned}
& R(x, y)=\frac{o(x, y)}{I} \\
= & \left\{T(x, y) * \operatorname{PSF}_{\mathrm{p}}(x, y)\right\} \cdot T(x, y) R_{\mathrm{p}}
\end{aligned}
$$

The reflectance $R(x, y)$ is affected by both physical and optical dot gain, while $T(x, y)$ is only affected by the physical dot gain. In Chapter 5 , a new method is proposed to separate the physical dot area from the paper. When the physical dot area $T(x, y)$ is separated, it is possible to estimate the PSF which is related to optical properties of the paper. In Chapter 6, an MTF model is presented and compared with the existing methods, to simulate the light scattering effect of the paper.

\subsection{Modeling Halftone Color Reproduction}

Calibrating the color print devices, requires to find a relation between the input color and the measurement results of the printed colors. The 
relation between color measurements and amounts of cyan, magenta, yellow inks is highly non-linear and depends on many different parameters: spectral reflectance of the inks, paper properties, halftoning techniques, ink spreading, light scattering, etc. This relationship is usually obtained by printing and measuring a large number of color patches and applying some interpolation among the measurements [10].

Another approach is to predict the color output. Many models have been previously proposed to predict the color output of halftone prints $[2,73,82,120]$. In this section some well-known models that have been used in many contexts are presented. The models describe the prediction of color output in terms of spectral reflectance or tristimulus values.

\subsubsection{Murray-Davies Model}

One of the most well-known and simple models to predict the reflectance of a halftone print is the Murray-Davies model, Equation (3.5), [73].

$$
R(\lambda)=a R_{i}(\lambda)+(1-a) R_{p}(\lambda)
$$

where $R(\lambda)$ is the predicted reflectance of the halftone print, $a$ is the fractional dot area of the ink, $R_{i}(\lambda)$ is the reflectance spectrum of the ink at full coverage, and $R_{p}(\lambda)$ is the reflectance spectrum of the paper. The $(\lambda)$ indicates that all three reflectance values are a function of wavelength. Note that in this model the fractional dot area $a$ is supposed to be the physical dot coverage after print, excluding the optical dot gain. However this model is often used to approximate the effective dot area after print including physical and optical dot gains. This is due to the fact that the measured reflectance spectrum includes the effect of optical dot gain. Figure 3.9 shows the predicted spectral reflectance for a $90 \%$ cyan halftoned patch that is consistently higher than the measured reflectance. This effect is called dot gain, the phenomenon whereby measured prints are always darker than the predicted ones. This dot gain includes both physical and optical dot gain.

By using the Murray-Davies model the effective dot area $a_{e f f, R}\left(a_{r e f}\right)$ can be estimated by minimizing the root mean square difference $\Delta$ RMS between calculated (Equation (3.5)) and measured reflectance spectra. After the effective dot area, $a_{e f f, R}\left(a_{r e f}\right)$ which founds the calculated reflectance spectrum can then be given as follows,

$$
R_{C a l c}(\lambda)=a_{e f f, R}\left(a_{r e f}\right) R_{i}(\lambda)+\left(1-a_{e f f, R}\left(a_{r e f}\right)\right) R_{p}(\lambda)
$$




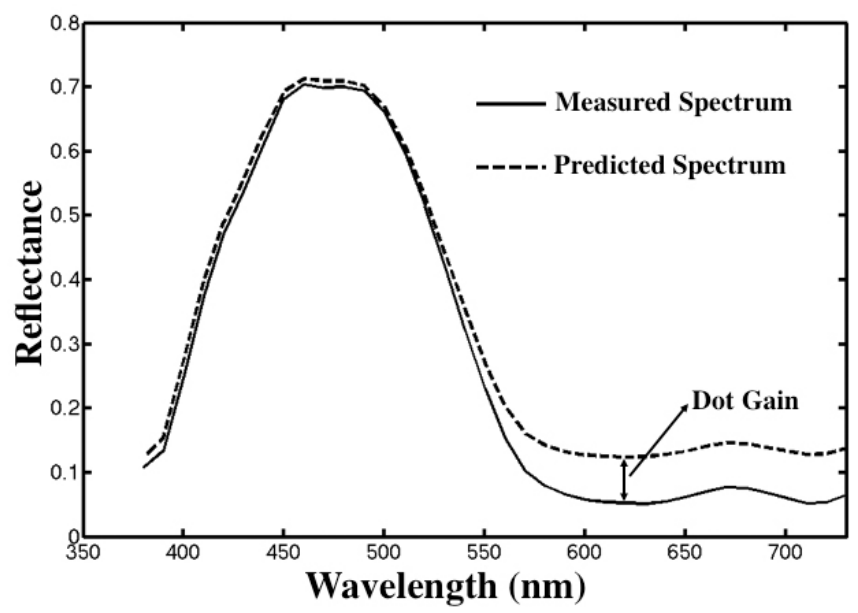

Figure 3.9: Predicted and measured spectral reflectance for $90 \%$ cyan halftoned patch. Predicted data is made using the MurrayDavies model in Equation (3.5).

where $a_{r e f}$ and $a_{e f f, R}\left(a_{r e f}\right)$ are the reference area and the effective dot area after print, respectively. The $R$ subscripts indicate that the estimation is based on reflectance measurements. The total dot gain $\Delta a_{t o t}$ which includes both physical and optical dot gain, is then given by the difference between the effective dot area, $a_{e f f, R}\left(a_{r e f}\right)$, and the reference one, $a_{\text {ref }}$.

$$
\Delta a_{t o t}=a_{e f f, R}\left(a_{r e f}\right)-a_{r e f}
$$

The Murray-Davies model is also expressed in terms of density to determine the dot gain. The density form of Murray-Davies equation is obtained by using the logarithmic relationship between the density and reflectance.

$$
D=-\log R
$$

By using Equation (3.8) in Equation (3.5) and rearranging the equation, the effective dot area is obtained as in Equation (3.9).

$$
a_{e f f}=\frac{1-10^{\left(D_{p}-D_{N}\right)}}{1-10^{\left(D_{p}-D_{i}\right)}}
$$

where $D_{p}$ is the density of the paper, $D_{i}$ is the density of the ink at full coverage, and $D_{N}$ is the measured density of the sample. 


\subsubsection{Yule-Nielsen Model}

The Yule-Nielsen model is based on the Murray-Davies model [120]. It is actually useful for the color reproduction, which includes optical dot gain effect. In Section 3.4.1, it has been discussed that the effective dot coverage which is calculated by Murray-Davies equation does not correspond to the real physical dot size, as in our measurement the optical dot gain was also included. It is also observed that there is a nonlinear relationship between the measured and the predicted reflectance that could be described with a power function. The exponent $(1 / n)$ is therefore added to the Murray-Davies model to the reflectance values as shown in Equation (3.10).

$$
R(\lambda)^{1 / n}=a_{e f f} R_{i}(\lambda)^{1 / n}+\left(1-a_{e f f}\right) R_{p}(\lambda)^{1 / n}
$$

where the fitting factor $n$ accounts for light scattering in paper and is determined by experiment. The Yule-Nielsen model is often used because it tends to predict printer output somewhat better than the Murray-Davies model [113]. It is obvious that $n=1$ reduces the YuleNielsen to Murray-Davies model. Rukdeschel and Hauser presented that $n=2$ corresponds to a highly scattering substrate [101]. They have also shown that the values of $n$ between 1 and 2 are physically meaningful, while values greater than 2 represent other effects, such as variations in dot density. After experimenting with a variety of papers, halftoning techniques, and area coverages, it has been suggested that $n=1.7$, is an appropriate value when the real $n$ value is unknown [94]. However, $n$ value greater than 2 are often required for modern, high-resolution printers [113].

An example has been suggested in [113] to analyze this statement that Murray-Davies model over-predicts the reflectance. For this purpose in Yule-Nielsen model $n=2$ is selected and $R_{i}(\lambda)$, the ink reflectance at full coverage is replaced by $R_{p}(\lambda) T_{i}^{2}(\lambda)$ in Equation (3.10), where $R_{p}(\lambda)$ is paper reflectance and $T_{i}(\lambda)$ is ink transmittance. Therefore the spectral reflectance of Yule-Nielsen model is transferred to Equation (3.11).

$$
R(\lambda)=\left[a_{e f f, i}\left(R_{p}(\lambda) T_{i}(\lambda)^{2}\right)^{1 / 2}+\left(1-a_{e f f, i}\right) R_{p}(\lambda)^{1 / 2}\right]^{2}
$$

and the following expansion can be performed on Equation (3.11). 


$$
\begin{array}{r}
R(\lambda)=a_{e f f, i}^{2} R_{p}(\lambda) T_{i}(\lambda)^{2}+2 a_{e f f, i}\left(1-a_{e f f, i}\right) R_{p}(\lambda) T_{i}(\lambda)+\ldots \\
\ldots+\left(1-a_{e f f, i}\right)^{2} R_{p}(\lambda)
\end{array}
$$

According to Equation (3.12) the effective dot area in Yule-Nielsen model $(n=2)$ is reduced because $a_{e f f, i}^{2}$ and $\left(1-a_{e f f, i}\right)^{2}$ are both smaller than 1. It has been graphically illustrated in Figure 3.10 for $a_{\text {eff }}=0.5$ that the overall reflectance which is predicted by Yule-Nielsen model $n=2$ is lower than Murry Davies model $n=1$. Generally this expansion is not recommended, because the example is valid only for integer $n$-values. This example is presented only for better understanding of the concept $n$.

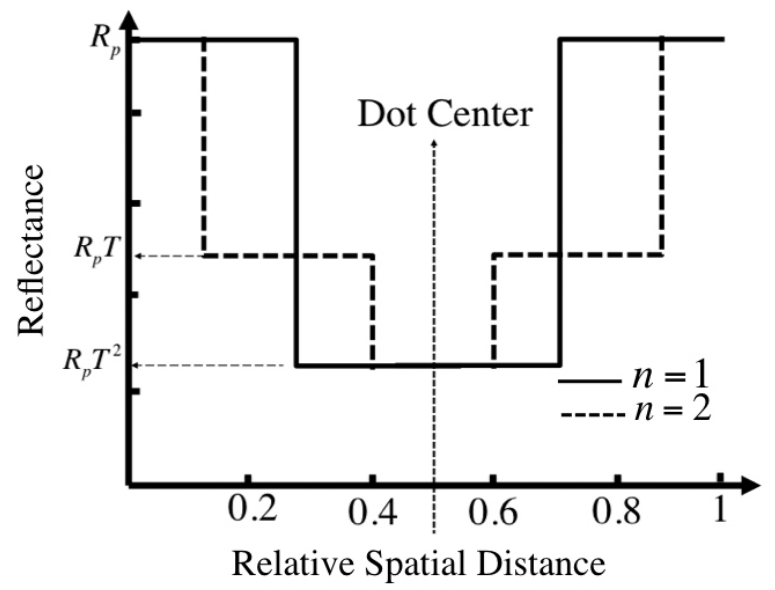

Figure 3.10: Diagram of reflectance computed by Murray-Davies and Yule Nielsen model, $n=2$. The results show that Murray-Davies model over-predicts the spectral reflectance compared to Yule-Nielsen model $n=2$.

In 1996, Arney, et al. [4] proposed a model to approximate the $n$ value as in Equation (3.13).

$$
n \cong 2-e^{-A k_{p}^{\nu}}
$$

where $\mathrm{A}$ is a constant related to the geometry of dot, $k_{p}$ is the inverse frequency at half maximum of the Modulation Transfer Function (MTF), which is the Fourier transform of the PSF (different methods to obtain 
MTF is described in Chapter 6). In Equation (3.13), $\nu$ is the halftone dot frequency with the unit dot/millimeter.

\subsubsection{Expanded Murray-Davies Model}

Arney et al. [2] proposed the expanded Murray-Davies model by considering that $R_{i}$ and $R_{p}$ themselves are functions of area coverage. This fact is based on the observation that the histogram of reflectance values in the halftone image depends on the area coverage. The microscale analysis of halftone prints shows that both the paper reflectance and dot reflectance decrease as the dot area coverage increases $[1,26]$. The final form of the expanded Murray-Davies model is presented by Equation (3.14). However, the reflectance of the ink, $R_{i}(\lambda)$, and paper, $R_{p}(\lambda)$, are functions of area coverages $a_{e f f, i}$, and $a_{e f f, p}$, respectively [113].

$$
R_{i}(\lambda)=R_{g}(\lambda)\left[1-\left(1-T_{i}(\lambda)\right) a_{e f f, i}^{w}\right] \times\left[1-\left(1-T_{i}(\lambda)\right) a_{e f f, i}^{v}\right]
$$

By replacing the effective area $a_{e f f, i}$, with $\left(1-a_{e f f, p}\right)$ in Equation (3.14) the following equation can be obtained,

$$
\begin{array}{r}
R_{p}(\lambda)=R_{g}(\lambda)\left[1-\left(1-T_{i}(\lambda)\right)\left(1-a_{e f f, p}^{w}\right)\right] \times \ldots \\
\ldots \times\left[1-\left(1-T_{i}(\lambda)\right)\left(1-a_{e f f, p}^{v}\right)\right]
\end{array}
$$

where $R_{g}(\lambda)$ is the measured paper reflectance; and $w$ and $v$ are empirically fitting parameters; $v$ is intended to model the softness of the dot edges and $w$ represents the effect of light scattering, as with $n$-value in Yule-Nielsen model. $T_{i}(\lambda)$ represents the ink transmittance, which is equal to $\left(R_{i}(\lambda) / R_{g}(\lambda)\right)^{1 / 2}$ at full area coverage. The effective area of the paper is indicated by $a_{e f f, p}$, which is equal to $\left(1-a_{e f f, i}\right)$. This model works well for a variety of printers, such as offset, thermal transfer, and ink-jet. It has been shown that the expanded Murray-Davies model is as good as the Yule-Nielsen model with adjustable $n$-factor [1].

\subsubsection{Neugebauer Model}

The Neugebauer model is the multiple color ink extension of the MurrayDavies model. The Neugebauer model is proposed to predict the multiple color reflectances of a halftone print [82]. The Neugebauer equation is given by Equation (3.16). 


$$
R(\lambda)=\sum_{i} a_{i} R_{\text {imax }}(\lambda)
$$

where, the summation over $i$, represents the eight Neugebauer primary colors for CMY printing, which are white (unprinted paper), cyan, magenta, yellow, red, green, blue, and combination of the three colors (black). $R_{\text {imax }}(\lambda)$ is the spectral reflectance value of each $i^{\text {th }}$ primary color at full coverage, and $a_{i}$ is the fractional area coverage of each respective colorant. To calculate the fractional area coverage, a common assumption is that the halftone dots are printed randomly on paper. The fractional area coverages of the eight Neugebauer primaries are estimated by Demichel equations shown in Equation (3.17) [21]. The fractional areas for the eight Neugebauer primaries in a three color print are given by,

$$
\begin{aligned}
a_{w} & =\left(1-a_{e f f, c}\right) \cdot\left(1-a_{e f f, m}\right) \cdot\left(1-a_{e f f, y}\right) \\
a_{c} & =a_{e f f, c} \cdot\left(1-a_{e f f, m}\right) \cdot\left(1-a_{e f f, y}\right) \\
a_{m} & =\left(1-a_{e f f, c}\right) \cdot a_{e f f, m} \cdot\left(1-a_{e f f, y}\right) \\
a_{y} & =\left(1-a_{e f f, c}\right) \cdot\left(1-a_{e f f, m}\right) \cdot a_{e f f, y} \\
a_{m y} & =\left(1-a_{e f f, c}\right) \cdot a_{e f f, m} \cdot a_{e f f, y} \\
a_{c y} & =a_{e f f, c} \cdot\left(1-a_{e f f, m}\right) \cdot a_{e f f, y} \\
a_{c m} & =a_{e f f, c} \cdot a_{e f f, m} \cdot\left(1-a_{e f f, y}\right) \\
a_{c m y} & =a_{e f f, c} \cdot a_{e f f, m} \cdot a_{e f f, y}
\end{aligned}
$$

where the indices of $w, c, m, y, m y, c y, c m$, and $c m y$, denote; white, cyan, magenta, yellow, red, green, blue, and black, respectively. It is observable that Neugebauer is a simple three-color extension of the MurrayDavies model [113].

Figure 3.11 shows the three dimensional illustration of Neugebauer model. The inputs of the Neugebauer model are distances along colorant axes and the spectral reflectance of the primary colors. The weights for each primary color are the areas of their respective rectangles, defined by the effective area coverage borders.

It has to be pointed out here that Equation (3.16) is a simple model that is not directly applicable to all practical color print situations. Since in the Murray-Davies equation, it is assumed that $a_{e f f}$ is the physical 


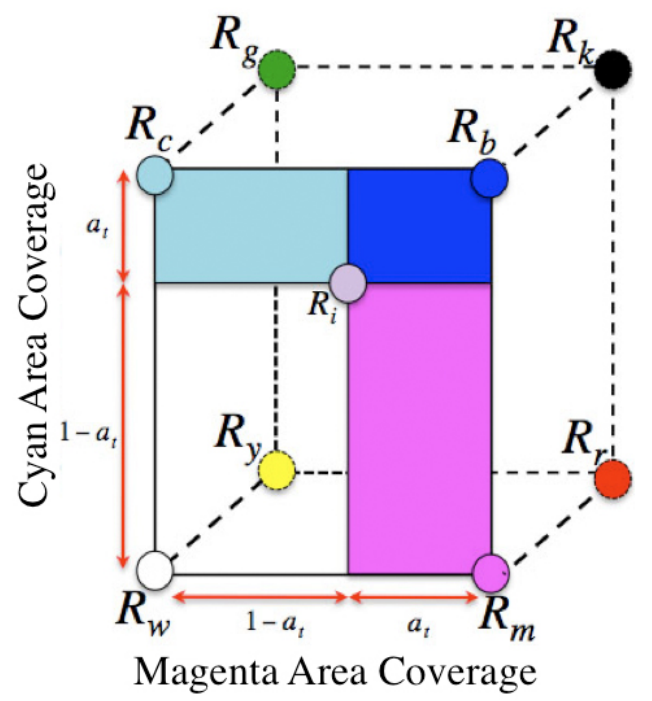

Figure 3.11: 3D illustration of the Neugebauer model. The weights for each primary are the areas of their respective rectangles.

dot coverage, hence in the Neugebauer model the light scattering effect is ignored. In the Neugebauer model it is also assumed that the inks are not penetrating into each other, nor into the paper. In practice, there is always some ink penetration and light scattering effect which cannot be ignored $[5,98]$. One simple way of modifying the Neugebauer model to account for light scattering effect is to follow the Yule-Nielsen modification.

\subsubsection{Yule-Nielsen Modified Neugebauer Model}

The modified Neugebauer model is a combination of Yule-Nielsen and Neugebauer models in Equation (3.18).

$$
R(\lambda)^{1 / n}=\sum_{i} a_{i} R_{\text {imax }}(\lambda)^{1 / n}
$$

where $a_{i}$ is the fractional area coverage of eight Neugebauer primaries discussed in the previous section and shown in Equation (3.17). Here it should be noticed that when fitting the $n$-value, effective area coverages must be recalculated for the Demichel equations each time the $n$-value is changed. 


\subsubsection{Clapper-Yule Model}

To offer an accurate prediction model, one needs to take into account the multiple internal reflections between the air interface and the paper and the lateral light scattering within the paper. In 1953, Clapper and Yule proposed a model for halftone prints to determine surface reflection, internal reflection, and ink transmittance [16,44]. In the Clapper-Yule model the light is reflected many times from the paper surface, both within the ink and paper and also from the background. Figure 3.12 shows the Clapper-Yule model's reflected light from the paper surface, by multiple reflections in a halftone print.

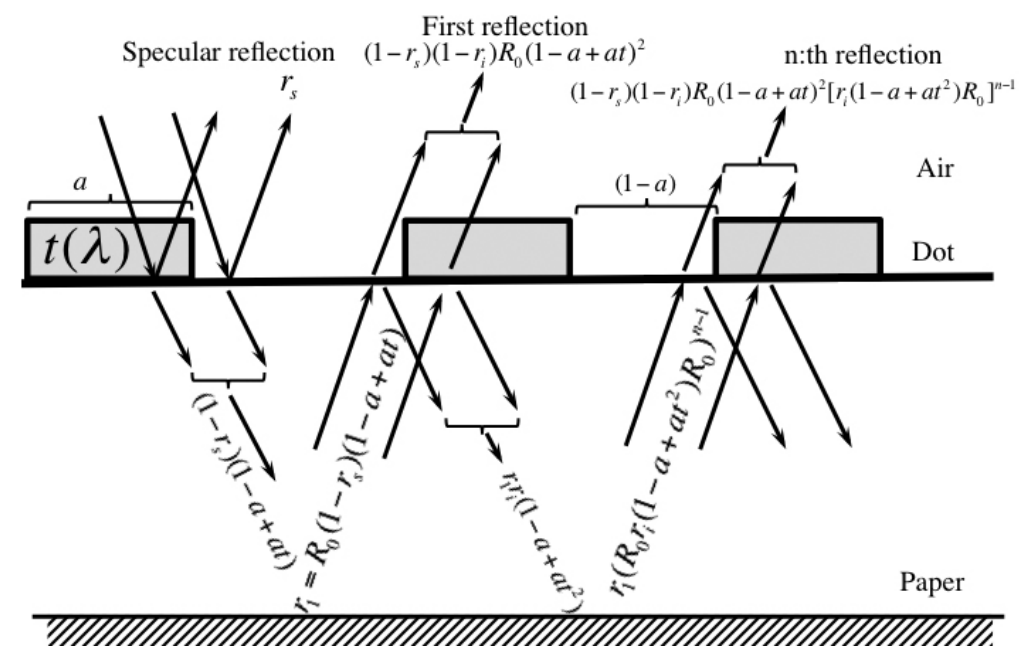

Figure 3.12: Clapper Yule model of the light, halftone dot, and substrate interaction.

The total reflected light is the sum of light fraction that emerges after each internal reflection cycle.

$$
R(a)=K r_{s}+\left(1-r_{s}\right)\left(1-r_{i}\right) \frac{R_{0}(1-a+a t)^{2}}{1-r_{i} R_{0}\left(1-a+a t^{2}\right)}
$$

where $a$ represents the physical dot area coverage, $t$ is the spectral transmittance of the ink, $R_{0}$ is the bulk reflectance of the paper. The factor $K$, ranging from 0 to 1 , gives the fraction of reflected light reaching the detector and depends on measurement geometry. For instruments using 
the $d / 0^{\circ}$ or the $45^{\circ} / 0^{\circ}$ measurement geometry, the specular component can be discarded, i.e. $\mathrm{K}=0$, [46,90]. $r_{s}$ and $r_{i}$ are the Fresnel reflectance values of external and internal reflections in the interface between air and paper [see Equations (3.20) and (3.21)]. Figure 3.13 illustrates the Fresnel reflectance values.

$$
r_{s}=\left(\frac{n_{1} \cos \theta_{i}-n_{2} \cos \theta_{t}}{n_{1} \cos \theta_{i}+n_{2} \cos \theta_{t}}\right)^{2}=\left[\frac{n_{1} \cos \theta_{i}-n_{2} \sqrt{1-\left(\frac{n_{1}}{n_{2}} \sin \theta_{i}\right)^{2}}}{n_{1} \cos \theta_{i}+n_{2} \sqrt{1-\left(\frac{n_{1}}{n_{2}} \sin \theta_{i}\right)^{2}}}\right]^{2}
$$

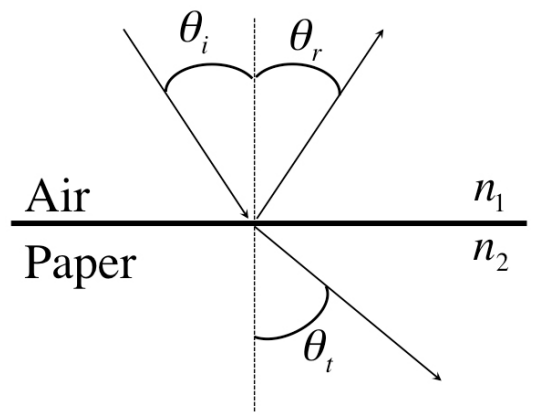

Figure 3.13: Variables used in Fresnel equations.

The Clapper Yule model, like the Yule-Nielsen model, can be combined with the Neugebauer equation to predict the reflectance of multicolorant halftones, see Equation (3.22), [46, 88].

$$
R(a)=K r_{s}+\left(1-r_{s}\right)\left(1-r_{i}\right) \frac{R_{0}\left[\sum_{j=1}^{8} a_{j} t_{j}\right]^{2}}{1-r_{i} R_{0} \sum_{j=1}^{8} a_{j} t_{j}^{2}}
$$

where $a_{j}$ represents the relative amount of colorant $j$ and $t_{j}$ is the corresponding spectral transmittance.

\subsubsection{Kubelka-Munk Model}

The Kubelka-Munk model is a mathematical model based on the model of light propagation used to describe the reflectance of paint coating 
samples [63,64]. In the Kubelka-Munk model several assumptions are considered [22]:

1. The sample is a plane layer of finite thickness with no limitation on width and length.

2. Incident light diffuses homogeneously on the surface of sample.

3. Scattering and absorption are considered as the only interaction between light and medium; polarization and spontaneous emission (fluorescence) are ignored.

4. The sample is assumed isotropic and uniform, and is able to scatter light.

5. Any external or internal surface reflections can occur in the medium.

Since the width and length of the medium is infinite, in the KubelkaMunk model only the thickness direction is incorporated in the equations. The model relates the light intensities $i$ and $j$ in two opposite directions, upwards or downwards.

$$
\left\{\begin{array}{c}
-d i=-(S+K) i d x+S j d x \\
d j=-(S+K) j d x+S i d x
\end{array}\right.
$$

where $x$ indicates distance from the background. $S$ and $K$ are the scattering and absorption coefficients, respectively. At a distance $x$ from the background, a thin layer $d x$ is illuminated by light with intensities $i$ and $j$ from two opposite directions. When the light passes through $d x$, some part of light is absorbed and the rest is scattered. Therefore, the amounts of $i$ and $j$ are decreased by $(S+K) i d x$ and $(S+K) j d x$. On the other hand, the amounts of scattering of the light components $S j d x$ and Sidx are added to intensities $i$ and $j$, as shown in Figure 3.14.

To calculate the light scattering coefficient $S$, it is required to measure the reflectivity $R_{\infty}$ of an infinitely thick (i.e. opaque) medium and the reflectance factor $R$ when the sheet is placed on background $R_{g}$. Then the light scattering coefficient $S$ and the absorption coefficient $K$ are computed as in Equation (3.24) and (3.25).

$$
S=\frac{1}{w\left(\frac{1}{R_{\infty}}-R_{\infty}\right)} \operatorname{Ln}\left(\frac{\left(1-R R_{\infty}\right)\left(R_{\infty}-R_{g}\right)}{\left(1-R_{g} R_{\infty}\right)\left(R_{\infty}-R\right)}\right)
$$




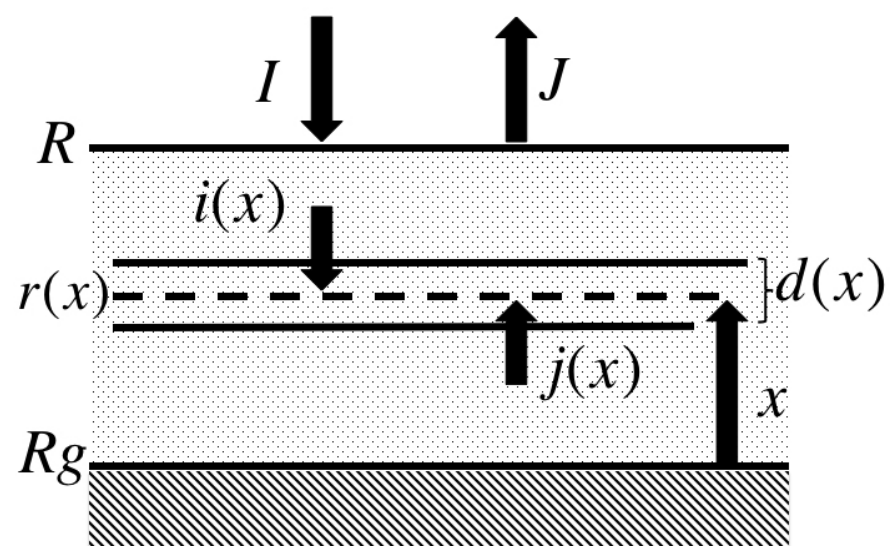

Figure 3.14: The basic model for the derivation of the KubelkaMunk equations.

where $w$ indicates the grammage of the sample which is given in $\mathrm{Kg} / \mathrm{m}^{2}$. The units of $S$ and $K$ are $\mathrm{m}^{2} / \mathrm{Kg}$.

$$
K=\frac{S \cdot\left(1-R_{\infty}\right)^{2}}{2 \cdot R_{\infty}}
$$

The reflectance factor $R$ can be expressed as a function of $S, w, R_{\infty}$, and $R_{g}$ in Equation (3.26).

$$
R=\frac{R_{\infty}\left(\frac{1}{R_{\infty}}-R_{g}\right) \exp \left[S w\left(\frac{1}{R_{\infty}}-R_{\infty}\right)\right]+\frac{\left(R_{g}-R_{\infty}\right)}{R_{\infty}}}{\left(\frac{1}{R_{\infty}}-R_{g}\right) \exp \left[S w\left(\frac{1}{R_{\infty}}-R_{\infty}\right)\right]+\left(R_{g}-R_{\infty}\right)}
$$

The Kubelka-Munk theory has some limitations and in some problematic cases like fluorescence, the model is not accurate enough. The Kubelka-Munk assumptions are too simplified, because in the reality the paper never thoroughly provides all the assumptions. However in some literature, an effort has been made to find a model to revise and improve the Kubelka-Munk theory, due to its explicit form, its simple use and its acceptable prediction accuracy in many cases $[22,118]$. To improve the Kubelka-Munk's results, some numerical methods such as Monte-Carlo, which simulates individual photon path are proposed [22]. 


\subsubsection{Monte-Carlo Simulations}

Photon transport in a medium can be numerically modeled with MonteCarlo simulations or analytically by the radiative transfer (RT) equation. The general radiative transfer (RT) theory is considered for the description of light propagation, scattering and absorption [55]. This theory describes the change of the radiance $d I$ along the propagation path $d s$. The RT equation can be stated as in Equation (3.27) [14],

$$
\frac{d I(s, \theta, \phi)}{d s}=-I(s, \theta, \phi)\left(\sigma_{s}+\sigma_{a}\right)+\frac{\sigma_{s}}{4 \pi} \int_{4 \pi} p\left(u_{i}, u_{s}\right) I(s, \theta, \phi) d w
$$

where $p\left(u_{i}, u_{s}\right)$ is the phase function that describes the probability for scattering in the direction $u_{s}$ at incident direction $u_{i} . \sigma_{s}$ and $\sigma_{a}$ are the scattering and the absorption coefficients, respectively. $\theta$ is the polar angle, $\phi$ is the azimuthal angle, and $w$ is the solid angle. The first term on the righthand side describes intensity reduction due to absorption and scattering. The second term is the contribution to the intensity from scattering [105]. The Kubelka-Munk equation, Equation (3.23) can be also derived from Equation (3.27) if the medium is diffusely illuminated, non absorbing and opaque [83]. Under these conditions the Kubelka-Munk parameters are directly related to the physical scattering and absorption coefficients, with $K=2 \sigma_{a}$ and $S=\sigma_{s}$ [20]. In order to solve Equation (3.27) the Monte-Carlo simulation is used. Monte-Carlo simulation can be used to solve a variety of physical problems. The drawback is that it is far more time consuming. The Monte-Carlo simulations are based on macroscopic optical properties that are assumed to extend uniformly over small units of tissue volume [15]. The light scattering in the substrate leading to optical dot gain has been modeled with the Kubelka-Munk theory $[7,64,93]$ or with Monte-Carlo methods. MonteCarlo simulations allow simulating the spatially resolved reflectance of halftone printed papers. While the Kubelka-Munk theory approximates the light intensity within a medium with two diffuse fluxes, Monte-Carlo simulations solve the general RT equation [14] including single scattering anisotropy [84]. Coppel et al. argued that directional inhomogeneity of uncoated paper limits the applicability of general RT theory using single scattering, independent of the light direction, within the substrate [19]. However, the scattering is more uniform in coatings, and Monte-Carlo simulations can be used successfully to predict the reflectance of halftone printed coated papers, as shown earlier by Sormaz et al. [107]. 


\section{Chapter 4}

\section{Material and Measurement}

Contents

4.1 Introduction $\ldots \ldots \ldots \ldots \ldots \ldots$

4.2 Spectral Measurement . . . . . . . . . 54

4.3 Image Acquisition System . . . . . . . . . . 54

4.3.1 Image Acquisition Setup . . . . . . . . . . . 54

$4.3 .2 \quad$ Color Filters . . . . . . . . . . . . . . . 56

4.3 .3 Gray Scale Image Acquisition . . . . . . . . 58

4.3.4 Color Image Acquisition . . . . . . . . . . 59

4.3.5 Multi-Channel Image Acquisition . . . . . . . 59

4.4 Test Targets and Measurements . . . . . 62

4.4 .1 Test Target $1 \ldots \ldots \ldots$. . . . . . . . . . 62

4.4 .2 Test Target $2 \ldots \ldots \ldots \ldots$. . . . . . . . 64

4.4 .3 Test Target $3 \ldots \ldots$. . . . . . . . . . . . . . . . 64

4.5 Paper Type . . . . . . . . . . . . 65 


\subsection{Introduction}

The reflected light can be measured by both macroscopic or microscopic measurements. In terms of the macroscopic measurement, the spectrophotometer or the densitometer are used to measure the spectral reflectance or the density of the halftone print. On the other hand, the microscopic measurement is based on the measurement of microscale images captured by a high-resolution camera or a microscope. Nowadays, the macroscopic instruments have become relatively fast and more accurate. Therefore most of the printing companies use macroscopic measurements to evaluate the color and tone reproductions [108]. Due to the light scattering effect and ink spreading on the paper surface, the precise modeling of halftone color reproduction is a difficult issue. Most color prediction or tone reproduction models such as Murray-Davies, Yule-Nielsen, Clapper-Yule and Kubelka-Munk are based on macroscopic measurements which were explained in Chapter 3. Throughout this dissertation, for measuring reflectance and transmittance spectra in macroscopic scale, a spectrophotometer has been used.

Microscopic measurement allows us to study the halftone dot shapes, ink spreading, and light scattering effect in micro scale. Due to the fact that microscopic instruments are time consuming and the size of the captured image is large, few models have been proposed to analyze the data obtained by the microscopic measurements $[75,89,92,109]$. In this dissertation, for characterizing the color halftone prints and analyzing the physical and optical dot gain in microscopic scale, a high-resolution camera has been used which will be described in detail in Section 4.3.1.

A thorough calibration of the image acquisition system was carried out by Nyström, to ensure stability and repeatability [85]. With respect to repeatability, temporal stability, and spatial uniformity, all components involved in the image acquisition system were calibrated. The spectral sensitivity of the camera, which can not be derived by direct measurements, is estimated by relating the camera response to the spectral reflectance for a set of carefully selected color samples [86, 87].

This chapter gives all the technical information about the spectral measurements, and image acquisition system for acquiring gray scale, color, and multi-channel images. The test targets and the types of paper used for the future studies presented in the next chapters have been thoroughly presented here. 


\subsection{Spectral Measurement}

Spectrophotometer is a quantitative instrument to measure the transmittance or reflectance factor of a material as a function of wavelength. The spectrophotometer measures the reflection of incident light with some distribution of wavelengths from the sample. The system collects the reflected light by a sensor and refracts it into different spectral components. Then the system digitizes the signal as a function of wavelength, reads it out and displays it through a computer. There are different types of spectrophotometers, based on the detectors geometry and the placement of light sources.

In this study, the spectrophotometer (BARBIERI electronic Spectro $L F P R T)$ with $45^{\circ} / 0^{\circ}$ and $d / 0^{\circ}$ instrument geometry according to ISO 2469 [57] is used to measure the diffuse reflectance and transmittance factors, respectively. In this context, $d$ stands for observation of the light that is diffusely scattered by the sample. The $D_{65}$ illumination with $2^{o}$ observer angle is used to measure the reflectance and transmittance of different samples. The spectrophotometers must be calibrated prior to any sample analysis. The procedure for calibrating spectrophotometer is done by using the white reflectance standard as a perfect reflecting diffuser.

\subsection{Image Acquisition System}

The image acquisition system is an experimental system for controlling and altering the image acquisition setup. While it is possible to capture images of arbitrary objects, the primary usage is to capture microscale images of flat objects, such as substrates [88]. In the following subsections, the image acquisition setup of the high-resolution camera, and the filters used for gray scale, color, and multi-channel image acquisition are described.

\subsubsection{Image Acquisition Setup}

In this section a system is described for the acquisition of multispectral images using a high-resolution camera with a set of optical filters. Figure 4.1 illustrates the high-resolution camera setup, that makes it possible to clearly see the small halftone dots and their surroundings. This high-resolution camera is available at MIT research group labora- 
tory of Linköping University. As it can be seen in Figure 4.1, the paper sample is placed on a plate. This setup is equipped with an electrical motor to move the plate in the $\mathrm{x}-\mathrm{y}$ direction and rotate it around the optical axis. The illumination is provided by a tungsten halogen lamp and transferred by optical fibers. The optical fibers transmit the light through two different paths, from above and below the paper. The reflected images are resulted from the light illuminated at $45^{\circ}$ (adjustable angle) on the paper. The transmitted images are resulted from the light that perpendicularly passes from below the paper. The CCD camera used in the image acquisition system is the Pixelfly HiRes monochrome CCD 'ICX205AL', from PCO.imaging. It has a resolution of $1360 \times$ 1024 pixels, a dynamic range of $12 \mathrm{bits}$, and the available exposure time range from $10 \mu \mathrm{s}$ to $10 \mathrm{~s}$. The CCD camera is combined with macro optics, consisting of enlarging lenses and various extension rings. In our experiments an extension ring is used to provide a resolution of 1.94 $\mu \mathrm{m} /$ pixel, a field of view of $2.65 \mathrm{~mm} \times 1.99 \mathrm{~mm}$, and with a $45^{\circ} / 0^{\circ}$ and $d / 0^{\circ}$ geometries for both microscale image capturing of reflection and transmission.

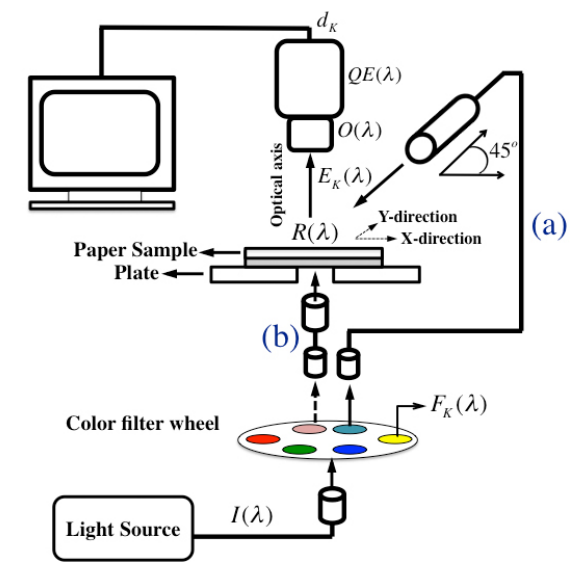

Figure 4.1: The high-resolution camera setup for reflected and transmitted image capturing. The optical fibers transmit the light through two different paths, from (a) above and (b) below paper.

Equation (4.1) describes the linear model of the image acquisition process in case of reflected image capturing.

$$
d_{K}=\int_{\lambda \epsilon V} I(\lambda) F_{K}(\lambda) R(\lambda) O(\lambda) Q E(\lambda) d \lambda+\varepsilon_{k}
$$


where $I(\lambda)$ is the spectral irradiance of the illumination (including the spectral characteristics of lamp as well as the optical fibers used for light transportation). $F_{K}(\lambda)$ is the spectral transmittance of filter $K . R(\lambda)$ is the spectral reflectance of the paper. $O(\lambda)$ is the spectral transmittance of the optics. $Q E(\lambda)$ is the spectral quantum efficiency for the CCD sensor. For channel $K, \varepsilon_{K}$ is the measurement noise and the spectral sensitivity region of the CCD sensor is denoted by $V[40,88]$.

To measure the transmitted light which is illuminated from below, the object transmission, $T(\lambda)$, and the spectral transmission properties of the transmissive plate of the system, $T_{\text {plate }}(\lambda)$ are also taken into account in the model as in Equation (4.2).

$$
d_{K}=\int_{\lambda \epsilon V} I(\lambda) F_{K}(\lambda) T(\lambda) T_{\text {plate }}(\lambda) O(\lambda) Q E(\lambda) d \lambda+\varepsilon_{k}
$$

The validity of the spectral image acquisition model according to Equations (4.1) and (4.2) requires a linear response of the camera, i.e. a camera response proportional to the energy of the incident light. The amplitude of the camera response value, $d_{K}$, will also depend on the aperture size, the exposure time, and on the used focal length. However, this will only affect the total level of incoming radiance to the CCD sensor, that is not wavelength dependent, and does not need to be included in the spectral image acquisition model. The light source and all filter characterizations have been previously described in [88]. The specification of light source and filter transmittance, are measured by using the spectrophotometer PR-650 SpectraScan from Photo research [95]. The spectrophotometer in the system is placed at the same optical axis as the camera. In order to measure the spectral reflectance, a diffuse white reflectance standard is used [23]. The reflectance standard, reflects $99 \%$ $( \pm 1 \%)$ of the illumination, and is placed on the sample's plate. All measurements are performed using the $45^{\circ} / 0^{\circ}$ geometry, at recommended lamp intensity level of $90 \%$, and after an appropriate warm up time for the lamp. The functionality of all components in the image acquisition system is controlled using Matlab interface.

\subsubsection{Color Filters}

In the high-resolution camera setup all filters are placed on a filter wheel. The filter wheel in Figure 4.1 includes 19 different filters with the stan- 
dard diameter of $25 \mathrm{~mm}$ placed in the optical path of the light source. The color standard filters consist of:

- additive color filters (RGB),

- subtractive color filters (CMY),

- a set of 7 broadband interference filters,

- a set of 6 neutral density filters of various optical densities (OD).

Table 4.1 shows the standard filters with their position in the color filter wheel. Although there are 20 positions in the color filter wheel, there is no filter in position $K=1$ in which the light $I(\lambda)$ directly passes through the filter wheel. Equation (4.3) is used to measure the filter

Table 4.1: The standard filters positions in the color filter wheel.

\begin{tabular}{|l|l|c|}
\hline K & Filter & Filter type \\
\hline 1 & --- & No filter \\
\hline 2 & Red & \\
3 & Green & Additive filter set: RGB \\
4 & Blue & \\
\hline 5 & $400 \mathrm{~nm}$ & \\
6 & $450 \mathrm{~nm}$ & \\
7 & $500 \mathrm{~nm}$ & Broadband interference filters \\
8 & $550 \mathrm{~nm}$ & (Denoted by center wavelength) \\
9 & $600 \mathrm{~nm}$ & \\
10 & $650 \mathrm{~nm}$ & \\
11 & $700 \mathrm{~nm}$ & \\
\hline 12 & $0.15 \mathrm{OD}$ & \\
13 & $0.30 \mathrm{OD}$ & \\
14 & $0.40 \mathrm{OD}$ & Neutral Density filters \\
15 & $0.60 \mathrm{OD}$ & (Denoted by optical density) \\
16 & $0.90 \mathrm{OD}$ & \\
17 & 2.50 OD & \\
\hline 18 & Cyan & \\
19 & Magenta & Subtractive filter set: CMY \\
20 & Yellow & \\
\hline
\end{tabular}


transmittance for all the standard filters in the color filter wheel [88].

$$
f_{K}(\lambda)=\frac{I_{K}(\lambda)}{L(\lambda)}
$$

where $I_{K}(\lambda)$ is the spectral irradiance and $L(\lambda)$ is the measured spectral irradiance of the illumination when no filter is used.

\subsubsection{Gray Scale Image Acquisition}

For the gray scale image capturing the color filter wheel is rotated to position $K=1$ corresponding to no filter. The light source is passed through the optical fiber and illuminated on the sample with the $45^{\circ}$ geometry. The sample reflects back the light to the CCD camera. In this case the transmittance of filter $F_{k}(\lambda)$ has no impact on the image acquisition system. Figure 4.2 illustrates the normalized spectral distribution of the illumination system, which is used for gray scale image capturing. The spectral power is very low under $400 \mathrm{~nm}$. The infrared radiation is a common source for errors, as it affects the camera response [40]. To avoid the effect of infrared radiation, the cut-off filter is used that causes a significant drop in the spectral power above $750 \mathrm{~nm}$.

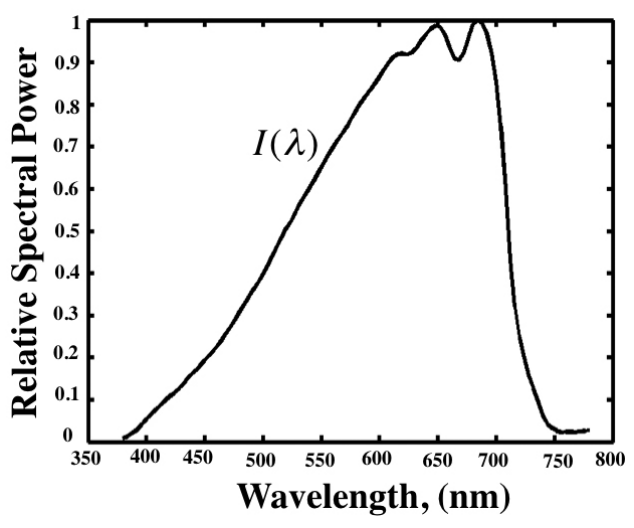

Figure 4.2: Spectral power distribution of illumination system, including the lamp and the fiber optics, measured by using the reference reflectance standard. 


\subsubsection{Color Image Acquisition}

For the color image acquisition with three layers such as RGB or CMY, the color filter wheel rotates to three positions, according to the filter positions in Table 4.1. The light passes through each filter and the reflected light from the sample is captured individually for all filters. By processing the captured images in three different layers the color images are produced.

Color images are captured sequentially and the full color information is acquired in every single pixel and there is no need for any interpolation or demosaicing, as in the case for conventional digital cameras using color filter arrays. Figure 4.3 (a) illustrates the filter transmittance $F_{k}(\lambda)$, for the RGB image capturing. Although the spectral distribution of the illumination in Figure 4.2 has low spectral power in the short wavelength, between $350 \mathrm{~nm}-380 \mathrm{~nm}$, the transmittances of R, G, and B filters are high, especially for the blue filter. The side-lobes for the red and green filters in the short wavelength region, originates from measurement noise due to the low power of the lamp. The side-lobe for the green filter in the long wavelengths shows that the green filter fails in blocking the wavelengths above $700 \mathrm{~nm}$. Figure 4.3 (b) shows the transmittances of the CMY filters. The peaks of filter transmittances are around $90 \%$ for all three filters. Due to noise, the yellow filter also has a side-lobe in the short wavelength region.

\subsubsection{Multi-Channel Image Acquisition}

A set of seven broadband interference filters are used for the acquisition of multi-channel images. The filters are evenly spaced covering the visible spectrum. All the interference filters have a bandwidth of $80 \mathrm{~nm}$ FWHM (full width-half maximum), except for the filter with $400 \mathrm{~nm}$, having $50 \mathrm{~nm}$ FWHM. The intensity of transmitted lights, as it was promised by the manufacturer are between $55 \%$ - $65 \%$ of the intensity of the light source. Figure 4.4 shows the transmittances of these filters with center wavelengths band of $400 \mathrm{~nm}, 450 \mathrm{~nm}, 500 \mathrm{~nm}, 550 \mathrm{~nm}, 600$ $\mathrm{nm}, 650 \mathrm{~nm}$, and $700 \mathrm{~nm}$.

To separate a specific colorant in a color print, it is possible to send light in the reflective band of that color. For this purpose, the multichannel image captured in the seven different wavelengths band can be used. This can be shown with the example of yellow patch given in Figure 4.5. The $25 \%$ reference coverage of yellow dots is captured by 


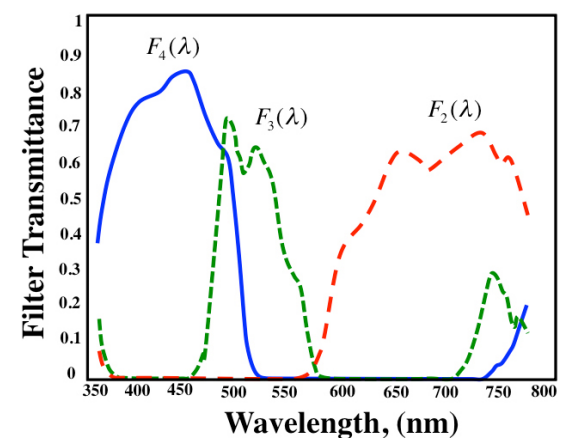

(a)

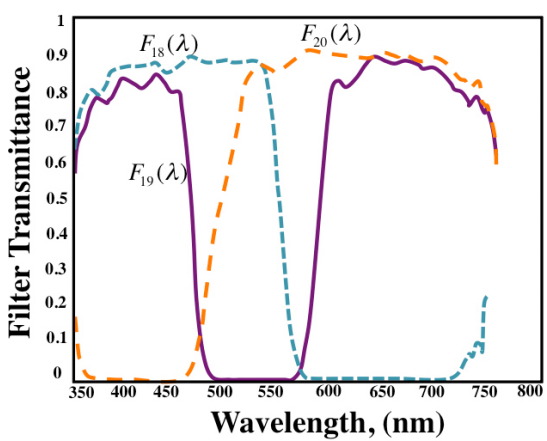

(b)

Figure 4.3: (a) Spectral filter transmittance for the RGB filters.

(b) Spectral filter transmittance for CMY filters.

using high-resolution camera. The first image up to the left in Figure 4.5 is the RGB color image, captured by using filters $K=2,3,4$. The other images are the same yellow patch captured using the seven broadband interference filters. By illuminating the light at $400 \mathrm{~nm}$ which is the absorbing wavelength band of yellow, the dots absorb a large part of the incident light and reflect a small portion of it. In this case the yellow dots become more visible on the paper. Due to the low spectral power of illumination and low spectral transmittance of filter $F_{K=5}(\lambda)$ at $400 \mathrm{~nm}$, the captured image is darker than the images captured with other filters. By approaching the reflective wavelength band of yellow, the yellow dots become pale and in the image captured by $F_{K=11}(\lambda)$ the yellow dots are almost vanished. This method can be used to separate different color inks in two color prints. In Chapter 8 a model to separate two color inks in color print will be described in detail. 


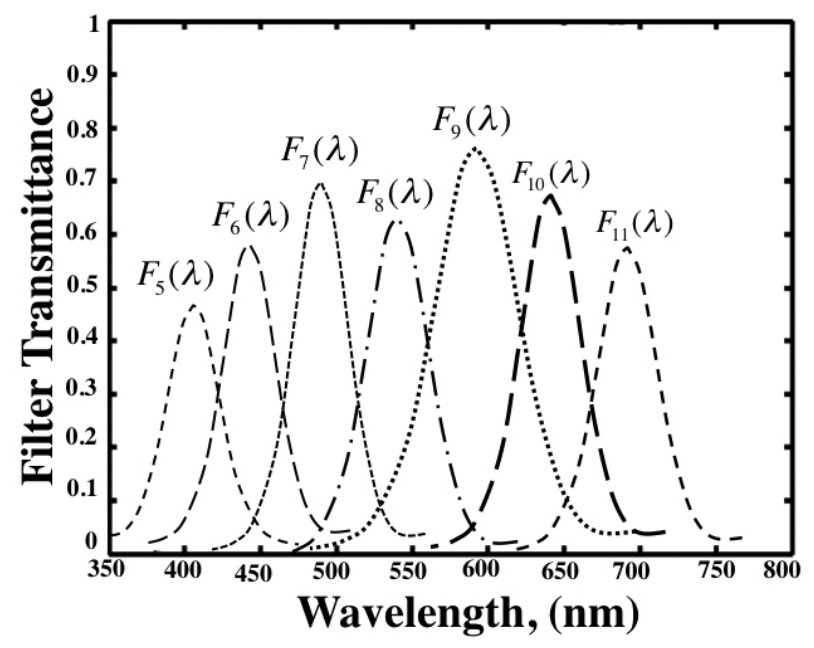

Figure 4.4: Spectral filter transmittance for the seven interference filters, denoted by their center wavelengths, $F_{K}(\lambda), \mathrm{K}=5,6, \ldots, 11$.

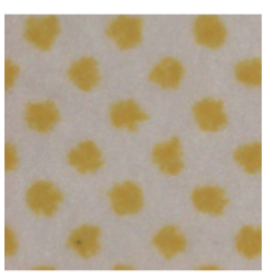

$R G B: F_{2,3.4}(\lambda)$

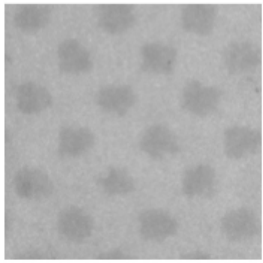

$$
F_{8}(\lambda)
$$

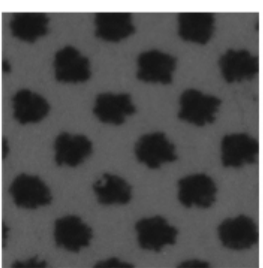

$F_{5}(\lambda)$

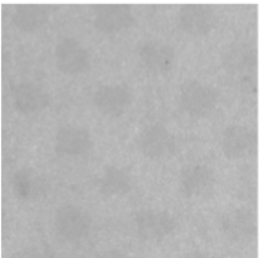

$F_{9}(\lambda)$

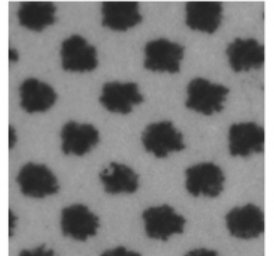

$\overline{F_{6}}(\lambda)$

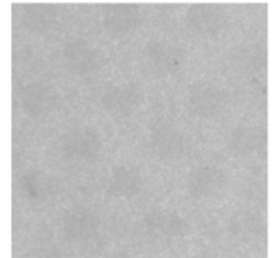

$$
F_{10}(\lambda)
$$

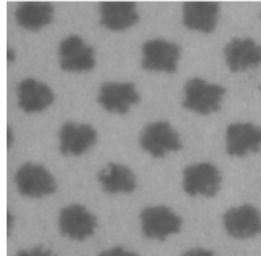

$F_{7}(\lambda)$

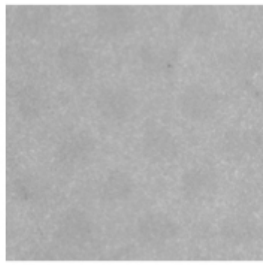

$$
F_{11}(\lambda)
$$

Figure 4.5: $25 \%$ reference coverage of yellow dots captured by using RGB filters $\left(F_{K=2,3,4}(\lambda)\right)$ and seven broadband interference filters $\left(F_{K=5, . ., 11}(\lambda)\right)$. The patch is printed on a coated paper. 


\subsection{Test Targets and Measurements}

In this dissertation, three different test targets are designed and used to fulfill different research goals. All three test targets are printed by offset print press on different type of papers. Each test target has its own specifications and the reason for not using a standard test target, such as Macbeth, ColorChecker chart, or Kodak Q60 targets is that the measurement area and minimum distance for the specterophotometer and high-resolution camera, make it difficult to measure the patches of these small dimensions.

The spectrophotometer with $45^{\circ} / 0^{\circ}$ and $d / 0^{\circ}$ geometry is used to measure the spectral of reflectance and transmittance of all patches. The spectral data are in the range of $380 \mathrm{~nm}$ to $780 \mathrm{~nm}$ sampled at $10 \mathrm{~nm}$ intervals. For all color patches, 14 different microscale images have been captured by using; no filter, the RGB-filters, CMY-filters and the 7 interference filters. It should be noted that the camera response varies depending on the used filter. This is due to the spectral properties of the light source, the transmittance of the filters, and the spectral camera sensitivity. To optimize the dynamic range and the signal to noise ratio, the exposure times are individually calibrated for each color channel [85]. In the following subsections the specifications of each test target is briefly described.

\subsubsection{Test Target 1}

Test target 1 consists of 15 different color charts $(3 \times 5)$. Test target 1 is presented in Figure 4.6. Each chart consists of $81(9 \times 9)$ color patches $\left(\right.$ each $\left.8 \times 8 \mathrm{~mm}^{2}\right)$ with different coverages. The gaps between horizontally and vertically adjacent charts are 0.25 and $1 \mathrm{~cm}$, respectively.

Each set of patches is halftoned with AM, FM2nd, and FM halfoning techniques. For FM2nd three different dot sizes have been used, i.e FM2nd_Big, FM2nd_Medium, and FM2nd_Small. The size of the dots for FM2nd_Big corresponds to those of AM 150 lpi, circular (round) dots. The dot sizes of FM2nd_Medium and FM2nd_Small are then gradually reduced. The print resolution is $1200 \mathrm{dpi}$. In this test target different combinations of dot placement is used: 


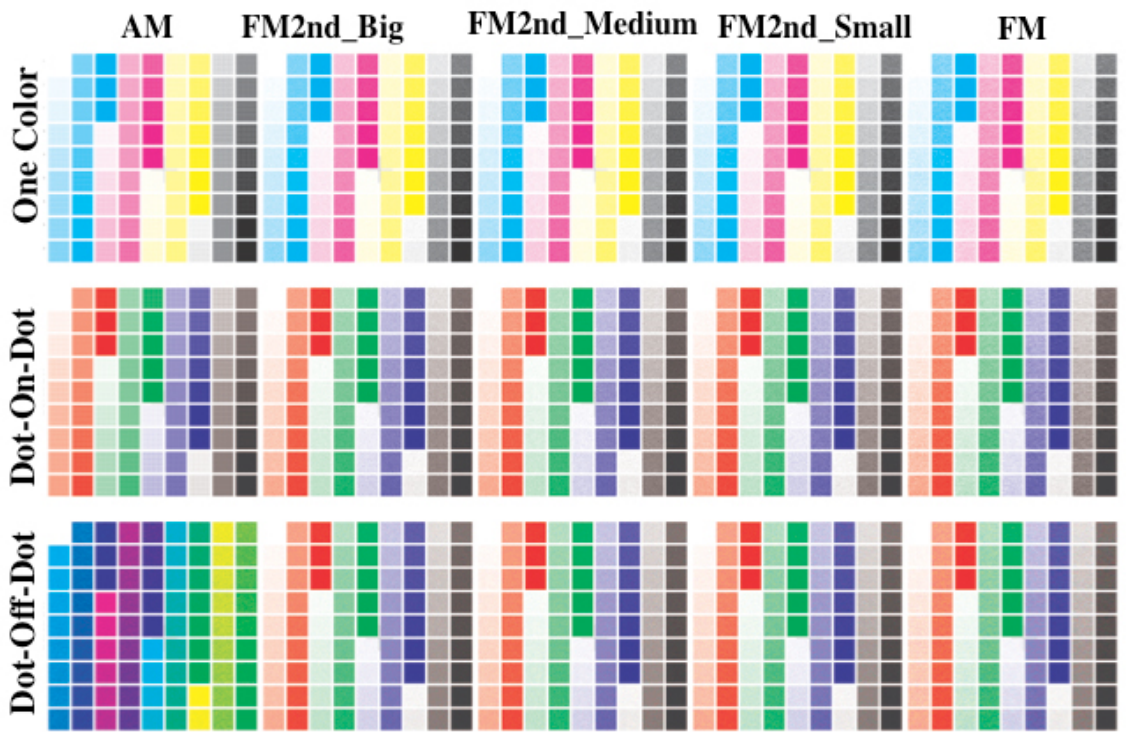

Figure 4.6: The test target halftoned by; AM, FM2nd_Big, FM2nd_Medium and FM2nd_Small and FM. Different combination of dot placement; One color, Dot-on-Dot, and Dot-off-Dot.

1. One Color: All four primary colors; cyan, magenta, yellow, and black, are printed with the following coverages; $0 \%, 2 \%, 5 \%, 10 \%$, $15 \%, 20 \%, 25 \%, 30 \%, 40 \%, 45 \%, 50 \%, 60 \%, 70 \%, 75 \%, 80 \%, 85 \%$, $90 \%, 95 \%, 98 \%$, and $100 \%$, (Row 1 in Test target 1 ).

2. Dot-on-Dot: Cyan, magenta, and yellow are printed on top of each other to make red, green, blue, and black. The same reference coverage as explained above is used for cyan, magenta, and yellow, (Row 2 in Test target 1 ).

3. Dot-off-Dot: Cyan, magenta, and yellow are printed using the dot off dot technique to its limit. When three colors are involved, yellow is printed independent of the other two; because printed yellow on white paper is much less visible than the two other colors [34]. The same coverage as the one color patch is used for cyan, magenta, and yellow, (Row 3 in Test target 1). 


\subsubsection{Test Target 2}

Test target 2 is shown in Figure 4.7. The full tone color stripe of each color is added beside each halftone patch, see the enlargement for black in Figure 4.7. The gray tone values of the full tone coverage are computed by taking the average of the pixel values of these full tone stripes. The gray tone values of the paper are obtained by taking the average of the pixel values of unprinted stripes. All four primary colors; cyan, magenta, yellow, and black, and all secondary colors red, green, blue, and black $(\mathrm{C}+\mathrm{M}+\mathrm{Y})$ are included in this test target. All patches are printed with the following coverages; $0 \%, 5 \%, 10 \%, 15 \%, 20 \%, 25 \%$, $30 \%, 35 \%, 40 \%, 45 \%, 50 \%, 55 \%, 60 \%, 65 \%, 70 \%, 75 \%, 80 \%, 85 \%, 90 \%$, $95 \%$, and $100 \%$. The secondary color patches are also printed using Doton-Dot strategy. Each set of patches is halftoned with three different halftoning techniques as before; AM, FM2nd, and FM. The gap between horizontally and vertically adjacent patches is $0.25 \mathrm{~cm}$.

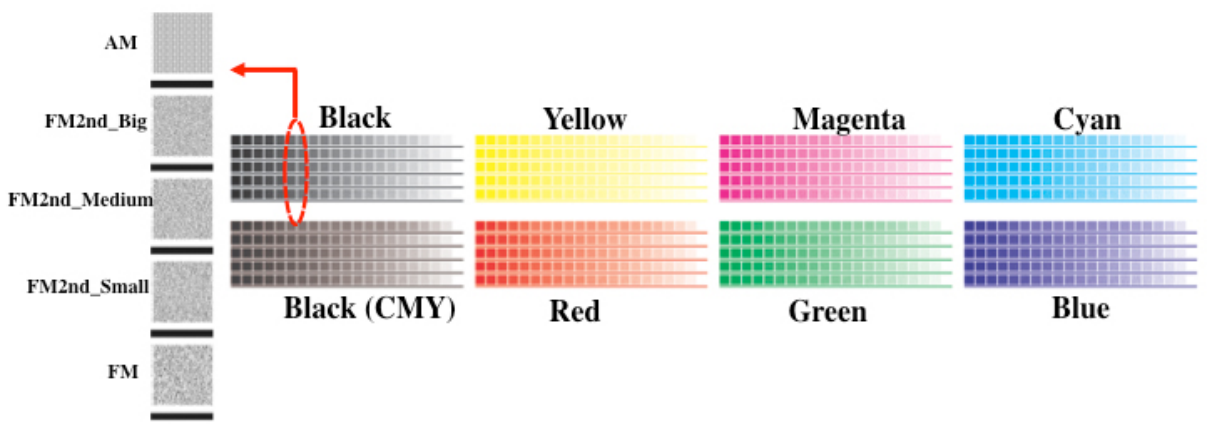

Figure 4.7: The test target halftoned by; AM, FM2nd_Big, FM2nd_Medium and FM2nd_Small and FM. Primary and secondary colors with full tone color stripe of the corresponding color beside each halftone patch.

\subsubsection{Test Target 3}

Test target 3 includes four primary color patches cyan, magenta, yellow, and black, with different reference coverages of $0 \%, 0.5 \%, 1 \%, 3 \%, 5 \%$, $10 \%, 30 \%, 40 \%, 50 \%, 60 \%, 70 \%, 80 \%, 90 \%, 95 \%, 97 \%, 99 \%$, and $100 \%$. All the patches are halftoned by AM halftoning technique at $70 \mathrm{l} / \mathrm{cm}$ (175 lpi). Figure 4.8 illustrates test target 3. 


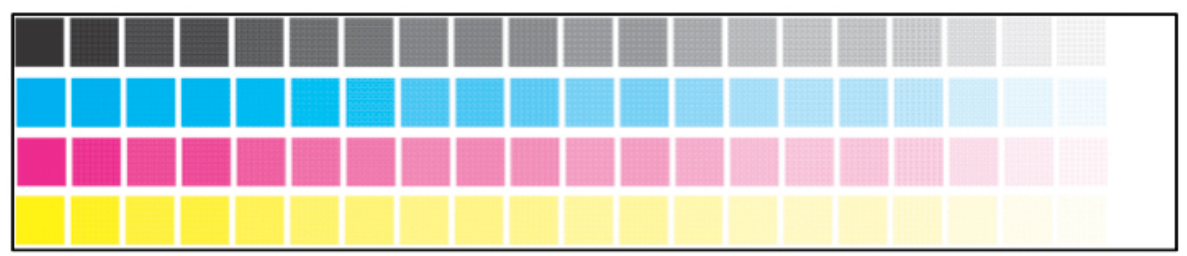

Figure 4.8: The test target includes primary color patches with different coverage, printed on eleven variant samples of coated paper.

\subsection{Paper Type}

Paper can be divided into two major types; coated and uncoated. Coating is a process by which paper or substrate is coated with an agent to improve brightness or printing properties. By applying PCC (Precipitated Calcium Carbonate), chalk (china clay), pigment or adhesive the coating fills the miniscule pits between the fibres in the base paper, giving it a smooth, flat surface which can improve the opacity, lustre and color-absorption ability. Different types of coating are used according to the required paper properties such as; light coated, medium coated, high coated, and art papers. The art paper is used for the high quality reproduction of artwork in brochures and art books [8].

Uncoated paper is typically used for letterheads, copy paper, or printing paper. Most types of uncoated papers are surface sized to improve their strength. Surface sizing of paper refers to the application of starch by means of a size press or film press [8]. Such paper is used in stationary and lower quality leaflets and brochures.

Paper is often specified by its weight. In Europe, and other regions using the ISO 216 [56] paper sizing system, the weight is expressed in grammes per square meter $\left(\mathrm{g} / \mathrm{m}^{2}\right.$ or usually just $\left.\mathrm{g}\right)$ of the paper.

In this dissertation the test targets 1 and 2 shown in Figure 4.6 and 4.7 are printed on two different types of paper; coated and uncoated paper $150 \mathrm{~g} / \mathrm{m}^{2}$. The prints are done by Heidelberg offset print press with the resolution of $2540 \mathrm{dpi}$. The dots are made $2 \times 2$, which reduce the real print resolution to $1270 \mathrm{dpi}$. The reason is to make the halftone dots more stable. An effort is made to keep the ink density constant. Table 4.2 shows the measured densities of primary color inks printed on both coated and uncoated papers given by the printing company. 
Table 4.2: The densities of primary color inks printed on coated and uncoated papers.

\begin{tabular}{|l|c|c|}
\hline Color & Density for coated & Density for uncoated \\
\hline Cyan & 1.62 & 1.10 \\
Magenta & 1.62 & 1.15 \\
Yellow & 1.56 & 1.12 \\
Black & 2.04 & 1.43 \\
\hline
\end{tabular}

All the patches in test target 3 are printed with an offset press on eleven variant samples of coated paper, $65 \mathrm{gr} / \mathrm{m}^{2}$, two $70 \mathrm{gr} / \mathrm{m}^{2}$, three $80 \mathrm{gr} / \mathrm{m}^{2}$, three $115 \mathrm{gr} / \mathrm{m}^{2}$, and two $150 \mathrm{gr} / \mathrm{m}^{2}$ provided by different paper companies. 


\section{Chapter 5}

\section{Microscale Analysis of Halftone Print}

Contents

5.1 Introduction $\ldots \ldots \ldots \ldots \ldots \ldots$

5.2 Image Histogram $\ldots \ldots \ldots . \ldots 9$

5.2.1 Comparison of Reflected and Transmitted Image Histogram . . . . . . . . . . . 71

5.3 Estimation of Total Dot Gain . . . . . . . 73

5.3.1 Using Spectrophotometer . . . . . . . . . . 73

5.3 .2 Microscale Image Approach . . . . . . . . . . . . 74

5.4 Estimation of Physical Dot Gain . . . . . 79

5.4 .1 Arney et al. Approach . . . . . . . . . . 80

5.4.2 Microscale Image Histogram Approach . . . 81

5.5 Summary and Discussion . . . . . . . 85 


\subsection{Introduction}

Characterization of halftone prints gives a good insight to the study of system calibration of print and paper properties. One of the most important phenomena that affects the print result is dot gain, meaning that printed dots appear larger than the dots in the digital bitmap. This is partly due to the ink spreading and ink penetration into the substrate, which results in an enhancement of the physical dot size. This is referred to as the physical dot gain. Lateral propagation of light in paper also causes printed dots to appear larger than their physical size. This phenomenon is referred to as optical dot gain.

Many researches are reported in the literature to separately characterize physical and optical dot gain. Garg et al. [30], Hebert and Hersch [45], Yang and Lundström [119] used transmittance and reflectance value measurement to estimate the physical dot gain effect. Koopipat et al. [61] and Yamashita et al. [116] have reported that the reflected images are images that include both physical and optical dot gain, while the transmitted images only include the physical dot gain effect. In this chapter, by studying the reflected and transmitted image histogram, it will be shown that transmitted images are indeed affected by the optical dot gain [75].

Most available models to predict the outcome of halftone prints are based on macroscopic color measurements, giving the average value over an area that is large relative to the halftone dot size. The aim of this chapter is to go beyond the macroscopic models, and to study halftone print properties on a microscopic level. The research presented in this chapter has appeared in $[74,75,78]$.

\subsection{Image Histogram}

One of the ways to study the characteristics of a halftone print is to analyze the histogram of the microscale image of the halftone print. The image histogram is a plot of the population of different pixel values of the image as a function of the pixel values. In this thesis the pixel values of the microscale images are scaled between zero and one. The value of zero represents black (no reflected light), and the value of one represents white. In this chapter the microscale image of all patches are captured for both reflected and transmitted lights and the histogram are computed for all images. 
Three parameters are commonly needed in the microscopic analysis of halftone images; the effective halftone dot area $a_{e f f}$, the reflectance of unprinted paper $R_{p}$, and the reflectance of the ink dots $R_{i}$. These parameters can be obtained from the histogram of microscale images. However, some factors such as noise in the image, physical spread of the edges of dots, and light scattering in the halftone print influence the estimation of $a_{e f f}, R_{p}$, and $R_{i}$ from the histogram [6]. Figure 5.1 (a) shows the microscale image of $35 \%$ gray (reference coverage in the digital bitmap) including halftone dots (AM round dots, 150 lpi and $1200 \mathrm{dpi}$ ), unprinted paper stripe, and full tone stripe printed on coated paper. Figure 5.1 (b) shows the histogram of all three stripes shown in Figure 5.1 (a).

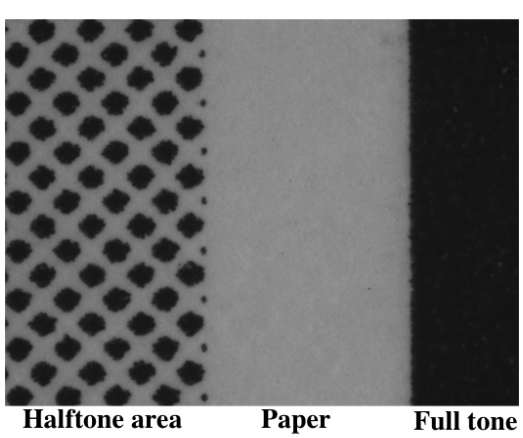

(a)

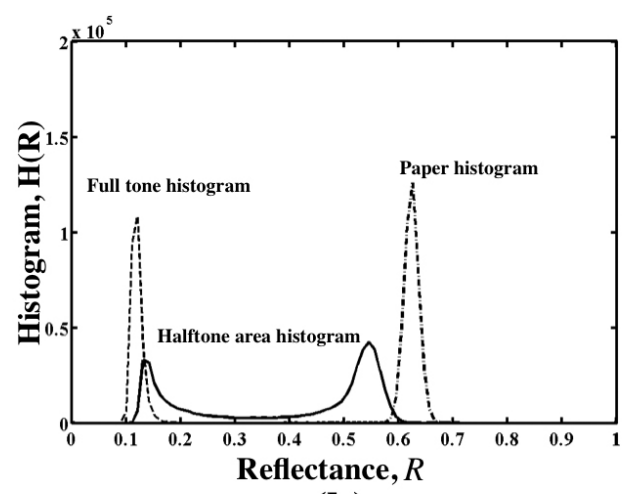

(b)

Figure 5.1: (a) Microscale image with 35\% reference coverage, unprinted paper and 100\% ink stripe. (b) Histogram of the reflected image for $35 \%$ halftone patch including full tone and unprinted paper.

It can be noticed in Figure 5.1 (b) that the two peaks corresponding to the halftoned area histogram do not coincide with the peaks of full tone and paper histograms. Due to the optical dot gain the peak of paper between dots has been shifted to the left, which means that the paper between dots is darker than the paper stripe.

It has been previously claimed in the literature that when the light is perpendicularly illuminated from below the paper, the light will pass through the paper without any scattering $[61,62,109]$. Therefore, they claimed that the reflected images are the images that include both physical and optical dot gain effect, while the transmitted images only have the physical dot gain effect. In contrast, in the following subsection, by 
comparing the reflected and transmitted image histogram, it is shown that light scatters inside the paper even when it is illuminated perpendicularly from below [75]. However, the amount of scattered photons are much less than the case when the light is illuminated from the top of the printed paper by $45^{\circ}$ angle.

\subsubsection{Comparison of Reflected and Transmitted Image Histogram}

When the light is illuminated from below a halftone print, the photons traverse through the ink layer. At the air-ink interface some parts of the photons transit towards the air and a large number of photons reflects back to the paper bulk and scatters again inside the paper [119]. Due to lateral propagation, optical dot gain may occur. Figure 5.2 illustrates how the photons are scattered when they are perpendicularly illuminated from below the paper.

Diffuse Transmittance

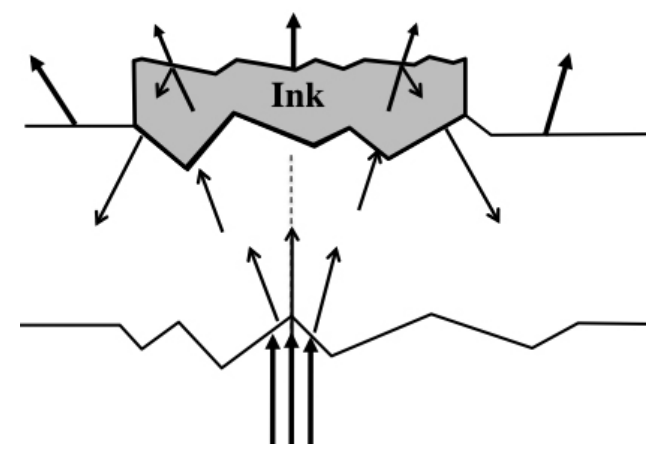

Figure 5.2: Diffuse transmittance when the light is illuminated perpendicularly.

Figure 5.3 (a) and (c) illustrate the reflected and transmitted images for the $35 \%$ (reference coverage) halftone patch and an unprinted stripe, respectively. In order to magnify the dots in these two figures, a small part of the original image have been shown. The histograms of the halftoned area and the paper area are separately plotted. Figure 5.3 (b) and (d) show that there are three peaks corresponding to the unprinted stripe $\left(R_{\mathrm{p}}\right.$ and $\left.T_{\mathrm{p}}\right)$, paper between dots, and ink $\operatorname{dots}\left(R_{i}\right.$ and $\left.T_{i}\right)$ for both reflected and transmitted images. The relative height of the unprinted stripe peak depends on the size of the unprinted area. 
It is obvious in Figure 5.3 (b), that due to the optical dot gain the peak of the paper between dots has been shifted to the left in comparison to the peak of the bulk paper. The histograms of the transmitted image in Figure 5.3 (d) show that the peak of the paper between the dots has also been slightly shifted to the left compared to the peak of the bulk paper. This shows that although the transmitted image includes less optical dot gain compared to the reflected image, one cannot say that there is no optical dot gain included in the transmitted image [75].

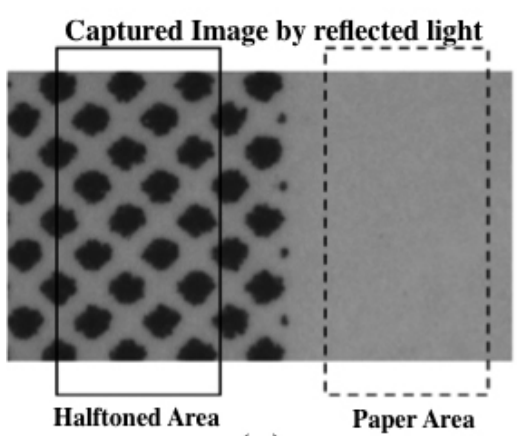

(a)

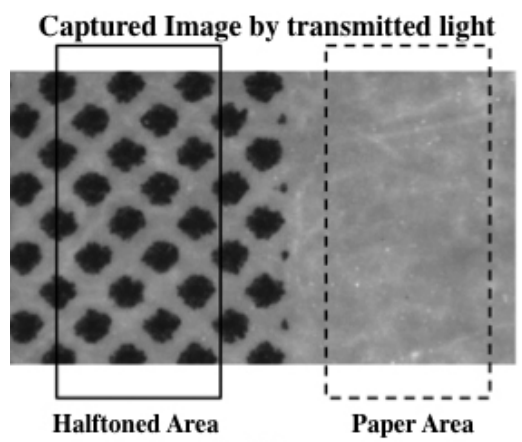

(c)

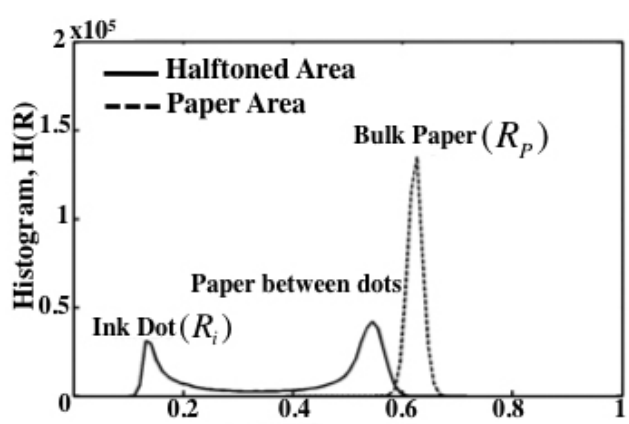

Reflectance, $R$

(b)

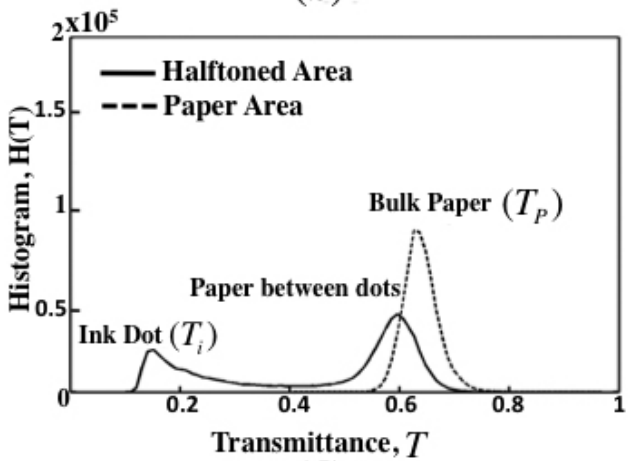

(d)

Figure 5.3: (a) Reflected image for $35 \%$ halftone patch. (b) The histogram of halftoned and paper area of reflected image. (c) Transmitted image for $35 \%$ halftone patch. (d) The histogram of halftoned area and paper of transmitted image. 


\subsection{Estimation of Total Dot Gain}

Characterization of total dot gain is an important issue in the study of paper properties and print characteristics. Most of the proposed models in literature use spectral measurements to calculate the lateral propagation of light into the paper substrate (optical dot gain) and the spreading of the inks (physical dot gain) according to their superposition with other inks $[3,25,47,107,117]$.

In this section, three different approaches for computing the total dot gain are described. One of the approaches is based on macroscopic measurement, using reflectance spectrum measured by the spectrophotometer and the other two are based on microscopic measurement using reflected microscale images captured by the high-resolution camera.

\subsubsection{Using Spectrophotometer}

In order to measure the reflectance spectrum the spectrophotometer mentioned in Section 4.2 is used and calibrated for each patch individually. For this purpose the reflectance spectrum has been measured for 21 gray patches from test target 2 (Section 4.4.2), with different reference coverages of $0,5,10, \ldots, 95,100 \%$. By minimizing the $\Delta$ RMS between the calculated and measured reflectance of Murray-Davies Equation (3.6), $a_{e f f, R}\left(a_{r e f}\right)$ can be found for each reference coverage. It means that $a_{e f f, R}\left(a_{r e f}\right)$ is fitted for each halftone patch to make the measured and calculated spectra as identical as possible. Figure 5.4 (a) shows the reflectance spectrum computed by the Murray-Davies equation, and the reflectance spectrum measured by the spectrophotometer for a $35 \% \mathrm{AM}$ $150 \mathrm{lpi}$ halftone patch. It is obvious that the model works very well for reflectance spectra estimations for black ink. By using Murray-Davies model, the total dot gain has been calculated, Equation (3.6). Figure 5.4 (b) shows the total dot gain that is obtained from the reflectance spectra of 21 halftone patches.

Table 5.1 shows both maximum and average of $\Delta$ RMS and $\Delta E_{a b}$ between the computed and measured spectra for all patches. Small $\Delta E_{a b}$ clearly verify that the Murray-Davies equation can be used to calculate the total dot gain from reflectance spectra for black ink. Also note that the smallest $\Delta \mathrm{RMS}$ does not necessarily result in the lowest $\Delta E_{a b}$, but small $\Delta E_{a b}$ in Table 5.1 indicates that the calculated spectra are very close to the measured ones viewed by human eye. 


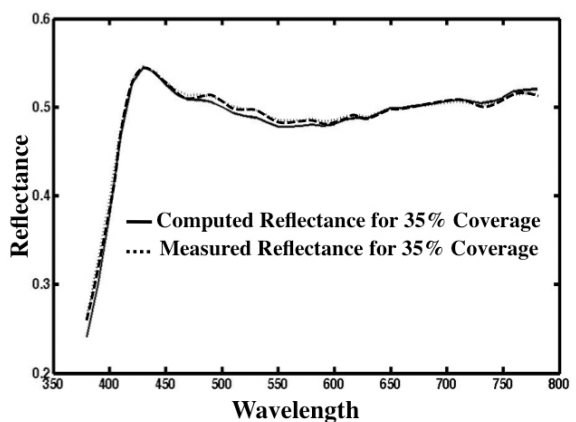

(a)

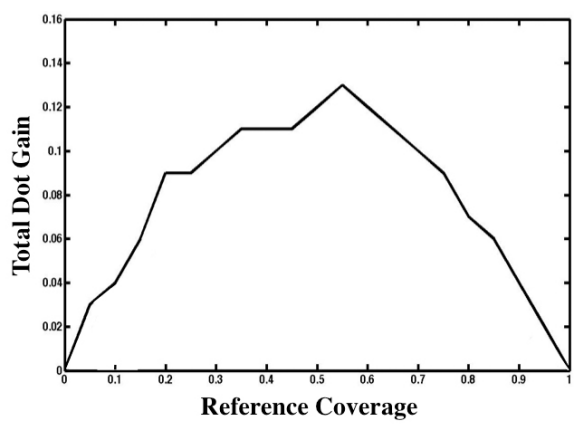

(b)

Figure 5.4: (a) The computed and measured reflectance spectra for $35 \%$ halftone patch. (b) The total dot gain computed by the spectrum approach.

Table 5.1: The differences between computed reflectance spectrum and measured spectrum for all 21 coverages.

\begin{tabular}{|r|c|}
\hline Difference & Reflectance \\
\hline $\max (\Delta \mathrm{RMS})$ & 0.0064 \\
\hline$a v e(\Delta \mathrm{RMS})$ & 0.0040 \\
\hline $\max \left(\Delta E_{a b}\right)$ & 1.1487 \\
\hline ave $\left(\Delta E_{a b}\right)$ & 0.5564 \\
\hline
\end{tabular}

\subsubsection{Microscale Image Approach}

The idea of using microscale images to characterize the dot gain is already examined by Arney, et al. [2]. In their approach an unprinted (paper) stripe is added to the side of the halftoned area in order to find the average value of the paper reflectance. In this section, two approaches are proposed to obtain the total dot gain from the microscale images. The first approach is to use an unprinted stripe and a black stripe, i.e. $100 \%$ ink, placed at the side of each halftone patch. In the second approach, another model that does not require the black stripe is presented. In the latter approach the gray tone of $100 \%$ ink for each patch is calculated by the histogram of the corresponding halftone patch and the unprinted paper stripe. 


\section{- With Black Stripe}

In this approach an unprinted stripe and a black stripe are placed at the side of all halftone patches. Figure 5.1 (a) shows the microscale image consisting of a halftoned patch, unprinted, and $100 \%$ ink stripes. The gray tone of paper and $100 \%$ ink is changed from one captured patch to the next due to different acquisition conditions. The two narrow stripes of paper and $100 \%$ ink are placed beside each patch to make sure that the correct gray tone values for paper and $100 \%$ ink are used for each patch. The gray tones of the paper and the full tone coverage are computed, by taking the average of the pixel values of the unprinted and black stripes, respectively. The total dot gain can be estimated by putting these averages in Murray-Davies equation,

$$
a_{e f f, R}\left(a_{r e f}\right)=\frac{R_{a v e}\left(a_{r e f}\right)-R_{p}}{R_{i}-R_{p}}
$$

where $R_{\text {ave }}\left(a_{\text {ref }}\right)$ is the average of the halftone patch with the reference coverage $a_{r e f}$. The averages of the unprinted and $100 \%$ ink, are denoted by $R_{p}$ and $R_{i}$ respectively. The effective dot area including the optical dot gain is given by $a_{e f f, R}\left(a_{r e f}\right)$. Figure 5.5 shows the total dot gains estimated by using the spectrum and the microscale image (with black stripe) approaches. As seen in Figure 5.5 , the estimations are very close, with a maximum difference of around $2 \%$. This method is a way to calculate the total dot gain. One issue with this method is that a black stripe beside each patch is required. In the following section, a new model to obtain the total dot gain without having a black stripe beside the halftone patches is presented.

\section{- Without Black Stripe}

In this part, it is assumed that there is no black stripe beside each patch. In order to find the gray tone value of $100 \%$ ink, the histogram obtained from the reflected image of the halftoned patch is used. Figure 5.3 (b) shows the histogram of the reflected image for the $35 \%$ halftone patch and its adjacent unprinted paper stripe, respectively. The reflected image histogram consists of three peaks corresponding to the unprinted stripe $R_{p}$, paper between dots, and ink dots $R_{i}$.

When light enters the paper between dots, it may be scattered 


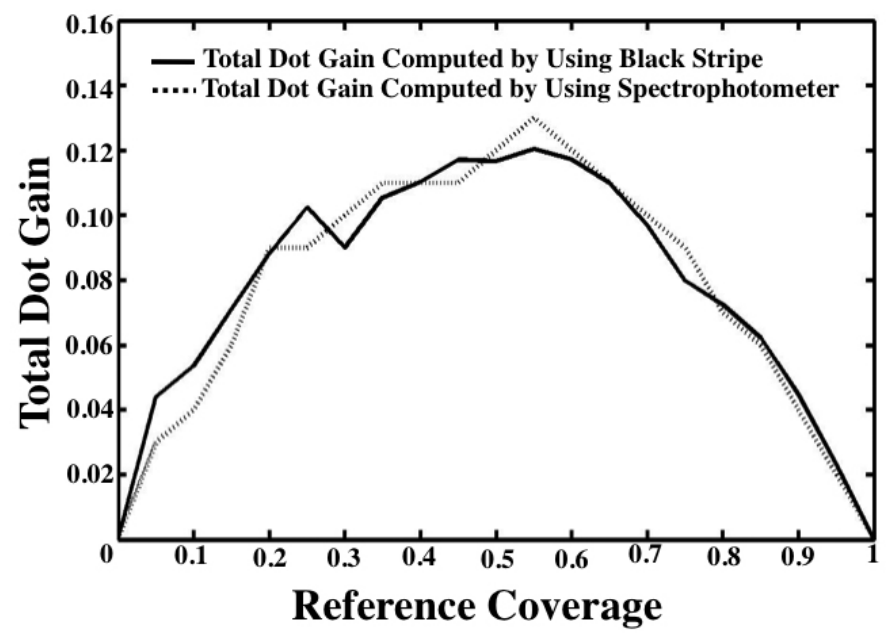

Figure 5.5: Total dot gain estimated by microscale image and spectrum approach.

laterally and emerge under dots or the paper between dots. In this case, the reflectance value of $R_{i}$ is higher than the full tone ink at $a_{e f f}=1$, and the reflectance value of $R_{p}$ is lower than the paper at $a_{e f f}=0$. The significance of these scattered photons is that the overall reflectance of the dots and paper between dots is a function of the relative size of the dots. Thus, $R_{i}$ and $R_{p}$ are not really constant but they are functions of $a_{\text {eff }}[2,91]$. Figure 5.6 illustrates the histograms of halftoned patches with different reference coverages beside unprinted stripe. When the dots get larger, then the space between dots gets smaller. Due to the light scattering effect, the paper between dots gets darker, hence the peak for the paper between dots is shifted more to the left in comparison to the peak from the smaller dots. In this approach, in order to find $R_{p}$ the average value of unprinted stripe is used as before. Now the question is how to use the histogram to find the average gray tone of $100 \%$ ink, $R_{i}$. For this purpose a threshold, $R_{t}$, by use of the reflected histogram of the halftoned area is found to separate the full tone dots from the paper. The gray tone of full tone ink is located around the left peak in the histogram. By traveling towards the paper (as the gray tones become lighter) a rapid drop takes place, which indicates that the dot area finishes. Therefore, the chosen threshold is where the histogram drops the 


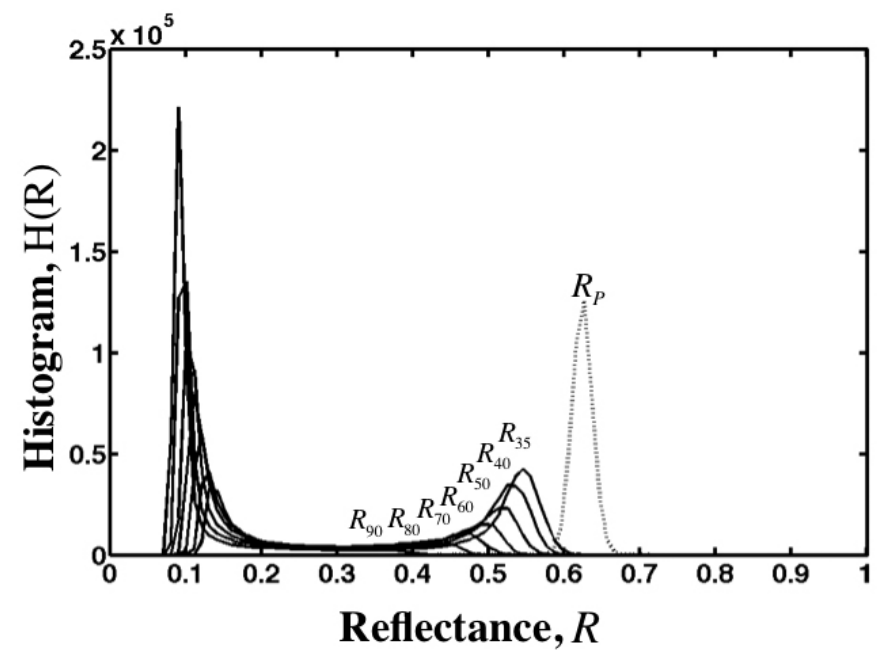

Figure 5.6: Histograms of halftoned patches with different reference coverage beside unprinted stripe.

most. Mathematically, it means that the threshold is where the first minimum of the first derivative of the histogram occurs, which is also equivalent with saying that the threshold is where the second derivative is zero and the curve of the histogram switches from convex down to convex up. This means the threshold is where the second derivative intersects with the reflectance axis of the histogram for the first time while going from negative to positive. Figure 5.7 shows the reflectance histogram of the $35 \%$ halftoned patch including only unprinted stripe, and its respective second derivative. As mentioned, in this approach the zero of the second derivatives of the histograms, see Equation (5.2), is used to find the threshold $R_{t}$.

$$
H^{\prime \prime}(R)=\frac{d^{2} H(R)}{d R^{2}}=\frac{d}{d R}\left(\frac{d H(R)}{d R}\right)
$$

In Equation (5.2), $H(R)$ and $H^{\prime \prime}(R)$, denote the reflectance histogram and its second derivative. When the second derivative becomes zero, the histogram curve will change its convexity direction. The solid curve in Figure 5.7 illustrates the second derivative of the histogram. In Figure 5.7, the first zero value is found where the second derivative changes sign from negative to positive, [see 


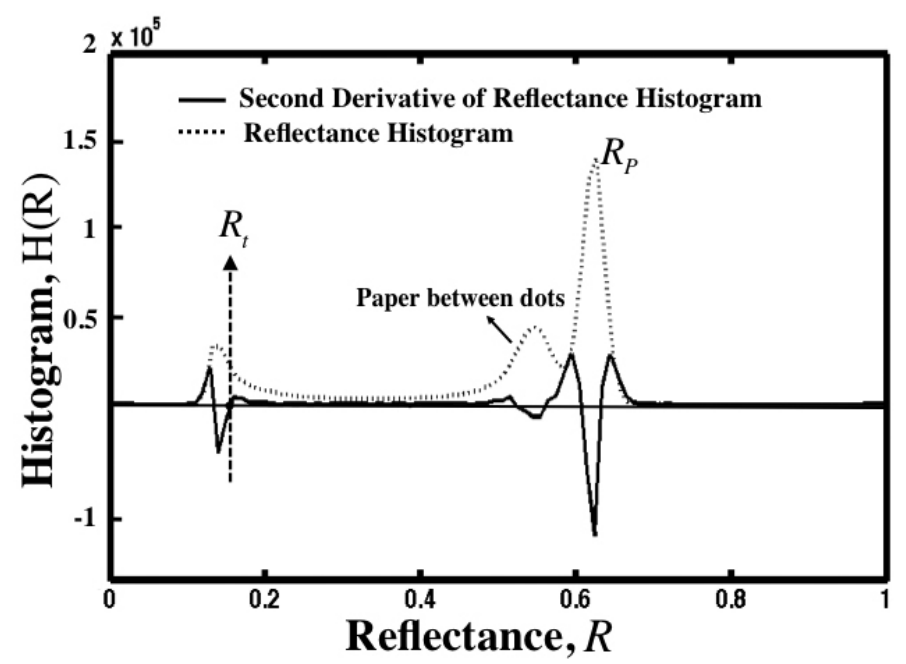

Figure 5.7: The reflectance histogram (dashed line) and its second derivative (solid line).

$\left.R_{t}\right]$. The gray tone values smaller (darker tones) than this threshold define the dot area. The gray tone value of full tone ink for reflected images can now be calculated by Equation (5.3). As the measurement setup varies from patch to patch, $R_{i}$ should be calculated for each patch individually.

$$
R_{i}=\frac{\int_{0}^{R_{t}} R \cdot H(R) d r}{\int_{0}^{R_{t}} H(R) d r}=\frac{\sum_{R=0}^{R_{t}} R \cdot H(R)}{\sum_{R=0}^{R_{t}} H(R)}
$$

The total dot gain can then be approximated by Murray-Davies equation, see Equation (5.1). Figure 5.8 shows the total dot gains obtained by the two approaches presented in this section, namely the microscale image approach with and without the black stripe. It is observable from the figure that the estimations are very close.

So far we have only illustrated the average numerical value of the total dot gain. By the use of microscale images, it is possible to graphically illustrate how the dot gain behaves. This illustration is also useful to characterize the properties of different papers. Figure 5.9 (a) shows the reflected image of a $35 \%$ halftone patch. Since the average value of 


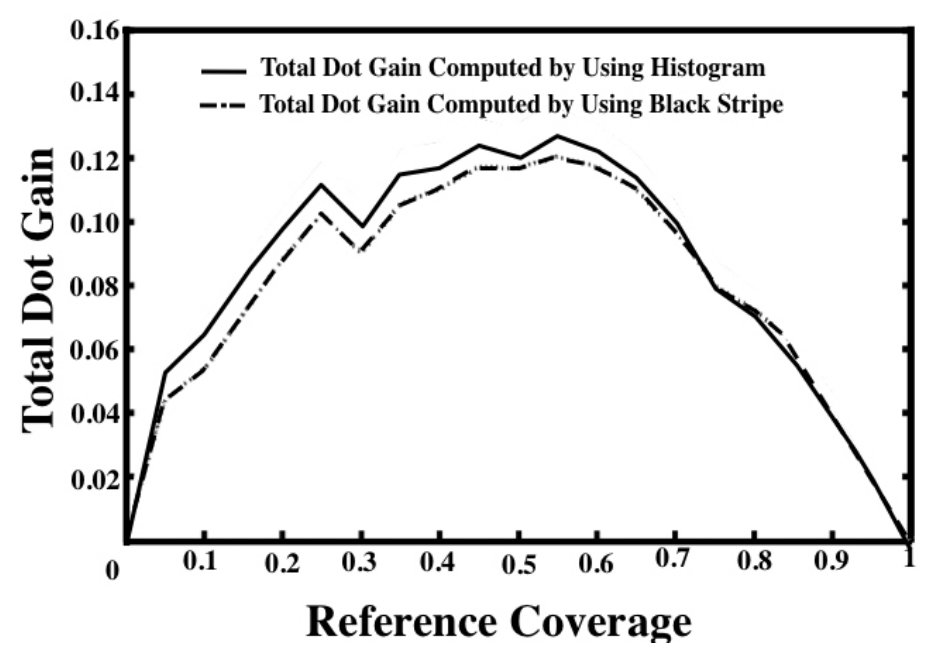

Figure 5.8: Total dot gain estimated by the microscale image approaches, solid line: without black stripe, dashed line: with black stripe.

the total dot gain at $35 \%$ is already estimated, it would be possible to find a threshold to separate the dots from the paper. Assume that the reflected image of the halftone patch at $35 \%$ is denoted by $I M_{35}$, and the effective dot coverage calculated by Murray-Davies is denoted by $a_{e f f, 35}$. The threshold $T$ can be found by Equation (5.4).

$$
\operatorname{mean}\left(I M_{35}<T\right)=a_{e f f, 35}
$$

The result of the threshold operator $\left(I M_{35}<T\right)$ is an image with the same size as $I M_{35}$, which is one in the pixel positions where the pixel value in $I M_{35}$ is less than $\mathrm{T}$ and zero elsewhere. The average of the pixel values is calculated by the (mean) operator. Figure 5.9 (b), shows the effective dot area with an average denoted by $a_{e f f, 35}$, which includes the physical and optical dot gains.

\subsection{Estimation of Physical Dot Gain}

As discussed, the color of a halftone print is affected by ink spreading and lateral light scattering in the substrate, making printed dots appear larger. Characterization of physical and optical dot gain is crucial for the graphic arts and paper industries. Arney et al. [2] proposed an approach 


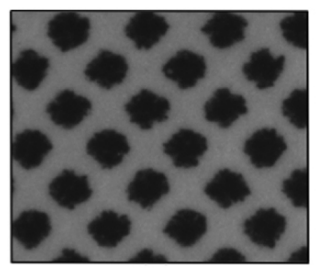

(a)

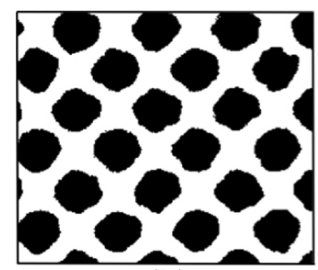

(b)

Figure 5.9: (a) The microscale image of $35 \%$ halftoned patch printed on coated paper. (b) The effective dot area shape.

to estimate the transition reflectance between dot and paper which experimentally can be found by using vertical and horizontal line scans across the halftone dots in the microscale images and averaging several transition reflectances. In the following subsections, first the Arney et al. model and second a new approach are presented to separate the physical and optical dot gain by analyzing the reflected microscale images. This approach is referred to as the "Microscale Image Histogram" (MIH) approach.

\subsubsection{Arney et al. Approach}

As explained in previous section, the average reflectance value of halftoned patch $R_{\text {ave }}$, paper $R_{p}$, and $100 \%$ ink $R_{i}$ can be derived from the histogram of the microscale image. The average of reflectance $R_{\text {ave }}$, corresponding to the microscopic measurement, is given by the integral in Equation (5.5). Notice that here it is assumed that the reflectance histogram is normalized.

$$
R_{\text {ave }}=\int_{0}^{1} R \cdot H(R) d R
$$

Equation (5.5) can be rewritten as Equation (5.6), consisting of two parts corresponding to the contribution of ink and paper, respectively.

$$
R_{\text {ave }}=\int_{0}^{R_{t a}} R \cdot H(R) d R+\int_{R_{t a}}^{1} R \cdot H(R) d R
$$

In Equation (5.6), $R_{t a}$ is a threshold value, defining the transition between dots and paper. The reflectance of the ink $R_{i}$ and the paper $R_{p}$ are approximated by the peak values from the histogram, which transfer Equation (5.6) into Equation (5.7). 


$$
R_{a v e}=R_{i} \int_{0}^{R_{t a}} H(R) d R+R_{p} \int_{R_{t a}}^{1} H(R) d R
$$

The two integrals in Equation (5.7) are the area fractions for the ink, $a_{e f f}$, and the paper, $a_{p}=1-a_{e f f}$. It is evident that Equation (5.7) expresses the Murray-Davies equation. The physical dot area can be computed from the histogram, using a threshold value $R_{t a}$, as the border between the ink and the paper, as shown in Equation (5.8).

$$
a_{p h y}=\frac{\int_{0}^{R_{t a}} H(R) d r}{\int_{0}^{1} H(R) d r}
$$

In Arney et al. model [2], the transition point between dot and paper is defined as the region of maximum rate of change in pixel value $d R / d x$, which is found by moving from the center of a dot to the center of the paper in the microscale image. The threshold $R_{t a}$ is found, by vertical and horizontal line scanning across the halftone dots. Figure 5.10 shows a line scan across halftone dots for a $35 \%$ AM halftone patch. According to their approach, an edge is where the tone variation is large, thus, the transition points are the positions with the steepest slope in reflection between dot and paper which have been marked by crosses in Figure 5.10. The threshold $R_{t a}$, is then the average reflectance values of these points.

\subsubsection{Microscale Image Histogram Approach}

In this part a simple approach based on the reflected microscale image histogram is proposed to estimate the physical dot area of the halftone print. As can be seen in Figure 5.10, by moving few pixels away from the steepest slope points the reflectance values vary drastically (see the two vertical dashed lines in Figure 5.10). Therefore, it can be concluded that the number of pixels having the same reflectance value as the steepest slope points, is minimum. Since a histogram shows the population of different pixel values, then the minimum point of the histogram corresponds to the steepest slope in Arney et al. model. Figure 5.11 (a) shows a microscale image of a halftone patch at $35 \%$ reference coverage and an unprinted stripe. Figure 5.11 (b) shows the histogram of Figure 


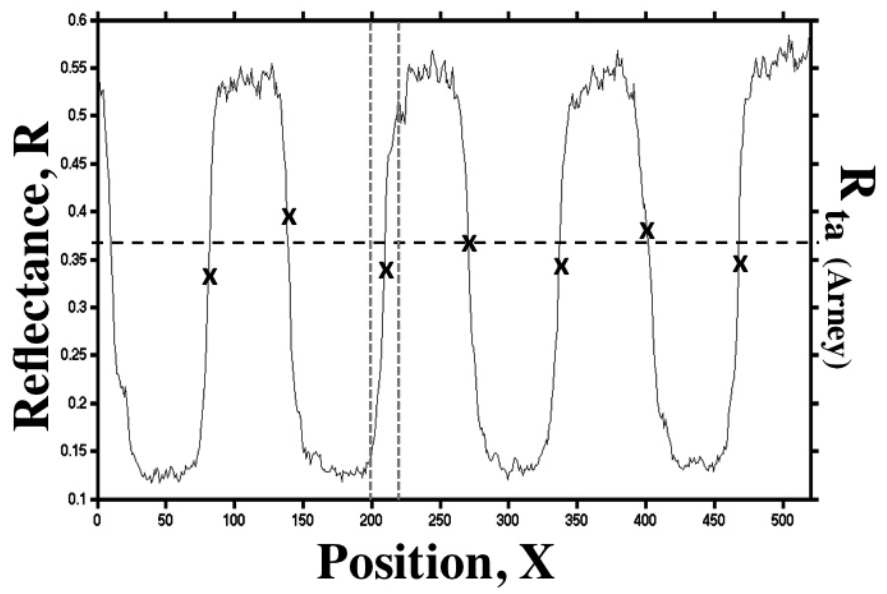

Figure 5.10: Illustration of a line scan (solid line) across halftone dots, with the maximum rate of change and the resulting threshold value (dashed line).

5.11 (a), with three peaks corresponding to the unprinted stripe $R_{\mathrm{p}}$, paper between dots, and ink dots $R_{i}$.

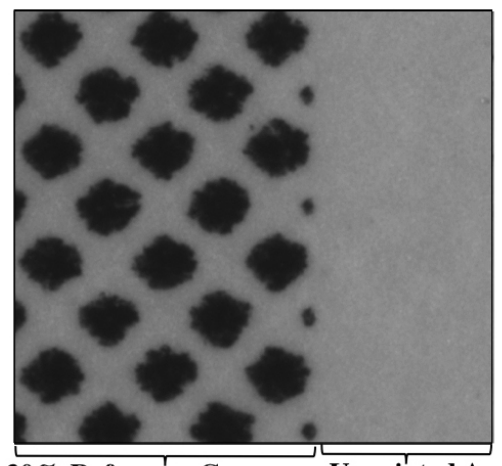

30\% Reference Coverage Unprinted Area

(a)

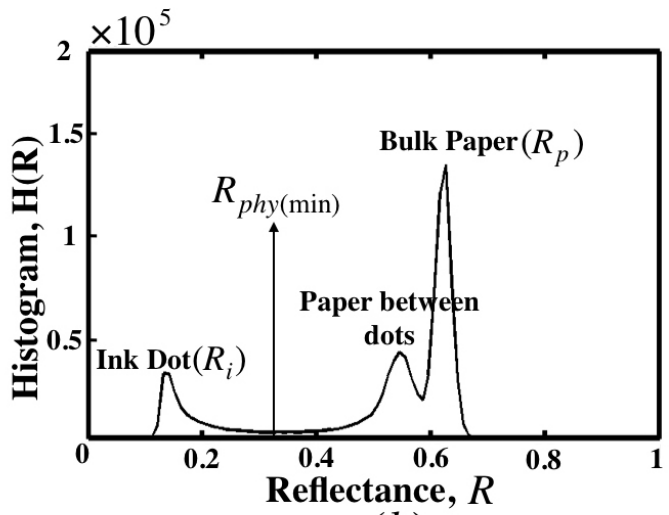

(b)

Figure 5.11: (a) Microscale image including: halftoned area, and unprinted stripe. (b) The reflectance histogram of figure (a).

The proposed microscale image histogram (MIH) approach chooses the $R_{\text {phy(min) }}$, which is the position of the minimum value of the histogram between the two peaks corresponding to the reflectance values of the ink and paper between ink dots. Mathematically, it means that 
this minimum is where the first derivative of the histogram intersects with the reflectance axis while going from negative to positive. This minimum point is chosen to indicate the reflectance at the boundary between dots and paper [ see Figure 5.11 (b)]. The fraction of the physical dot area can be computed from the histogram by Equation (5.9).

$$
a_{e f f,(p h y)}=\frac{\int_{0}^{R_{p h y(m i n)}} H(R) d R}{\int_{0}^{1} H(R) d R} \simeq \frac{\sum_{R=0}^{R_{p h y(m i n)}} H(R)}{\sum_{R=0}^{1} H(R)},
$$

where $H(R)$ denotes the histogram of the reflected image. The physical dot gain, $\Delta a_{p h y}$, as given in Equation (5.10), is the difference between the physical dot area, $a_{e f f,(p h y)}$, and the reference one, $a_{r e f}$,

$$
\Delta a_{p h y}=a_{e f f,(p h y)}-a_{r e f} .
$$

It should be noted that Arney et al. model is mostly suitable for the dots which are halftoned by AM halftoning method. In FM halftoning methods, the dots do not have coherent configuration and it is difficult to find the steepest slope in horizontal or vertical scanning. However, this limitation does not exist in the MIH approach.

Figures 5.12 (a) and (c) show the actual microscale images of two patches with $30 \%$ reference coverage, halftoned by AM and FM halftoning techniques. The patches are printed on the coated paper, $150 \mathrm{gr} / \mathrm{m}^{2}$. By using the MIH approach, the thresholds $R_{p h y(\min )}$ are found for both patches. The physical dot areas are separated and graphically illustrated by using Equation (5.11).

$$
\text { Ink area }= \begin{cases}0 & \text { if } I(x, y)<R_{p h y(\min )} \\ 1 & \text { otherwise }\end{cases}
$$

where $I(x, y)$ indicates the microscale image of halftone print captured by high-resolution camera and the threshold $R_{p h y(\min )}$ is found by using the MIH approach. Figures 5.12 (b) and (d) show the physical dot area (optical dot gain excluded) of the patches halftoned by AM and FM halftoning techniques, shown in Figures 5.12 (a) and (c).

Figure 5.13 shows the histogram of the patches with $30 \%$ reference coverage, halftoned by AM and FM halftoning techniques (see Figures 5.12 (a) and (c)). Although both patches have the same reference coverage $(30 \%)$, the peak for the paper between dots of the patch halftoned by 


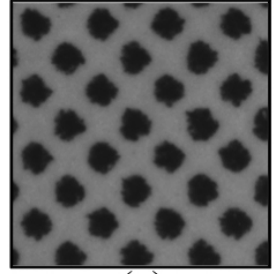

(a)

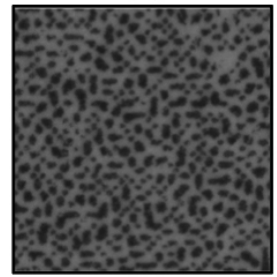

(c)

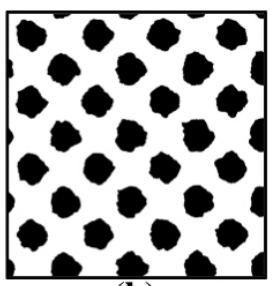

(b)

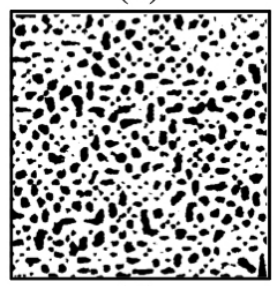

(d)

Figure 5.12: Microscale image and physical dot area of two patches with $30 \%$ reference coverage printed on the coated paper. (a) and (b) Halftoned by AM halftoning technique. (c) and (d) Halftoned by FM (first generation) halftoning technique.

FM has been shifted more to the left compared to the patch halftoned by AM halftoning technique. In other words, the FM halftoning incorporates more optical dot gain compared to the AM halftoning technique. The reason why the FM halftoning has more optical dot gain compared to the AM halftoning is explained in Chapter 7.

Figure 5.14 shows the physical dot gain resulted from the MIH approach and the total dot gain for the patches halftoned by AM halftoning technique from test target 1 (Section 4.4.1). It is observable that the behavior of physical dot gain corresponding to the reference coverage is not symmetrical. For the lighter gray tones, the physical dot gain is very small, while for the darker gray tones the physical dot gain is much bigger. In the dark gray tones, dots have more probability to overlap with their adjacent dots, whereas in the light tones the dots are separated from each other [119]. Ink viscosity and print press pressure are important factors which lead to overlapping dots when the distance between dots is reduced. Hence, the physical dot gain for the dark gray tones is larger than the case where the dots are isolated from each other in the light gray tones. 


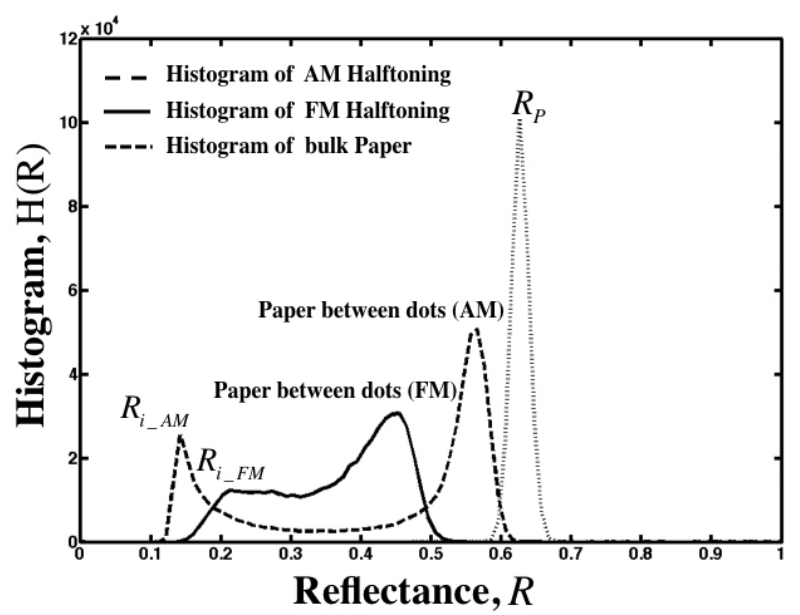

Figure 5.13: The histogram of two patches with $30 \%$ reference coverage, halftoned by AM and FM halftoning techniques.

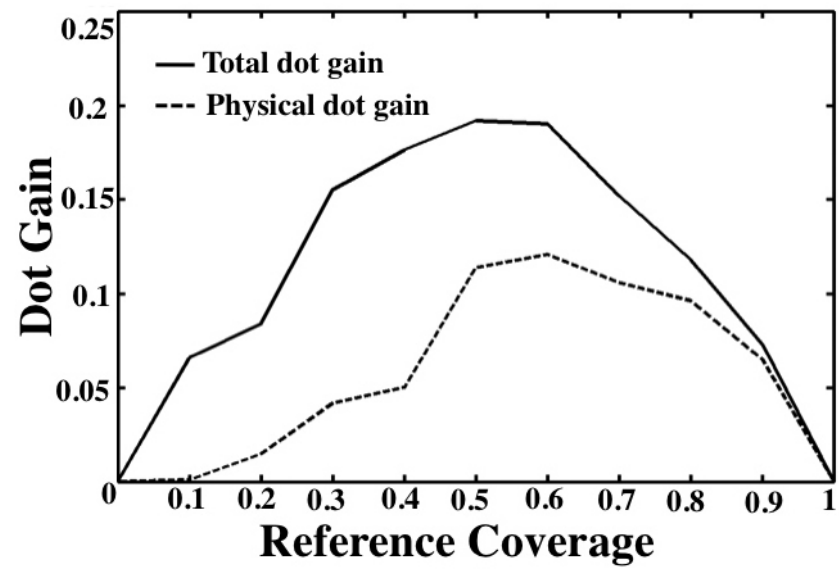

Figure 5.14: Comparison of the total dot gain and the physical dot gain obtained by the MIH approach for the patches halftoned by AM halftoning, using test target 1.

\subsection{Summary and Discussion}

In this chapter three approaches to estimate the total dot gain have been presented and evaluated. One of the approaches is based on the measurements carried out by a spectrophotometer. The two other approaches are based on captured images taken by a high-resolution camera. It has 
been shown that all three approaches produce similar results. By the use of the high-resolution camera the effective dot area has been graphically illustrated. It has also been shown that transmittance incorporates optical dot gain. Moreover, MIH approach based on the reflected microscale image histogram has been proposed to estimate the physical dot area of the halftone print. The MIH approach is generic in the sense that the physical dot gain of any halftone print can be estimated.

This study can help the paper and graphic arts industries to develop their products more efficiently. Testing the approaches on other types of papers and halftoning and color print is an extension to the current study. 


\section{Chapter 6}

\section{MTF Characterization of Paper}

Contents

6.1 Introduction . . . . . . . . . . . . 89

6.2 Reflection Image Model . . . . . . . . . 90

6.3 MTF Characterization by MIH Approach . 91

6.4 MTF Measured by Knife-Edge Method . . . 95

6.5 MTF Simulated by Monte-Carlo . . . . . . 96

6.6 MTF Study of Microscale Transmitted Images 97

6.7 Validity Evaluation of MTF Models . . . . 98

6.8 Summary and Discussion . . . . . . . . . 101 


\subsection{Introduction}

Light scattering in the paper has a significant influence on image quality of the halftone prints. The light scattering effect in the paper can be quantified by Modulation Transfer Function (MTF) of the paper, which is the Fourier transform of the PSF (Point Spread Function). Generally the PSF is the optical impulse response of paper. Much research has been reported on methods to measure and simulate the MTF of paper. Although measuring the MTF is a complicated task many methods have been proposed in the literatures.

The knife-edge method proposed by Yule and Nielsen have been well established over the past decades [121]. The method is based on measuring the Line Spread Function, LSF, of paper from the Edge Spread Function, ESF, obtained by the sharp knife-edge projected on the paper.

In 1997, Inoue et al. proposed a method to project sinusoidal test patterns to the paper's surface [53]. The MTF of paper was then obtained by measuring the reflection density distribution of the projected sinusoidal test pattern by using a microdensitometer. This method directly measures the MTF of the paper. It should be noted that to obtain more accurate results, it requires to project and measure a lot of sinusoidal test patterns with different spatial frequencies.

In 1998, Inoue et al. proposed another method to measure the MTF. In this method a sinusoidal test pattern printed on film is contacted onto the paper [54]. This method is experimentally simpler than the projecting method but still needs to iteratively measure several test patterns.

In 1998, Rogers proposed a series-expansion bar-target technique to measure the paper's MTF [99]. The MTF is obtained by projecting the bar-target on the paper and measuring the response. In this method, bar-target image data are expanded into a Fourier series, and the paper's MTF is given by the series-expansion coefficients. Although it is not simple to create an ideal bar-target image with the sharp edges, the method is experimentally efficient since only one bar-target image needs to be measured.

Another direct method for measuring the MTF of paper is proposed by Ukishima [110]. The MTF is measured from the ratio in spatial frequency domain between the responses of incident pencil light to the paper and the perfect specular reflector.

Several indirect methods have also been proposed to estimate the 
MTF of paper; based on Kubelka-Munk theory [7,27], modeling the Yule-Nielsen effect by the contacted high-resolution halftone line screen on the paper [3], and based on Monte-Carlo simulation [19].

In Chapter 5, the Microscale Image Histogram (MIH) approach was proposed to separate the physical dot area of ink from the paper. Having the physical dot area makes it possible to separate the effect of the optical dot gain and thereby to estimate the MTF of the substrate. In this chapter, to validate the MIH approach, the MTFs of eleven paper samples of test target 3, described in Section 4.4.3, are simulated and compared with the measured MTF by knife-edge method and the MTF obtained by MC simulation. Moreover, in this chapter we confirm that using the transmitted images to estimate the physical dot gain is not an appropriate approach. The study in this chapter has been published in [78].

\subsection{Reflection Image Model}

In Section 3.3.2 a model for the reflectance of the halftone print based on the ink layer transmittance, point spread function of the paper and the reflectance of the paper was described in Equation (3.4). Figure 6.1 illustrates the light transfer behavior in the halftone print. We consider the halftone printed paper as being made of two layers; the ink layer made of ink dots and voids, and the paper substrate layer. The light transfer behavior can be explained as follows,

1. The incident light illuminates the ink layer.

2. The light transmits through the ink layer whose transmittance is denoted by $\mathrm{T}(\mathrm{x}, \mathrm{y})$.

3. The transmitted light enters the paper.

4. The light is scattered in paper by $\operatorname{PSF}_{\mathrm{p}}(x, y)$ and reflected back from the paper by $R_{\mathrm{p}}$.

5. The reflected light transmits again through the ink layer.

The Fourier transform of $\operatorname{PSF}_{\mathrm{p}}(x, y)$ is called the optical transfer function $(\mathrm{OTF})$. The OTF is separated to its absolute value and phase shift, $\exp \{j \theta(u, v)\}$. In case of $\exp \{j \theta(u, v)\}=1$ (system has no shift), the PSF has the spatial isotropic property and the absolute value of OTF 


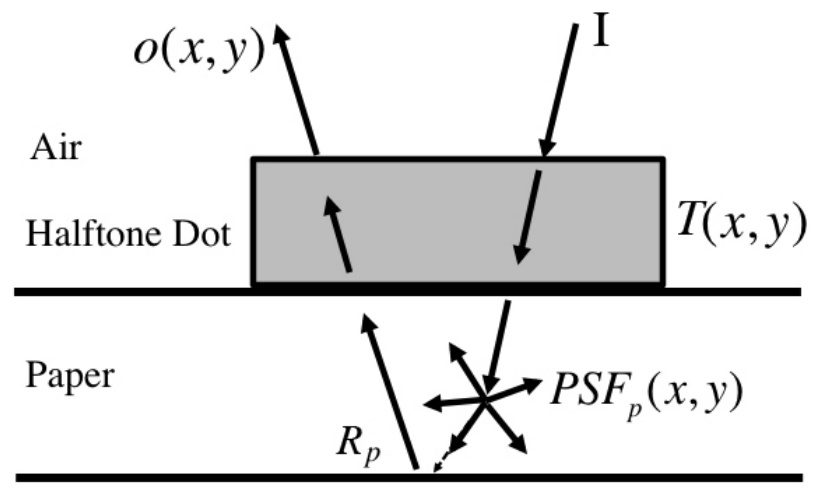

Figure 6.1: Light transfer behavior in the reflection image model.

is alternated to $\operatorname{MTF}_{\mathrm{p}}(u, v)$. Equation (3.4) can now be expressed by using the MTF of the paper in the Fourier domain.

$$
R(x, y)=\mathcal{F}^{-1}\left[\mathcal{F}\{T(x, y)\} \cdot \operatorname{MTF}_{\mathrm{p}}(u, v)\right] \cdot T(x, y) R_{\mathrm{p}}
$$

where $\mathcal{F}$ and $\mathcal{F}^{-1}$ denote the Fourier transform and the inverse Fourier transform, respectively. In this model, the reflectance $R(x, y)$ is affected by both physical and optical dot gain, while $T(x, y)$ is only affected by the physical dot gain. The optical dot gain effect is expressed inside the $\operatorname{MTF}_{\mathrm{p}}(u, v)$ function. Therefore, the reflection image model would be suitable for separately analyzing the physical and optical dot gain.

\subsection{MTF Characterization by MIH Approach}

The proposed MIH approach in this dissertation is used to find $R_{p h y(\min )}$ to separate the physical dot area from the paper. Once the physical dot gain is separated from the optical dot gain, it is possible to estimate the MTF through the reflection image model, Equation (6.1). The spatial distribution of transmittance of the ink layer $T(x, y)$ is given by,

$$
T(x, y)= \begin{cases}0 & \text { if } \quad I(x, y)<R_{\text {phy }(\min )} \\ 1 & \text { otherwise, }\end{cases}
$$

where $I(x, y)$ is the microscale image of the halftoned area, and the threshold $R_{\text {phy }(\min )}$ is found by using the MIH approach. It has to be 
pointed out here that $T(x, y)$ represents the actual physical dot shape after print [ see Figure 5.12 (b)]. This image can be useful for the study of the ink spreading behavior by for example comparing this image with its corresponding digital bitmap.

In order to estimate the MTF of the paper, three functions proposed in references $[53,99,109]$ are used. These are,

$$
\begin{gathered}
\operatorname{MTF}_{1 \mathrm{p}}(u, v)=\left[1+\left(2 \pi d_{1}\right)^{2} \cdot\left(u^{2}+v^{2}\right)\right]^{\frac{-3}{2}}, \\
\operatorname{MTF}_{2 \mathrm{p}}(u, v)=\left[1+\left(2 \pi d_{2}\right)^{2} \cdot\left(u^{2}+v^{2}\right)\right]^{\frac{-1}{2}}, \\
\operatorname{MTF}_{3 \mathrm{p}}(u, v)=e^{\frac{-d_{3}^{2} \cdot\left(u^{2}+v^{2}\right)}{2}},
\end{gathered}
$$

where $u$ and $v$ are the spatial frequency (cycle/mm) and $d_{1}, d_{2}, d_{3}$ are fitting coefficients [78]. Equations (6.3 - 6.5) are used separately in Equation (6.1) to simulate $R(x, y)$ for the given $T(x, y)$ estimated with the MIH approach. The fitting coefficients, $d_{1}, d_{2}, d_{3}$, are determined by minimizing the difference between the measured and simulated effective coverage. The effective coverage can be approximated by Murray-Davies equation,

$$
a_{e f f}=\frac{R_{\mathrm{ave}}-R_{\mathrm{p}}}{R_{\mathrm{i}}-R_{\mathrm{p}}}
$$

The value of $R_{\text {ave }}$ for the measured and simulated effective coverage is obtained differently. For the measured effective coverage, $R_{\text {ave }}$ is the average value of the halftone patch, while for the simulated case, $R_{\text {ave }}$ is the average value of $R(x, y)$ in Equation (6.1). In the measured effective coverage, $R_{\mathrm{i}}$ and $R_{\mathrm{p}}$ are the reflectance of the full tone ink and paper, respectively. In the simulation, due to the binary characteristic of $T(x, y)$ in Equation (6.2), $R_{\mathrm{i}}$ is 0 and $R_{\mathrm{p}}$ is 1 . The flow chart of the iterative algorithm shown in Figure 6.2 illustrates how to estimate the paper's MTF by optimizing the fitting coefficient, $d$.

Figure 6.3 (a) shows the MTF curves obtained by using Equations $\left(6.3\right.$ - 6.5) for a $150 \mathrm{gr} / \mathrm{m}^{2}$ paper sample from the test target 3. Figure $6.3(\mathrm{~b})$ shows the simulated total dot gain obtained by fitting three 


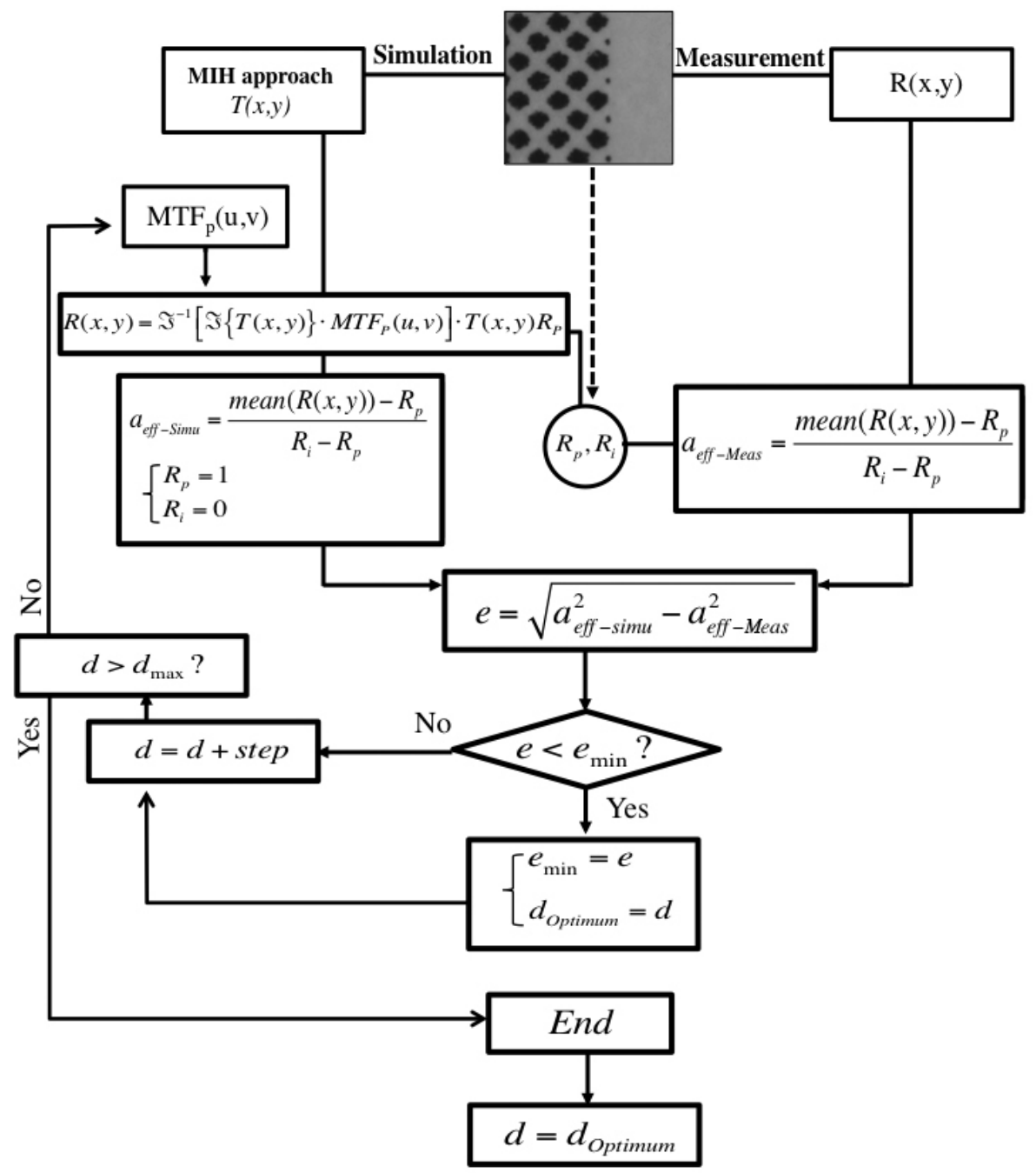

Figure 6.2: Flow chart of the iterative algorithm to estimate the MTF of paper.

different MTFs and the measured total dot gain, which result in identical dot gain curves. Although the shape of the MTF curves in Figure 6.3 (a) differ at higher frequencies, the inverse of the frequency at the half maximum of MTF, representing $k_{p}$, are very close to each other. The reason that these curves give almost identical dot gain despite their differences at higher frequencies, is that the optimization of the param- 
eters $d_{1}, d_{2}, d_{3}$ in Equations (6.3 - 6.5) are based on the average value of the microscale images. Therefore, these differences at higher frequencies have no impact on the average of $R(x, y)$. Hence, the $k_{p}$ is insensitive to the shape of MTF and it is not appropriate to be considered as a single metric for MTF.
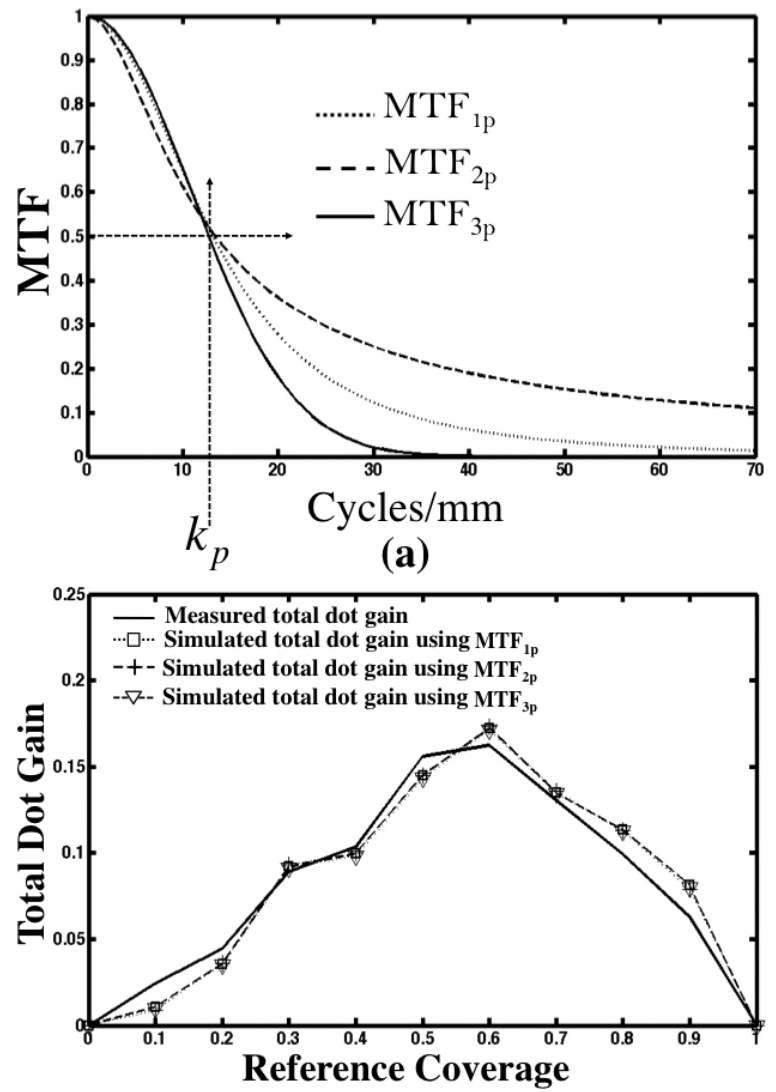

(b)

Figure 6.3: (a) The MTF curves obtained by using Equations (6.3 - 6.5) for a $150 \mathrm{gr} / \mathrm{m}^{2}$ paper sample, $k_{p}$ represents the inverse of the frequency at the half maximum of MTF. (b) Comparison of the simulated total dot gain obtained by fitting three different MTFs function and the measured total dot gain. 


\subsection{MTF Measured by Knife-Edge Method}

In this section the knife-edge method reported by Yule et al. [121] is used to directly measure the paper's MTF. In this method a high precision knife-edge pattern on high-resolution multifunction target is contacted to the paper surface [96]. With the high-resolution camera, the spatial resolved reflectance from the edge is measured and averaged along the edge.

Figure 6.4 (a) shows the image of knife-edge pattern projected on paper and captured by the high-resolution camera for the $150 \mathrm{gr} / \mathrm{m}^{2}$ paper sample. The edge information generated from a line scan through the image is represented by the edge spread function, $\operatorname{ESF}(x)$. In this figure, the marked points (a, b, c, $\mathbf{d}$, and e) along the line passing through the edge show the locations which correspond to the positions at the ESF curve in Figure 6.4 (b). The marked points indicate the intensity of the reflectance through the knife edge, that is gradually increased from left to right (i.e. black to white). For instance, the points "a" and "e" show the minimum and maximum reflectance, which are related to the black and white area of the image in Figure 6.4 (a). The point "c", which is located on the edge, indicates the steepest slope of the ESF curve.

The first derivative of the $E S F(x)$ results in a line spread function, $\operatorname{LSF}(x)$, see Equation (6.7). It provides a direct measure of the lateral distance light, which is scattered from the illuminated edge [3]. Figure 6.4 (b) shows the edge spread function $E S F(x)$ of the $150 \mathrm{gr} / \mathrm{m}^{2}$ paper sample.

$$
L S F(x)=\frac{d}{d x} \operatorname{ESF}(x)
$$

Taking the Fourier transform of the line spread function, $\mathcal{F}(L S F(x))$, gives a complex function in terms of frequency $(\omega)$. The magnitude of the Fourier transform of $\operatorname{LSF}(x)$, is defined as the paper's MTF.

$$
\operatorname{MTF}(\omega)=\left|\int_{-\infty}^{+\infty} \operatorname{LSF}(x) e^{-j 2 \pi \omega x} d x\right|
$$

Figure 6.4 (c) illustrates the MTF of the paper $\left(150 \mathrm{gr} / \mathrm{m}^{2}\right)$, measured by the knife-edge method. The knife-edge method is experimentally efficient because only one projected edge image needs to be mea- 
sured. However, the measurement accuracy is not significant since the noise in the knife-edge response is increased when the derivation is calculated to obtain the LSF from the ESF.

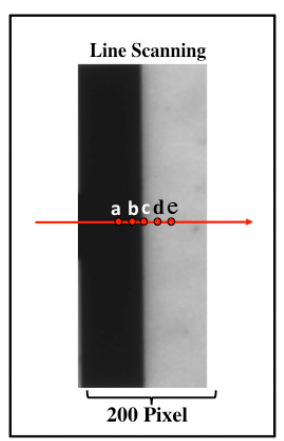

Knife Edge

(a)

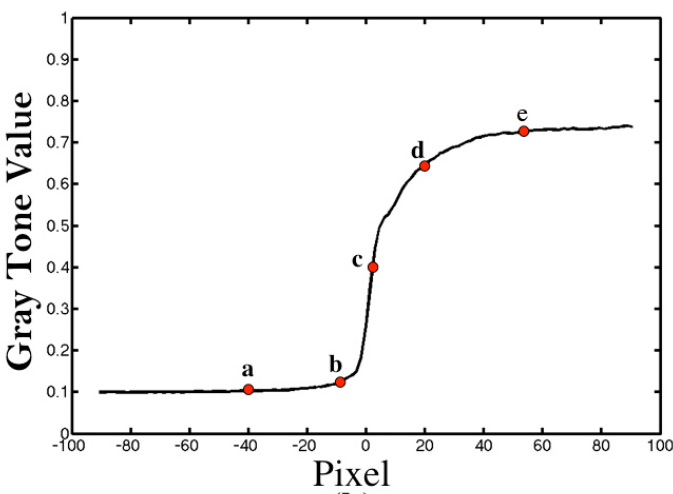

(b)

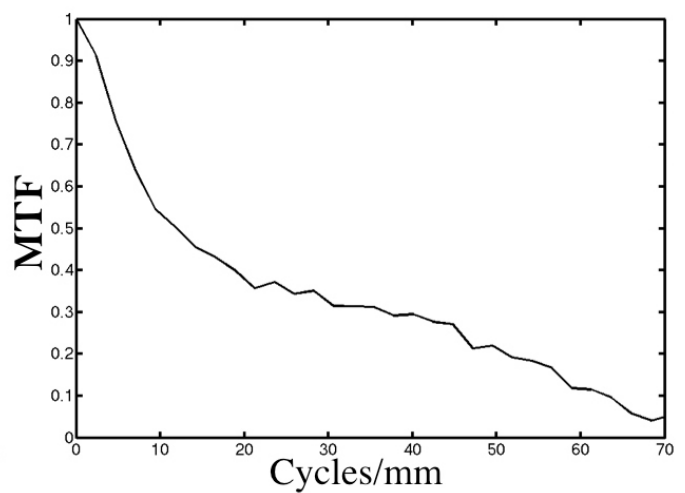

(c)

Figure 6.4: (a) The image of contacted knife-edge pattern for the $150 \mathrm{gr} / \mathrm{m}^{2}$ paper sample. (b) ESF (edge spread function) computed by scanning the line through the contacted knife-edge pattern. (c) MTF obtained by knife-edge method.

\subsection{MTF Simulated by Monte-Carlo}

In Section 3.4.8, the model of photon transfer in a medium based on Monte-Carlo simulations was presented. In this section in order to calculate the MTF of paper, the Kubelka-Munk scattering, $S$, and absorption, $K$, coefficients of the unprinted papers are determined from the 
measured reflectance factors using the Kubelka-Munk theory [63]. In the general radiative transfer (RT) theory [55], scattering coefficient, $\sigma_{s}$, and absorption coefficient, $\sigma_{a}$, are approximated with the so-called similarity relations $[71,72]$. For the case of isotropic single scattering, the similarity relations are $\sigma_{s}=\frac{4}{3} S$ and $\sigma_{k}=2 K$. The Monte-Carlo software Open PaperOpt is used to compute the ESF of the simulated substrates [18]. A beam incident at $45^{\circ}$ illuminates half of a $500 \mu \mathrm{m} \times$ $500 \mu \mathrm{m}$ surface and the spatially resolved reflectance is simulated with a resolution of $1 \mu \mathrm{m}$. The simulated spatially resolved reflectance is cut to a $400 \mu m \times 400 \mu m$ area to avoid edge effects in the Monte-Carlo simulation and then averaged along the illumination edge and normalized to obtain the one-dimensional edge response. As for the measured ESF, the derivative of the edge response is then Fourier transformed to obtain the MTF.

The aforementioned Monte-Carlo simulation study of MTF was done at Mid Sweden University ${ }^{1}$ as a collaboration work for completing the performance evaluation of the author's MTF study, and hence the MC simulation results is not considered as the contribution of this dissertation.

\subsection{MTF Study of Microscale Transmitted Im- ages}

In Section 5.2.1, it has been shown that although the transmitted image has less optical dot gain compared to the reflected image, the transmittance also incorporates optical dot gain. However, it has been claimed in some literature that the transmitted images only include the physical dot gain effect $[61,116]$. They have proposed that $T(x, y)$ can be measured with a transparent optical microscope. They used a microscope with two light sources to illuminate the light from above and below the paper. Here, if it is assumed that the transmitted images are not affected by optical dot gain, the ink layer transmittance $T(x, y)$ in Equation (6.2) can be replaced with,

$$
T(x, y)=\left\{\begin{array}{lll}
0 & \text { if } \quad I_{t}(x, y)<R_{t} \\
1 & \text { otherwise }
\end{array}\right.
$$

\footnotetext{
${ }^{1}$ Ludovic G. Coppel, Department of Natural Sciences, Engineering and Mathematics, Mid Sweden University.
} 
where $I_{t}(x, y)$ is the transmitted microscale image of the halftoned area. Equation (6.9) shows that the ink layer transmittance $T(x, y)$ varies by the selection of the threshold value $R_{t}$. Hence, $R_{t}$ is chosen with the objective to minimize the difference between the average of $T(x, y)$, and the measured effective coverage of the transmitted image. By substituting $T(x, y)$ obtained from the transmitted image in Equation (6.1) and minimizing the difference between the measured and the simulated effective coverage, MTF is found.

\subsection{Validity Evaluation of MTF Models}

The test target 3, described in Section 4.4.3 with different reference coverages of gray $(0,10,20, \ldots, 90,100 \%)$ printed on 11 different coated papers with basis weight ranging from $65 \mathrm{~g} / \mathrm{m}^{2}$ to $150 \mathrm{~g} / \mathrm{m}^{2}$ are used. All three MTF function Equations $(6.3-6.5)$ are placed individually in Equation (6.1). By minimizing the root mean square difference ( $\Delta \mathrm{RMS})$ between the measured and the estimated effective coverage, the best fitting coefficients $\left(d_{1}, d_{2}, d_{3}\right)$ for all eleven paper samples are found [Table (6.1)].

Table 6.1: Fitting coefficients obtained from three MTF functions for eleven coated papers. The $k_{p}$ values calculated by MIH and Monte-Carlo simulations.

\begin{tabular}{cl|ccc|cc}
\hline Samples & $g r / m^{2}$ & $d_{1}$ & $d_{2}$ & $d_{3}$ & $M I H\left(k_{p}\right)$ & $M C\left(k_{p}\right)$ \\
\hline PF02 & 65 & 0.009 & 0.020 & 0.092 & 0.0878 & 0.0921 \\
PF04 & 70 & 0.009 & 0.020 & 0.092 & 0.0878 & 0.0893 \\
PF09 & 70 & 0.012 & 0.026 & 0.121 & 0.0943 & 0.0918 \\
PF13 & 80 & 0.018 & 0.041 & 0.186 & 0.1322 & 0.1289 \\
PF18 & 80 & 0.012 & 0.026 & 0.121 & 0.0924 & 0.0950 \\
PF19 & 80 & 0.010 & 0.022 & 0.103 & 0.0873 & 0.0926 \\
PF23 & 115 & 0.013 & 0.030 & 0.139 & 0.1172 & 0.1198 \\
PF24 & 115 & 0.011 & 0.025 & 0.116 & 0.0978 & 0.0920 \\
PF31 & 115 & 0.009 & 0.022 & 0.099 & 0.0941 & 0.0934 \\
PF33 & 150 & 0.012 & 0.026 & 0.121 & 0.1119 & 0.1125 \\
PF35 & 150 & 0.011 & 0.025 & 0.116 & 0.0978 & 0.1048 \\
\hline
\end{tabular}


Figure 6.5 (a) compares the estimated MTF obtained by MIH approach using the three MTF functions , Equations (6.3 - 6.5), to the MTF obtained by the knife-edge method for a $150 \mathrm{~g} / \mathrm{m}^{2}$ sample. This figure clearly illustrates that $\mathrm{MTF}_{2 \mathrm{p}}$ better represents the measured MTF. It is worth noting that Equations (6.3 - 6.5) lead to similar frequencies at half MTF maximum, but the shapes are very different.
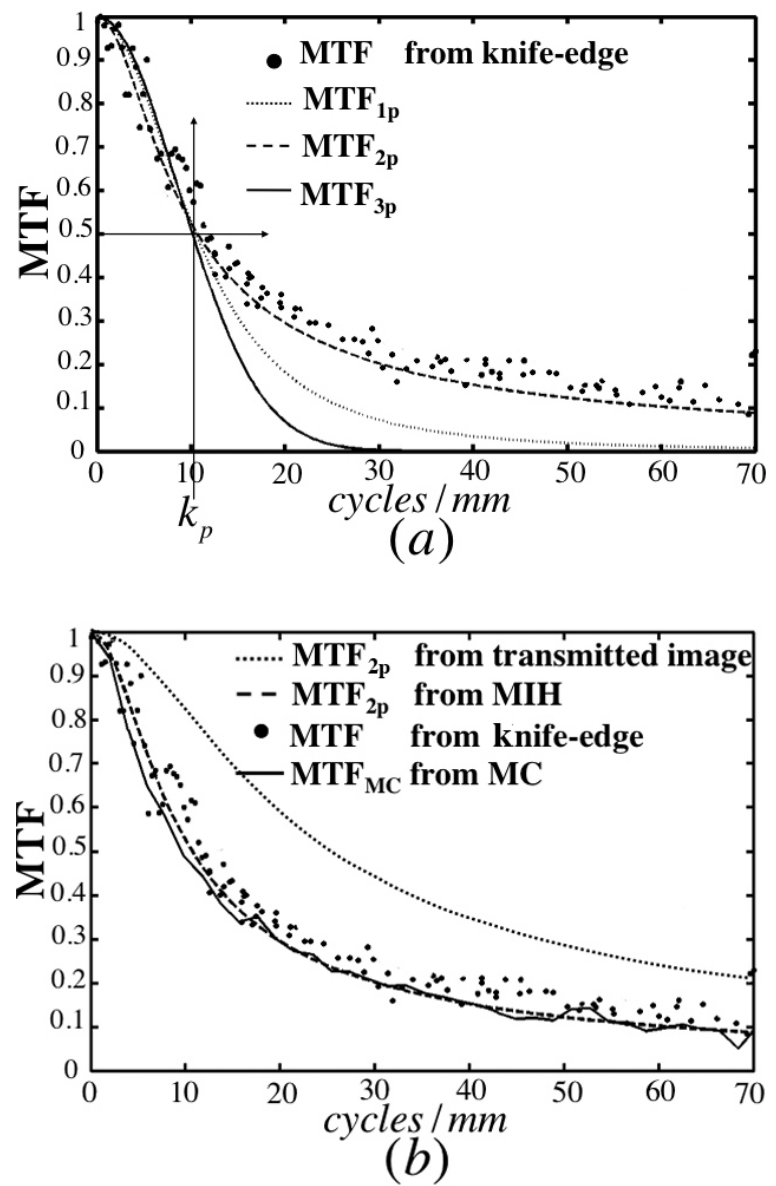

Figure 6.5: (a) Comparison of the three MTF curves obtained by MIH and the knife-edge method for a $150 \mathrm{gr} / \mathrm{m}^{2}$ sample. (b) Comparison of the MTFs obtained by MIH (dashed line), Monte-Carlo simulations (solid line), knife-edge method (dot line), and transmitted images (small dashed line). 
Figure 6.5 (b) compares the MTF obtained using MIH approach to the MTF obtained with the knife-edge method, and Monte-Carlo simulations and the MTF obtained using transmitted images. It is obvious that the MTF obtained with MIH, Monte-Carlo and knife-edge methods produce similar results while $\mathrm{MTF}_{2 \mathrm{p}}$ obtained from transmitted image is significantly wider. This is valid for all 11 samples in this study. Wider MTF means less lateral light scattering and thus less optical dot gain. In case of no optical dot gain, the paper's MTF would be parallel to the $\mathrm{X}$-axis. Hence it is obvious from Figure 6.5 (b) that the transmitted images also incorporate optical dot gain, which confirms the findings in Section 5.2.1 [75].

Figure 6.6 shows the inverse half-frequency at half MTF maximum $\left(k_{p}\right)$ of eleven paper samples estimated with the MIH approach versus $k_{p}$ obtained with Monte-Carlo simulations. It should be noted that the MTFs estimated with the MIH approach are the MTF of the printed papers while Monte-Carlo simulations are based on the optical properties of the unprinted papers. Ink and ink carrier penetration into the substrate may affect the scattering properties of the substrate. However the linear regression between $k_{p}$ values obtained with Monte-Carlo and MIH concludes that the proposed MIH approach successfully separates physical and optical dot gain.

The $k_{p}$ values of 11 paper samples which are calculated by MIH and Monte-Carlo simulations are reported in Table (6.1) and the results has a maximum difference of $0.7 \%$.

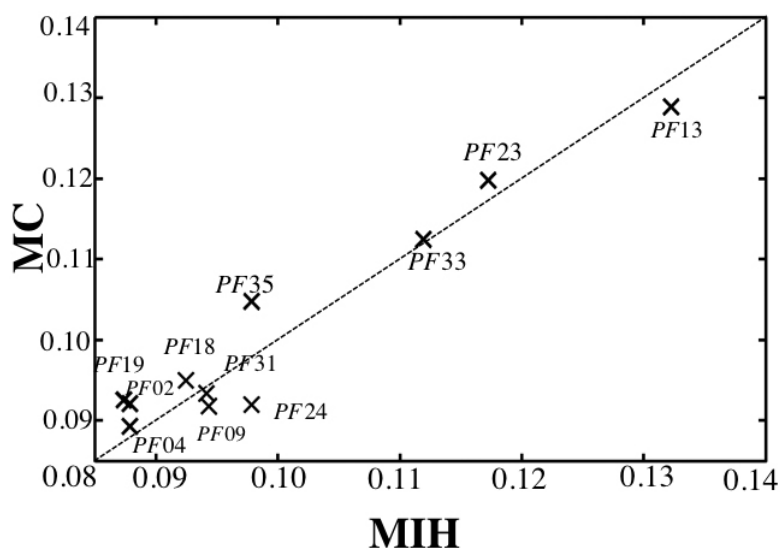

Figure 6.6: Comparison of $k_{p}$ values of 11 samples estimated with MIH approach versus Monte-Carlo simulations. 


\subsection{Summary and Discussion}

In Chapter 5, the MIH approach based on the reflected microscale image histogram was proposed to estimate the physical dot area of the halftone print. In this chapter to validate the mentioned approach, the MTF of eleven paper samples were simulated and compared with measured MTF by knife-edge method and the MTF obtained with Monte-Carlo simulation. The results showed that the proposed microscale image histogram (MIH) approach successfully estimates the physical dot area of halftone print. The MIH approach is generic in the sense that the physical dot gain of any halftone print can be estimated. Separately monitoring physical and optical dot gain can also help the paper and graphic art industries to characterize ink dot placement, ink spreading on the paper and optical dot gain effects. 


\section{Chapter 7}

\section{Characterizing Dot Gain for Different Dot Shapes}

Contents

7.1 Introduction . . . . . . . . . . . 105

7.2 Comparison of Optical Dot Gain for Different Dot Shapes . . . . . . . . . . . . 105

7.3 Ink and Paper Interaction . . . . . . . . 107

7.4 Dot Gain and Halftoning Techniques . . . . 111

7.5 Color Gamut Determination . . . . . . . . 117

7.5.1 Gamut Volume . . . . . . . . . . . . . . . . 119

7.6 Summary and Discussion . . . . . . . . 119 


\subsection{Introduction}

In previous works the physical and optical dot gains were mostly analyzed by illustrating their respective dot gain curves $[30,45,119]$. These curves only show the relationship between the effective dot area and the reference (or nominal) dot area, and do not illustrate the shape of the printed dots including the effect of optical dot gain, which is closely related to the optical properties of the paper and physical dot shape. Different paper substrates might have different scattering properties. In Chapter 5, the MIH approach based on the reflected microscale image histogram was proposed to estimate the physical dot area of halftone prints. Since the physical dot shape can be separated, it is possible to simulate the optical dot gain effect for different dot shapes used in different halftoning techniques by using a proper MTF model.

In Chapter 6, three different exponential functions were suggested to estimate the MTF of paper $[53,99,109]$. It was shown that among different functions, the model, which was proposed by Rogers [99], gave a better fit to the MTF obtained with the knife-edge method [121], and MTF simulated by Monte-Carlo simulation [18]. Hence, the study in this chapter is based on the $\mathrm{MTF}_{2 \mathrm{p}}$ model previously presented in Equation (6.4).

The study of this chapter includes the performance evaluation of the FM2nd halftoning technique in terms of optical dot gain, ink behavior, and color gamut compared to AM and FM first generation halftoning techniques. All the investigations have been applied and compared for two types of paper: coated and uncoated. In this chapter, first the relation between dot shape and optical dot gain is characterized, then the investigation of ink behavior is presented, followed by color gamut illustration. The research presented in this chapter has been previously presented in $[79,80]$.

\subsection{Comparison of Optical Dot Gain for Differ- ent Dot Shapes}

In Chapter 3, three different halftoning techniques AM, FM (FM first generation), and FM2nd (FM second generation) were presented. It was shown that the dots created by using these techniques have different shapes. It has been previously shown that both physical and optical 
dot gain increase with higher print resolution, and thus smaller dot size [90]. To investigate the dependency of optical dot gain on the dot shape, the optical dot gain is simulated for dots with different shapes and perimeters but with the same area. For this purpose, six dots with different shapes are considered for investigation. Figure 7.1 shows the six dot shapes which have different perimeters but with the same area. The reference coverage of all samples is around 10\%. In Figure 7.1, the first two dot shapes are the basic AM round (or circular) and square. The dot shapes No. 3, 4 and 5 are chosen from FM2nd halftoning technique. Dot shape No. 6 is a collection of FM dots, in which the sum of their areas is equal to the areas of the other shapes in Figure 7.1. The numbering of the dot shapes is based on the size of their perimeters, i.e. the first dot shape has the minimum perimeter and the sixth dot shape has the maximum perimeter among all.

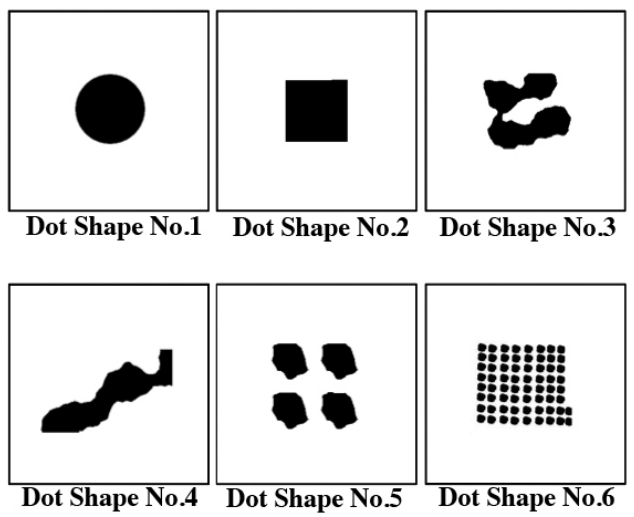

Figure 7.1: The six different dot shapes; No.1 and 2 dot shapes from AM, No. 3, 4 and 5 from FM second generation and No. 6 from FM first generation.

By using Equations (7.1 - 7.3), proposed in Chapter 6, the optical dot gain is simulated for all six samples.

$$
\begin{gathered}
\operatorname{MTF}_{2 \mathrm{p}}(u, v)=\left[1+\left(2 \pi d_{2}\right)^{2} \cdot\left(u^{2}+v^{2}\right)\right]^{\frac{-1}{2}} \\
R(x, y)=\mathcal{F}^{-1}\left[\mathcal{F}\{T(x, y)\} \cdot \operatorname{MTF}_{2 \mathrm{p}}(u, v)\right] \cdot T(x, y) R_{\mathrm{p}} \\
a_{\text {opt }}=\text { mean }(R(x, y))-\text { mean }(T(x, y))
\end{gathered}
$$


In Equation (7.1), the fitting coefficient is set to $d_{2}=0.29$, which is computed for the uncoated paper $150 \mathrm{gr} / \mathrm{m}^{2}$. The image of each dot shape in Figure 7.1 is used as $T(x, y)$ in Equation (7.2). Comparing the optical dot gain for different dot shapes shows the dependency of optical dot gain on the dot shape perimeter. As can be seen in Figure 7.2, the optical dot gain increases when the perimeter of dot shape increases. However, one can notice from Figure 7.2 that there is a limit to the ratio of perimeter to area at which the optical dot gain is saturated. It means that above a certain value of the dot perimeter, no matter how much the perimeter is increased, the value of the optical dot gain will not be increased [79]. It is observable that the optical dot gain is dependent on the dot shape and therefore it can be concluded that the amount of optical dot gain is different for these three types of halftoning techniques. AM has the minimum optical dot gain and FM (first generation) has the maximum optical dot gain when printed on the same paper.

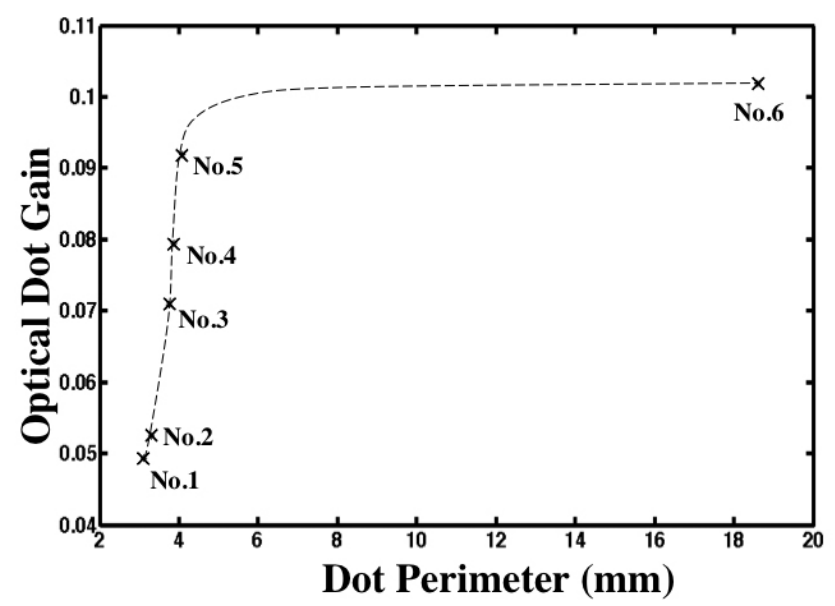

Figure 7.2: Comparison of optical dot gain for different dot shapes on the uncoated paper $(d=0.29)$.

\subsection{Ink and Paper Interaction}

A number of important factors to be considered in all printing methods, are the mechanism of ink penetration, the morphology of the finally settled ink and its optical properties. The final ink setting is dependent on the chemical and physical interaction between ink and paper. The 
paper properties such as smoothness and porosity have an impact on printing ink performance. These properties promote printing ink penetration or spreading which causes loss or gain of print density [60]. The ink spreads and sets in different ways. The printed ink goes into the paper and spreads sideways, which increases the dot diameter. Due to the inhomogeneous pore structure of paper, the ink distribution on the rough surface of uncoated paper is complex and not well defined.

In offset printing, most of the physical dot gain occurs before the ink touches the paper surface, hence variation of papers have less impact on the physical dot gain [52]. However in flexo and inkjet printing, the surface tension and penetration of the paper surface could cause the ink to spread more or less on the surface or inside the coating layers. The mechanical action between cylinders has also an impact on the ink spreading in offset printing. Cylinder pressure causes more compression and deformation on softer paper surface. In this case for the uncoated paper the ink spreads more through the pores of the paper and makes a non-uniformed dot shape [37].
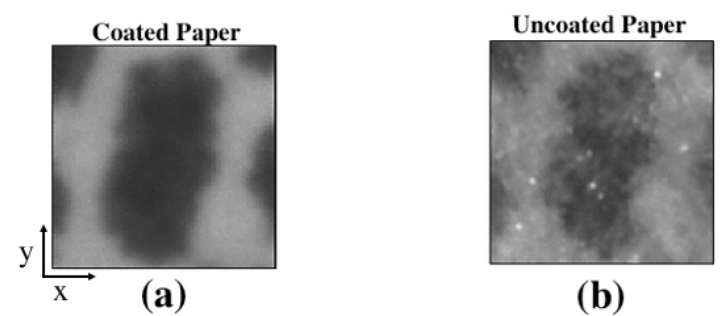

(b)

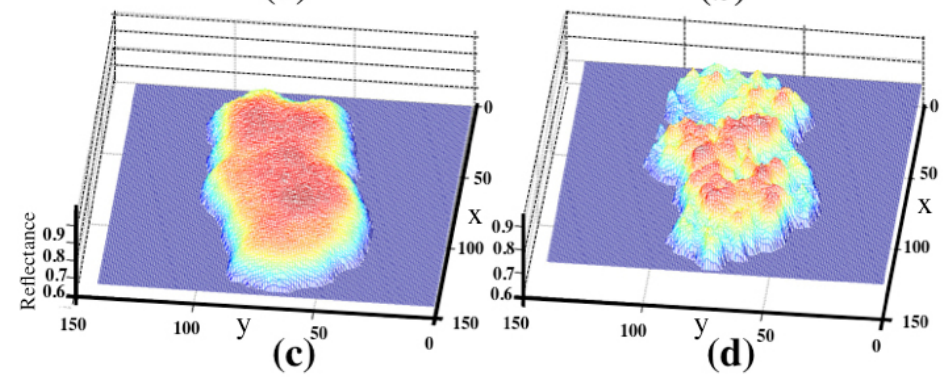

Figure 7.3: (a) and (b) The microscale image of a dot printed on coated and uncoated paper. (c) and (d) The three-dimensional illustration of the dot printed on coated and uncoated paper.

Figure 7.3 shows the microscale image and three-dimensional illustration of a dot with the same original dot shape, printed on both coated and uncoated paper. The physical dot areas are separated from the pa- 
per by applying the MIH approach to the microscale images. The threedimensional shape of the dots are illustrated based on the reflectance value of each pixel. In other words, the red area shown in Figure 7.3 (c) and (d) has lower reflectance value compared to the other areas. It should be noted that, due to the micro-glossy agents, the white spots shown in the uncoated paper, Figure 7.3 (b), are also considered as high reflectance value. However by rotating the illumination angle or rotating the XY-plate these white spots are also moved.

Due to the paper's structure, the dots get different formation. The coated paper has a uniform surface and most of the ink spreads on surface of the paper. Hence the dots printed on the coated paper have a homogeneous shape.

In the uncoated paper, more ink penetrates into the pore of the paper, hence the surface of the dot is not uniform. Figure 7.3 (d) shows the three-dimensional shape of the dot printed on the uncoated paper and as it can be seen it looks like a mountain and valley.

Therefore, the density of the color inks are dependent on the surface properties of the chosen paper; smoothness, porosity, gloss, etc. The higher the roughness and porosity value, the higher the ink demand. For the same amount of ink, the paper with a higher roughness and porosity, has a lower print density [111]. Therefore, the same amount of ink will cover less area on the uncoated paper compared to the coated one, and the density of ink is less for the uncoated paper.

In this chapter, in order to investigate the ink behavior of the offset print press, the dots with the same original shape in the digital bitmap are printed on both coated and uncoated papers. The MIH approach is used to estimate the physical ink area. Figure 7.4 shows four examples of printed dots with the same original shape printed on both coated and uncoated paper. The physical dot area for all four dots are separated. In Figure 7.4 the separated dots are placed on top of each other. The dots are selected from FM2nd halftoning with different sizes. In Figure 7.4 the yellow part shows the area which is common between the coated and uncoated paper. The red area belongs to the dot printed on the coated paper and the green area belongs to the dot printed on the uncoated paper.

Table 7.1 illustrates the relation between the type of paper and the dot gains which are estimated from the dots in Figure 7.4. Table 7.1 shows that the total dot gain is larger for the dots printed on the uncoated paper. However, when the physical dot gain is separated, it is 


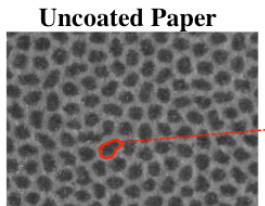

Coated Paper

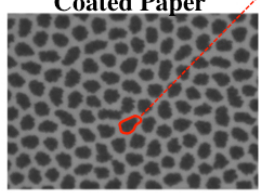

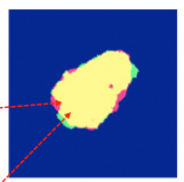

(a)

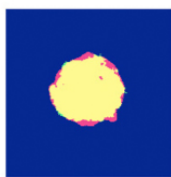

(c)

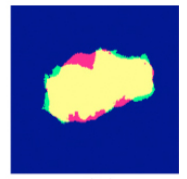

(b)

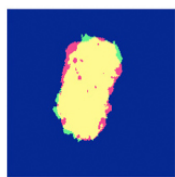

(d)

Figure 7.4: Comparison of dots with the same original shape printed on both coated and uncoated paper.

found to be larger for the coated paper. Microscopic images of the prints also reveal that the ink penetrates into the pores and cavities of the uncoated paper, resulting in inhomogeneous dot shapes. For the coated paper, the ink spreads on top of the surface, giving a more homogenous dot shape, but also covering a larger area, and hence larger physical dot gain. Now the question is why the total dot gain of uncoated paper is larger than the coated paper in the offset printing?

The optical dot gain causes the total dot gain to become larger on the uncoated paper. It has been presented in Chapter 6, that the optical dot gain is dependent on the MTF of paper [78]. In the previous section, it has also been shown that the optical dot gain is dependent on the dot perimeter and it is larger for bigger dot perimeters [79].

Table 7.1: Comparison of total, physical and optical dot gains of dots in Figure 7.4 .

\begin{tabular}{|c|c|ccc|c|}
\hline Sample & Paper Type & \multicolumn{3}{|c|}{ Dot Gain } & Perimeter \\
& & Total & Physical & Optical & $(\mathrm{mm})$ \\
\hline $\mathrm{a}$ & Coated & 0.074 & 0.066 & 0.008 & 0.545 \\
& Uncoated & 0.082 & 0.065 & 0.017 & 0.628 \\
\hline $\mathrm{b}$ & Coated & 0.091 & 0.085 & 0.006 & 0.636 \\
& Uncoated & 0.104 & 0.082 & 0.022 & 0.873 \\
\hline $\mathrm{c}$ & Coated & 0.071 & 0.063 & 0.008 & 0.502 \\
& Uncoated & 0.073 & 0.054 & 0.019 & 0.646 \\
\hline $\mathrm{d}$ & Coated & 0.083 & 0.077 & 0.006 & 0.613 \\
& Uncoated & 0.088 & 0.069 & 0.019 & 1.001 \\
\hline
\end{tabular}


The MTF of two types of paper; coated and uncoated paper 150 $\mathrm{gr} / \mathrm{m}^{2}$ of test target 1 (Section 4.4.1), is calculated by using the $\mathrm{MTF}_{2 \mathrm{p}}$ model proposed in Equation (6.4). Figure 7.5 shows the obtained MTFs for both coated and uncoated paper. According to the MTF curves in Figure 7.5, it is obvious that the coated paper has wider MTF than the uncoated paper, which corresponds to less lateral light scattering compared to the uncoated paper.

The dot perimeter is calculated for all four dot samples $(a, b, c$, and d). Table 7.1 shows that, although the dots printed on the uncoated paper have less physical dot area, the dot perimeter is larger for the uncoated paper due to having an inhomogeneous dot shape. Thus, there are two factors that cause the uncoated paper to have bigger optical dot gain. One is that the light scatters more in the uncoated paper. The other one is that the ratio of the dot perimeter to its area is higher for uncoated paper.

Here, it can be concluded that the reason why the uncoated paper has more total dot gain compared to the coated paper in offset printing, is due to the optical dot gain and not the physical dot gain.

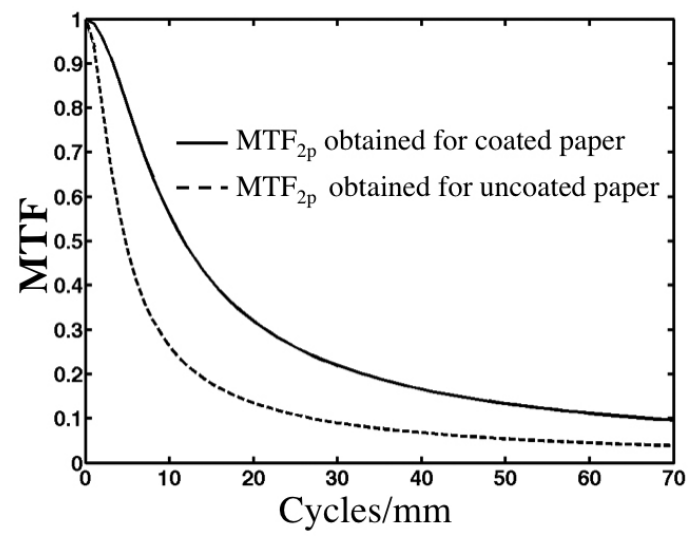

Figure 7.5: Comparison of $\mathrm{MTF}_{2 \mathrm{p}}$ for two types of paper; coated and uncoated paper.

\subsection{Dot Gain and Halftoning Techniques}

The investigation on the ink behavior for two types of paper in Section 7.3 has shown that although the ink spreads more on the coated paper, 
due to the light scattering effect and the dot perimeter, the dots printed on the uncoated paper has more total dot gain. Now, properties of three types of halftoning techniques with different dot sizes printed on coated and uncoated paper are characterized.

For this purpose five patches with $50 \%$ reference coverage are selected. These patches are halftoned by AM, FM2nd, and FM halftoning techniques. For the FM2nd technique, three different dot sizes are chosen and named regarding to the dot size which they produce; FM2nd_Big, FM2nd_Medium, FM2nd_Small. These patches are selected from test target 1, presented in Section 4.4.1 and printed on both coated and uncoated paper. Equation (5.11) is used for separating and illustrating the physical dot area of all patches. Figure 7.6 shows the physical dot area of the patches with $50 \%$ reference coverage. One should notice the fact that these patches may appear darker in the printed version of the dissertation. This is due to an additional dot gain caused by the print.

Table 7.2 verifies that for all types of halftoning the physical dot gain of patches printed on the coated paper is bigger than the uncoated. Table 7.2 also illustrates that the dots which are printed on the uncoated paper have a bigger perimeter compared to the coated paper. Due to the dots perimeter and the light scattering effect shown in Figure 7.5, the optical dot gain for the uncoated paper is bigger than the coated one. The above reasoning justifies the larger total dot gain for all types of halftoning techniques printed on the uncoated paper compared to the coated one.

Comparison of the physical dot gain of FM2nd technique with three different dot sizes show that by reducing the size of the dots, the ink area will be increased. Therefore, the total dots' perimeter is larger for the FM2nd with smaller dot size, resulting in a larger total dot gain.

In many of the halftoning techniques, it has been assumed that the halftone dots are circular, elliptical or square, but one should consider that the dots can be set to any shape. However, the shape of the dots might be changed, when two or more dots meet each other, hence there is a jump in dot size as the various dots merge. Due to the liquid state of the ink, when the size of the dots become bigger then there is a point where the ink drops eventually coalesce. Therefore in halftone prints this effect generally occurs more in the middle tones around $50 \%$ coverage. 


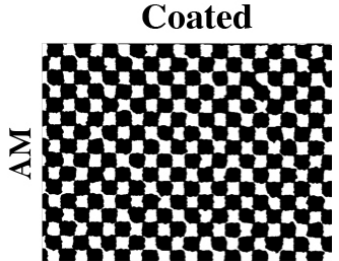

(a)

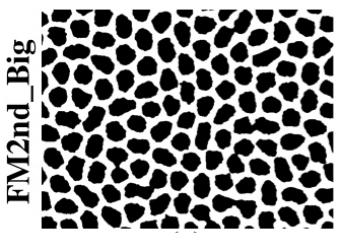

(c)

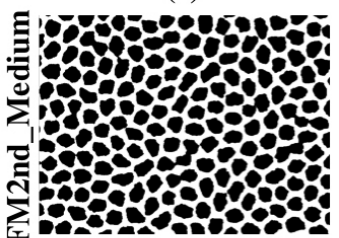

(e)

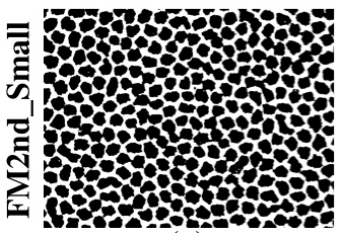

(g)

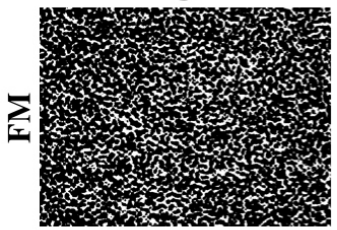

(i)

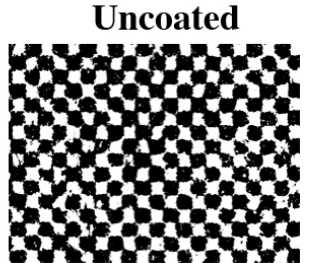

(b)

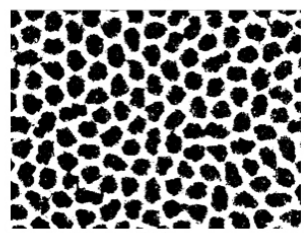

(d)

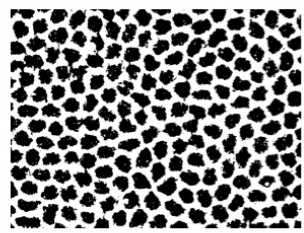

(f)

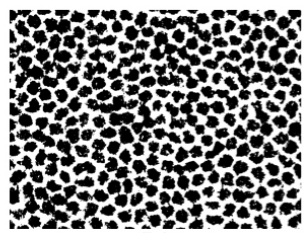

(h)

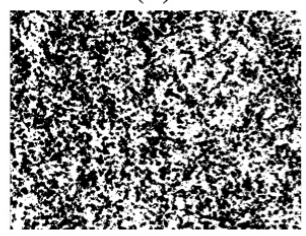

(j)

Figure 7.6: The physical dot area of $50 \%$ reference coverage patches halftoned by AM, FM2nd_Big, FM2nd_Medium, FM2nd_Small, and FM, printed on coated and uncoated paper.

Moreover the ink viscosity and cylinder pressure (in offset printing) [119], and shape of the dots are some important factors, which lead to overlapping dots when the distance between dots is reduced. In this case, the physical dot gain for the middle tones is bigger than the case where the dots are isolated from each other. 
Table 7.2: Comparison of the dot gains of AM, FM2nd_Big, FM2nd_Medium, FM2nd_Small, and FM halftoning techniques printed on coated and uncoated paper.

\begin{tabular}{|c|c|cc|c|}
\hline $\begin{array}{c}\text { Halftoning } \\
\text { Method }\end{array}$ & Paper Type & \multicolumn{2}{|c|}{ Dot Gain } & Perimeter \\
Total & Physical & $(\mathrm{mm})$ \\
\hline AM & Coated & 0.20 & 0.114 & 78.62 \\
& Uncoated & 0.23 & 0.074 & 118.87 \\
\hline FM2nd_Big & Coated & 0.14 & 0.056 & 71.24 \\
& Uncoated & 0.18 & 0.023 & 104.88 \\
\hline FM2nd_Medium & Coated & 0.16 & 0.058 & 86.29 \\
& Uncoated & 0.19 & 0.024 & 124.77 \\
\hline FM2nd_Small & Coated & 0.22 & 0.107 & 111.62 \\
& Uncoated & 0.24 & 0.035 & 154.97 \\
\hline FM & Coated & 0.36 & 0.113 & 196.11 \\
& Uncoated & 0.41 & 0.055 & 278.51 \\
\hline
\end{tabular}

To avoid the jump in the dot size at the middle tones, it is helpful to use another shape of the dot. The dots in the proposed FM2nd halftoning technique have different shapes and sizes. Figure 7.7 shows the original and printed dots halftoned by AM and FM2nd_Big halftoning technique. At $50 \%$ reference coverage, the dots halftoned by AM overlap with their adjacent dots, while in FM2nd_Big halftoning technique some of the dots are still isolated from each other.

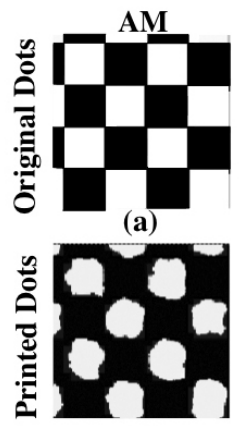

(c)

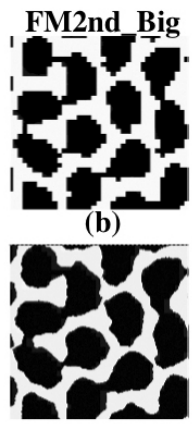

(d)

Figure 7.7: The original and printed dots (50\% reference coverage), halftoned by AM and FM2nd_Big methods.

The physical dot gain has been estimated for both AM and FM2nd_Big halftoning techniques. Figure 7.8 illustrates the physical dot gain of AM 
(150 lpi and $1200 \mathrm{dpi}$ ) and FM2nd_Big printed on the coated paper. As it can be seen in Figure 7.8, in AM halftoning, due to the overlapping dots, the physical dot gain has a peak at $50 \%$ reference coverage. On the other hand, in FM2nd_Big the dots are separated from each other and few dots have the chance to overlap at $50 \%$ reference coverage, due to their shapes. Therefore, the peak of the physical dot gain has been shifted to right compared to the peak for the AM halftoning technique.

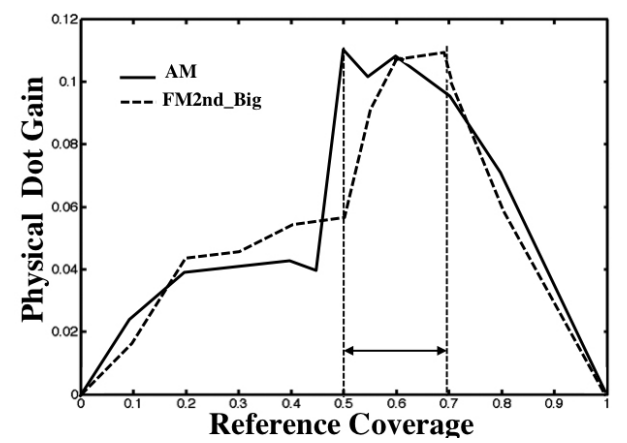

Figure 7.8: Physical dot gain of AM and FM2nd_Big on coated paper.

Figure 7.9 shows the physical and total dot gain of FM2nd halftoning technique with three different dot sizes on coated paper. As it can be seen in Figure 7.9 (a) and (b), the physical and total dot gains are not symmetric around $50 \%$ and their peaks are shifted to the right for all three different dot sizes; FM2nd_Big, FM2nd_Medium, FM2nd_Small.

It can be noticed in Figure 7.9 (a) that for the light gray tones, the physical dot gain is small, while for the dark gray tones the physical dot gain is relatively bigger. The reason is that in the dark gray tones, dots have more probability to overlap with their adjacent dots, whereas in the light tones the dots are separated from each other [119].

As it can be seen in Figure 7.9, the peak of the total dot gain is shifted around 10\% to the left compared to the peak of the physical dot gain. This is due to the symmetrical form of the optical dot gain versus the physical effective area [90]. Figure 7.9 (c) shows the optical dot gain versus physical effective area for FM2nd_Big, FM2nd_Medium, FM2nd_Small on coated paper. As can be seen the behavior of optical dot gain is symmetrical around $50 \%$ effective physical dot area. 


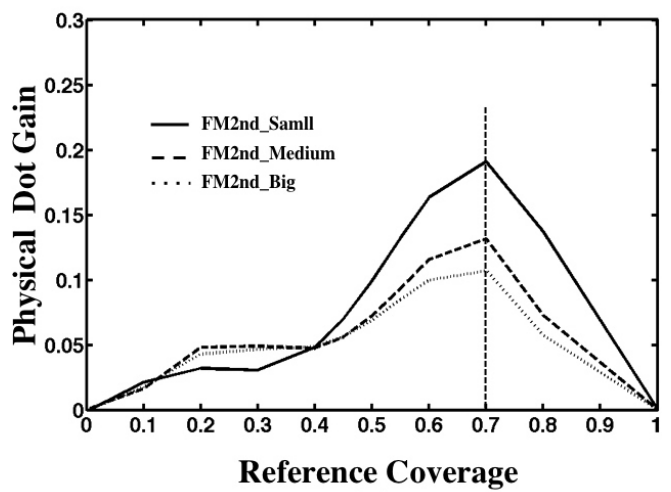

(a)

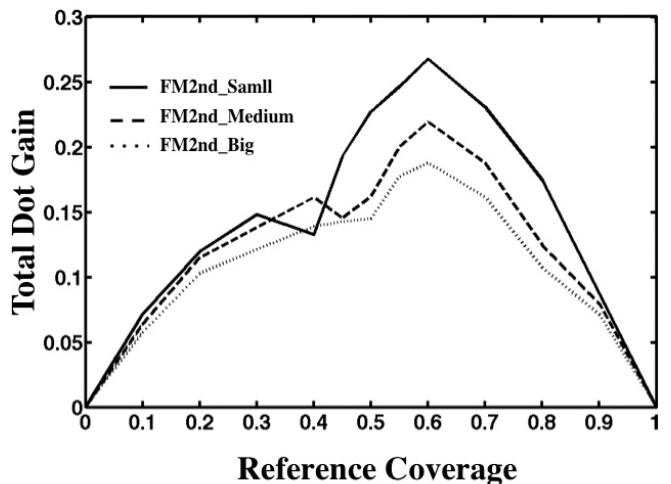

(b)

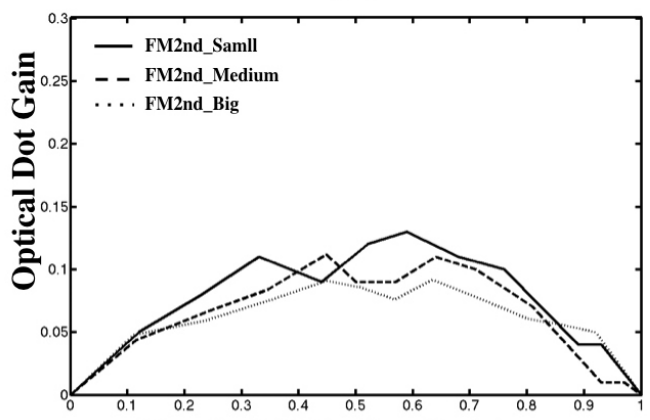

Physical Effective Dot Area

(c)

Figure 7.9: Comparison of (a) the physical and (b) the total dot gains for FM2nd_Big, FM2nd_Medium, FM2nd_Small on coated paper. (c) Optical dot gain versus physical effective dot area. 


\subsection{Color Gamut Determination}

Color gamut is an important characteristic for analyzing the range of reproducible colors for a device. The range of colors can be illustrated as a volume in the three-dimension color space. The gamut is usually specified in a colorimetric or visually based space such as CIEXYZ or CIELAB [9]. There exist several factors which affect the color gamut shape and size. In the case of printers, the printing technology, the characteristics of the colorants, the paper substrate, and the halftoning method are some of the examples of these factors [9]. In this section, the color gamut of different halftoning techniques printed with offset print press on coated and uncoated papers are computed and illustrated for comparison. However, it should be noticed that the study of color gamut is an extensive line of research, which is outside the scope of this dissertation.

To determine the color gamut of a printer, it is required to measure or predict the color of a number of samples. To get more accurate estimation, it is necessary to measure a large number of samples. In this study, by using the color prediction model presented in [35], the $L^{*} a^{*} b^{*}$ value of a large group of colors have been predicted. The $L^{*} a^{*} b^{*}$ value for all primary colors, cyan, magenta, and yellow is calculated for the reference coverages $0 \%, 5 \%, 10 \%, \ldots, 95 \%$, and $100 \%$ and all their possible combinations resulting in a total of 9261 different cases. The color prediction is carried out for all types of halftoning with different sizes presented in this dissertation; AM, FM2nd_Big, FM2nd_Medium, FM2nd_Small, and FM, which are printed on both coated and uncoated paper. The predicted colors are used to plot the three-dimensional visualization of the color gamut. The visualization of color gamut is implemented by using Matlab interface. The 3D Delaunay tessellation function is applied over all the CIELAB points. Figure 7.10 illustrates the 3D gridded boundary of the color gamuts for all proposed halftoning techniques, printed on both coated and uncoated paper. 
Coated

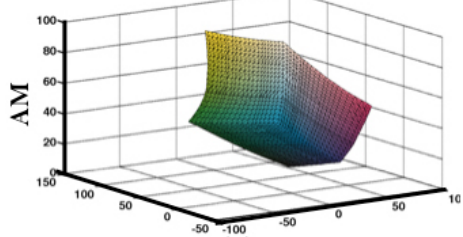

(a)

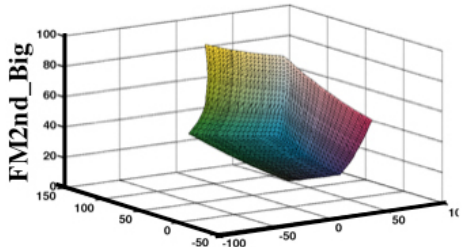

(c)

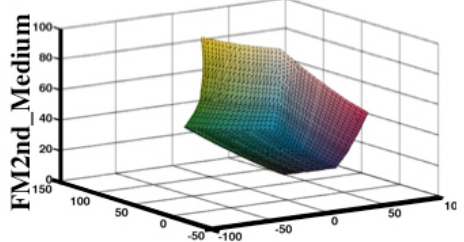

(e)

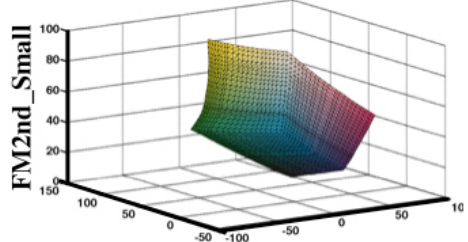

(g)

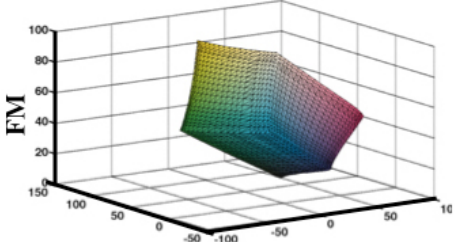

(i)
Uncoated

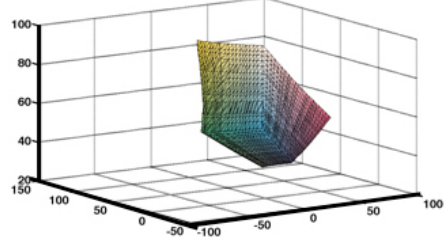

(b)

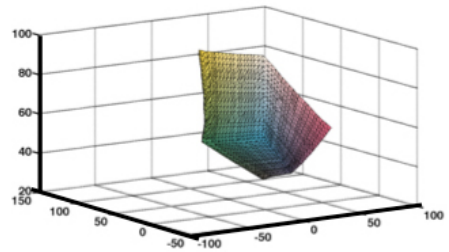

(d)

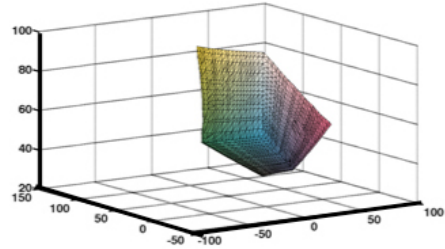

(f)

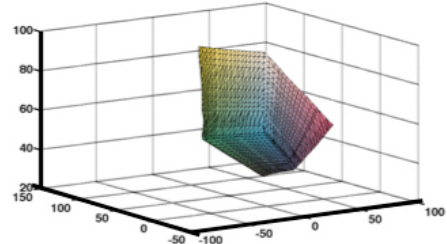

(h)

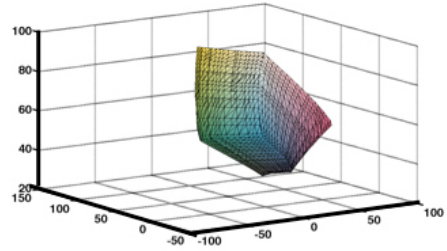

(j)

Figure 7.10: The color gamut of $\operatorname{AM}(\mathrm{a}, \mathrm{b})$, FM2nd_Big (c, d), FM2nd_Medium (e, f), FM2nd_Small (g, h), and FM (i, j), printed on both coated and uncoated paper, in CIELAB space. 


\subsubsection{Gamut Volume}

For color gamut numerical analysis, one needs to calculate the volume of the color gamut. One direct method for calculating the gamut volume, that has also been used in this study, is to set an interior point in the volume and sum up the volumes of the tetrahedrons. The tetrahedrons are created by connecting three points of the surface triangles to the interior point vertex. Figure 7.11 shows the scheme of tetrahedrons creation using an interior point.

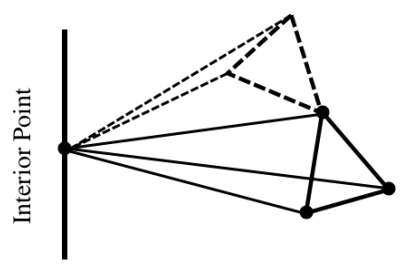

Figure 7.11: The scheme for tetrahedrons creation using an interior point.

Table 7.3 shows the estimated volume of the color gamuts shown in Figure 7.10. As it can be seen in Table 7.3 the volume of color gamut is increased from AM to FM2nd and then to FM1st. In the FM2nd halftoning technique, by increasing the dot size, the volume of the color gamut is decreased. Due to the greater ink absorption, the uncoated paper gives a lower ink density, which leads to lower color gamut volume, approximately half of the one obtained for the coated paper. It has been previously shown that a large dot gain can actually increase the color gamut [38]. Comparison between Table 7.2 and Table 7.3 also confirms the fact that by increasing the dot gain for the same paper the color gamut is also increased.

\subsection{Summary and Discussion}

In this chapter, the physical dot area was estimated for different dot shapes, produced by different types of halftoning techniques. The optical dot gain was simulated for all dot shapes by using the $\mathrm{MTF}_{2 \mathrm{p}}$ presented in Chapter 6. The results show the dependency of optical dot gain on the shape of the dots. By increasing the perimeter of the dot shapes, the optical dot gain is increased, however there is a limit to the ratio 
Table 7.3: The volume of gamut for AM, FM2nd_Big, FM2nd_Medium, FM2nd_Small, and FM, printed on both coated and uncoated paper.

\begin{tabular}{|l|lr|}
\hline Halftoning & \multicolumn{2}{|c|}{ Gamut Volume } \\
Technique & Coated paper & Uncoated paper \\
\hline AM & $3.2991 \times 10^{5}$ & $1.4522 \times 10^{5}$ \\
\hline FM2nd_Big & $3.3047 \times 10^{5}$ & $1.4251 \times 10^{5}$ \\
\hline FM2nd_Medium & $3.3487 \times 10^{5}$ & $1.4761 \times 10^{5}$ \\
\hline FM2nd_Small & $3.4236 \times 10^{5}$ & $1.5142 \times 10^{5}$ \\
\hline FM & $3.6136 \times 10^{5}$ & $1.6507 \times 10^{5}$ \\
\hline
\end{tabular}

of perimeter to area at which the optical dot gain will be saturated. Separately monitoring physical and optical dot gain can help the paper and graphic art industries to characterize the ink spreading on the paper and the optical dot gain effects.

In this chapter, the ink behavior and light scattering effect have also been investigated for the microscale dots produced by different halftoning techniques printed on coated and uncoated paper. The three dimensional demonstration of ink, has shown that the ink spreads more on the surface of the coated paper compared to the uncoated paper. The results show that the optical dot gain is not only dependent on the optical properties of the paper but also on the geometrical distribution of ink dots on the paper surface.

The color gamut has been illustrated for different halftoning techniques printed on two types of paper. Comparison of the color gamut volumes shows that the color gamut of the print on coated paper is bigger than the uncoated one. 


\section{Chapter 8}

\section{Characterization of Color Halftone Print}

Contents

8.1 Introduction $\ldots \ldots \ldots \ldots \ldots \ldots \ldots$

8.2 Primary and Secondary Ink Spectra . . . 123

8.3 Separation of Inks in Color Print . . . . . . 125

8.3.1 Omitting the Shadow Effect . . . . . . . . . . 129

8.4 Wavelength Dependency of Dot Gain in Color

Print . . . . . . . . . . . . 131

8.4.1 Wavelength Dependency of Light Scattering 135

8.5 Register Shift in Color Prints . . . . . 138

8.5.1 Register Shift Measurements . . . . . . . 140

8.6 Summary and Discussion . . . . . . . 142 


\subsection{Introduction}

The characteristics of primary inks (CMY) are far from ideal, and therefore characterizing the actual properties of color inks can be useful for system calibration and quality control in color reproduction. Many of the color prediction models consider that the characteristics of a color ink printed on paper differs from the characteristics of the same ink printed together with another ink $[24,47,97]$. By separating two color inks of a color print from each other, it is possible to study some ink characteristics such as ink spread and penetration of each individual ink. In the reflective wavelength band of a color ink, the ink transmits the incoming light, and thereby becomes barely visible. Moreover, in the absorbing wavelength band of a color ink, the ink absorbs the light, and hence becomes completely visible. This fact is used to separate two printed color inks from each other. Since the inks are not ideal, it is sometimes impossible to find a wavelength band at which one color ink completely absorbs the light and the other one completely transmits it. Therefore, the effect of one ink is not completely removed when sending the light at the absorbing wavelength band of the other ink. In this case, a shadow of the ink remains in the microscale image of the two inks. In spectral measurements using a spectrophotometer, the term shadow effect of one ink means the contribution of that ink to the reflectance spectra of the other ink. For example for the blue patch (cyan and magenta), at the absorbing wavelength band of magenta the spectral reflectance of cyan also has some contribution, which is called the shadow effect of cyan in microscale images. Due to this fact, using a spectrophotometer to measure the reflectance of the color patches in different wavelength bands is not a proper approach to study the characteristic of each color ink when printed together with others. In this chapter, first a method is presented to separate color inks from each other, then a new approach is presented to measure the register shift in color printing. The study in this chapter has been previously published in $[36,76,77]$.

\subsection{Primary and Secondary Ink Spectra}

The color ink, which is printed on a substrate, partially reflects the light in some wavelengths and absorbs the rest. This means that the color ink acts as a filter in the visible wavelength band (380 nm-780 $\mathrm{nm})$. In this section, 21 halftone patches of each primary (CMY) and 
secondary (RGB) inks of test target 2 (Section 4.4.2) are selected. The used reference coverages of each ink are; $0 \%, 5 \%, 10 \%, \ldots, 95 \%, 100 \%$.

Figure 8.1 shows the absorbing and reflective wavelength band of primary and secondary color inks. It should be noted that the black ink (not shown in Figure 8.1) absorbs all the visible wavelengths but cyan, magenta, and yellow only absorb some part of the visible wavelengths.

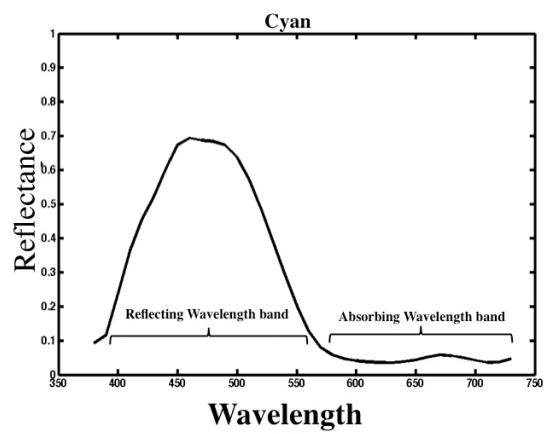

(a)

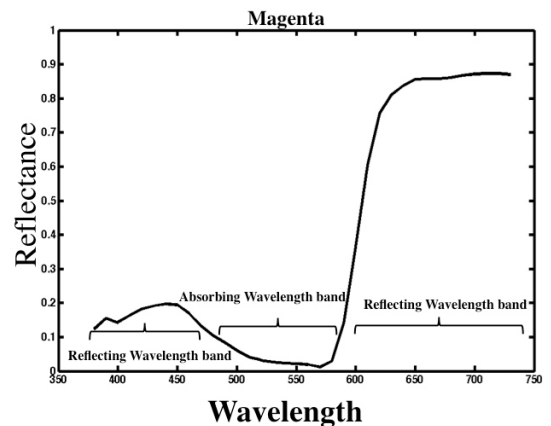

(b)

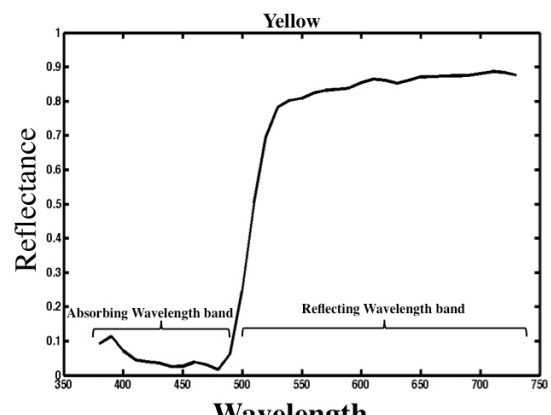

Wavelength

(c)

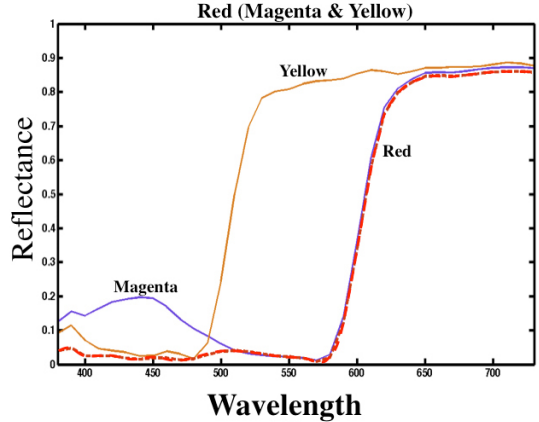

(d)

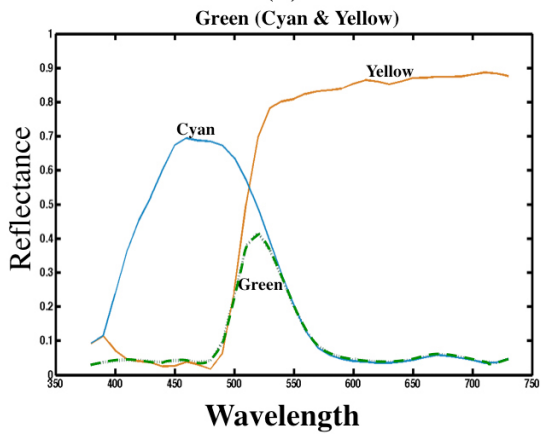

(e)

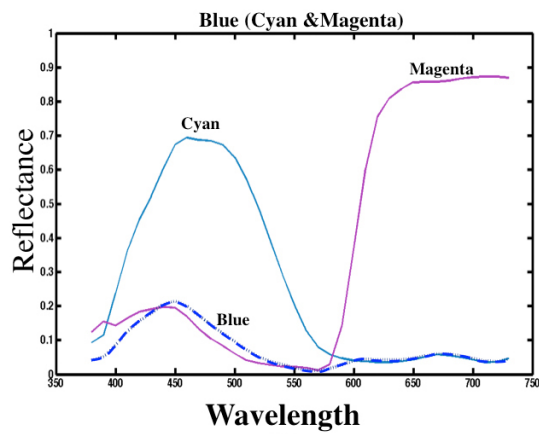

(f)

Figure 8.1: Absorbing and reflective wavelength bands of color inks. (a) Cyan. (b) Magenta. (c) Yellow. (d) Red. (e) Green. (f) Blue. 


\subsection{Separation of Inks in Color Print}

For analyzing the color inks' behavior, the microscale multi-channel images are captured in different wavelengths. In Chapter 4 the multichannel image acquisition system was presented in details. For two-color patches, by illuminating the light in the reflective wavelength band of one of the two inks, it is possible to neglect the effect of that ink and have only the second one. Figure 8.2 shows the microscale image of green dots, i.e. cyan dots printed on the yellow dots, and their reflectance spectra. By illuminating the light in the reflective wavelength band of cyan, for example $450 \mathrm{~nm}$, the cyan dots will vanish and only the yellow dots will remain. By moving towards the reflective wavelength band of yellow it can be seen that the yellow dots become pale and at $650 \mathrm{~nm}$ the yellow dots completely vanish and cyan dots appear. Note that at $700 \mathrm{~nm}$ the yellow dots will also disappear.

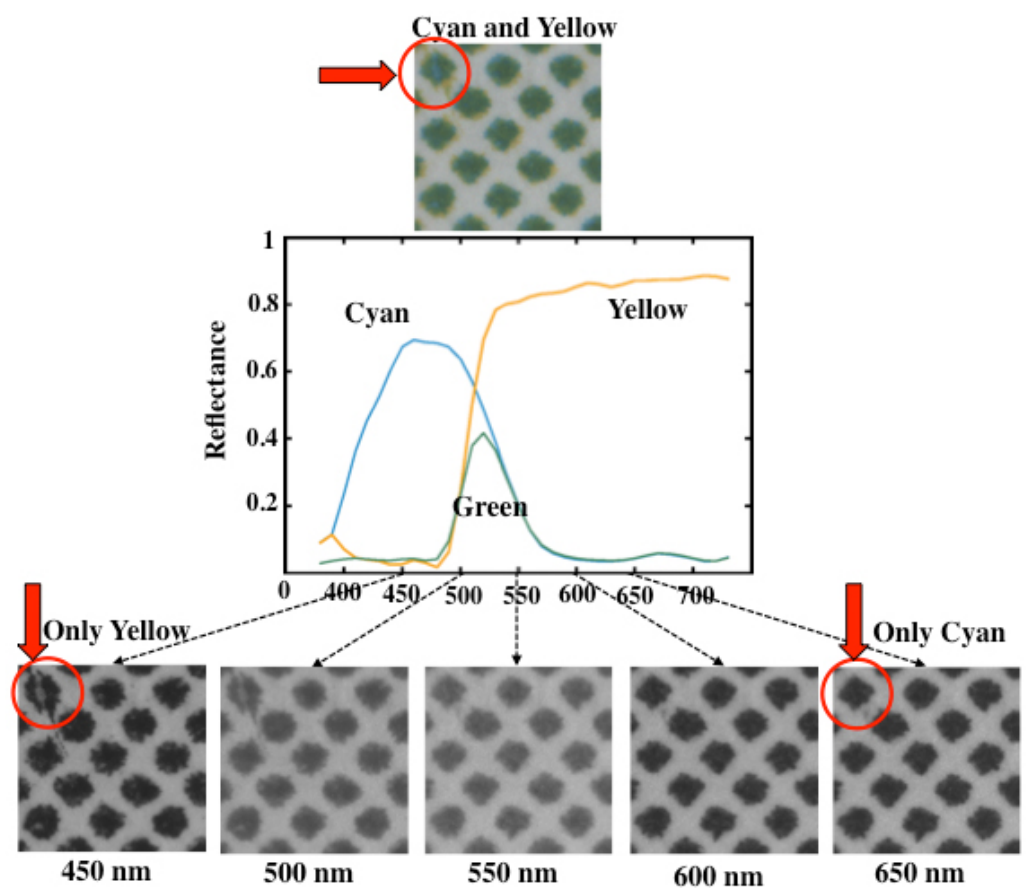

Figure 8.2: The microscale image of green patch. By moving from the reflective wavelength band of cyan to the reflective wavelength band of yellow, it can be seen that the yellow dots become pale and at $650 \mathrm{~nm}$ the yellow dots completely vanish and cyan dots appear. 
Figure 8.3 shows the yellow and cyan dots (30\% reference coverage) which are printed dot-off-dot as much as possible on a coated paper. The total dot gain of cyan at $700 \mathrm{~nm}$, when printed with yellow is compared with the total dot gain of only cyan patches at the same wavelength. Figure 8.3 (c) shows the dot gain curve of the green patches and the cyan patches obtained by the approach presented in Section 5.3.2 (with full tone stripe) at $700 \mathrm{~nm}$. This comparison validates the fact that, by illuminating the light in the reflective wavelength band of one of the two color inks, it would be possible to separate them from each other. In this case when two color inks are printed dot-off-dot on coated paper the dot gain of inks is close to the dot gain of the same ink when it is printed separately on the coated paper. However, the dot gain might be different when the dots are printed on top of each other (dot-on-dot) or when they are printed on an uncoated paper, in which the penetration becomes an important factor that might affect the dot gain characteristics.

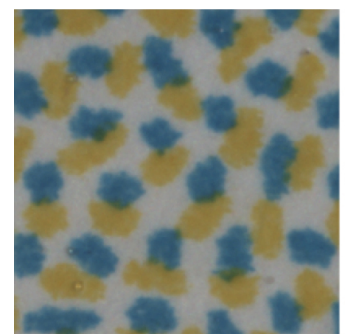

(a)

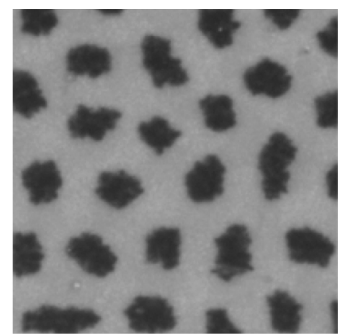

(b)

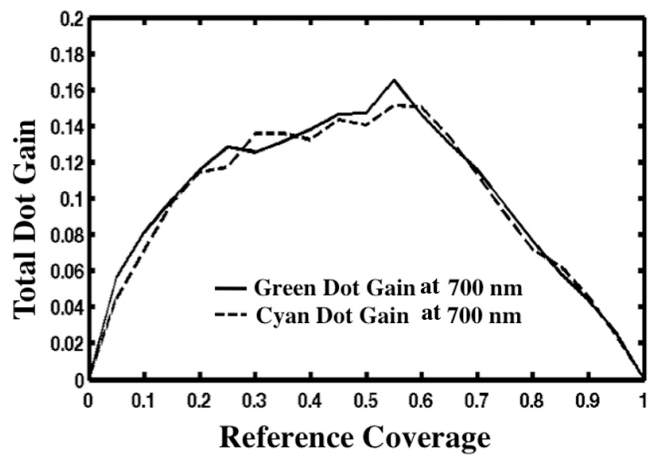

(c)

Figure 8.3: (a) Cyan and yellow dots (dot-off-dot) printed on a coated paper. (b) The same patch is captured at $700 \mathrm{~nm}$. (c) Dot gain of green patch and only cyan ink at $700 \mathrm{~nm}$ 
Since the color inks are not ideal, and the availability of filters in our image acquisition system for different wavelengths is limited, sometimes it becomes hard to find an exact wavelength to separate the two color inks. For example, by looking at the reflectance spectra in Figure 8.4 (the blue patch), it can be observed that at $700 \mathrm{~nm}$, magenta will disappear but at either $500 \mathrm{~nm}$ or $550 \mathrm{~nm}$ (the absorbing wavelengths of magenta), it is not possible to fully separate cyan from magenta. The reason is that at $500 \mathrm{~nm}$, although cyan is somewhat transparent, the magenta ink does not completely absorb the light, and at $550 \mathrm{~nm}$, the cyan ink also absorbs most of the incoming light, see Figure 8.4. The contrast between cyan and magenta dots is more evident at $500 \mathrm{~nm}$, therefore this wavelength band is used to capture magenta dots.

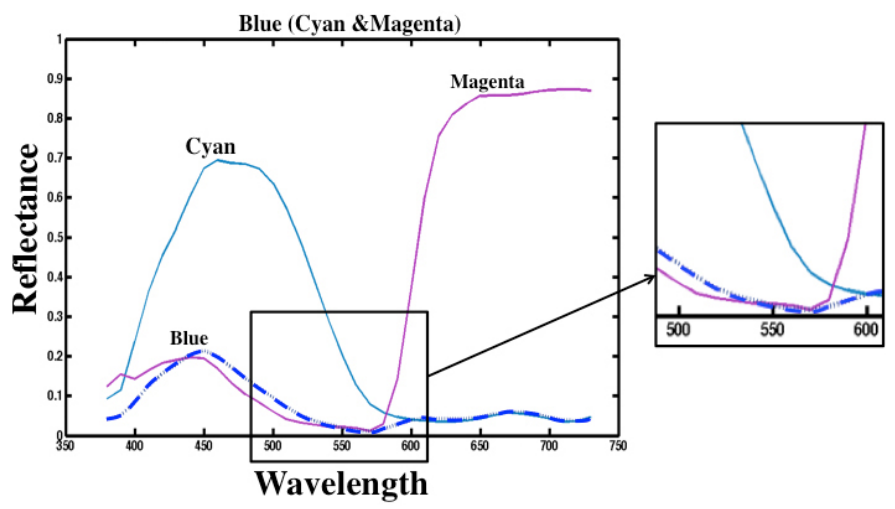

Figure 8.4: Reflectance spectra of cyan and magenta.

Figure 8.5 (a) shows the microscale image of a blue patch; cyan and magenta with $20 \%$ reference coverage, which were designed according to dot-on-dot strategy in the bitmap. It should be noted that due to the misregistration, the cyan and magenta dots in Figure 8.5 (a) are shifted in position. Figure 8.5 (b) illustrates the same image using the filter at $700 \mathrm{~nm}$. Here, one can only see the cyan dots, while the magenta dots are not visible. Figure 8.5 (c) shows the captured image at 500 $\mathrm{nm}$, and in this figure the magenta dots are clearly visible together with the shadow of cyan dots. This shows that at the absorbing wavelength band of cyan, magenta has no effect, but at the absorbing wavelength band of magenta, cyan has an impact. In other words, due to the effect of cyan's shadow, the dot gain for magenta (in blue patch) at $500 \mathrm{~nm}$ is bigger than the dot gain of only magenta dots at $500 \mathrm{~nm}$. 


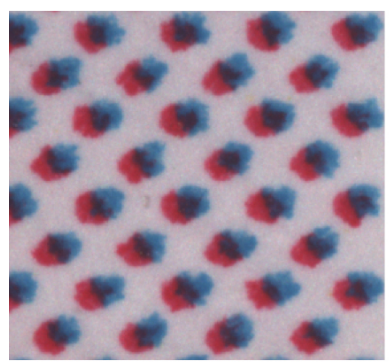

(a)

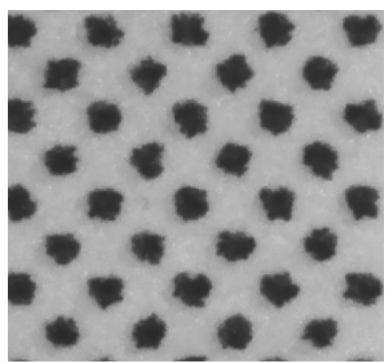

(b)

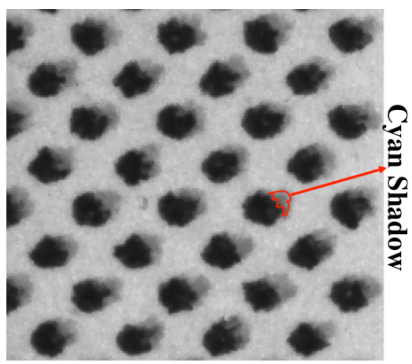

(c)

Figure 8.5: (a) Microscale image of blue patch (dot-on-dot in the bitmap). (b) Blue at $700 \mathrm{~nm}$ (only cyan dots). (c) Blue at $500 \mathrm{~nm}$ (magenta dots with the shadow of cyan dots).

As it can be seen in Figure 8.5 (c) the effect of cyan's shadow also has an impact on the average of reflectance value of the halftone patch. Therefore the total dot gain of blue ink at $500 \mathrm{~nm}$ includes both dot gain of magenta and the effect of cyan's shadow, while at $700 \mathrm{~nm}$, only the cyan dots affect the total dot gain. It is obvious that the total dot gain of blue patch at $500 \mathrm{~nm}$ would become bigger than the total dot gain of blue at $700 \mathrm{~nm}$, see Figure 8.6.

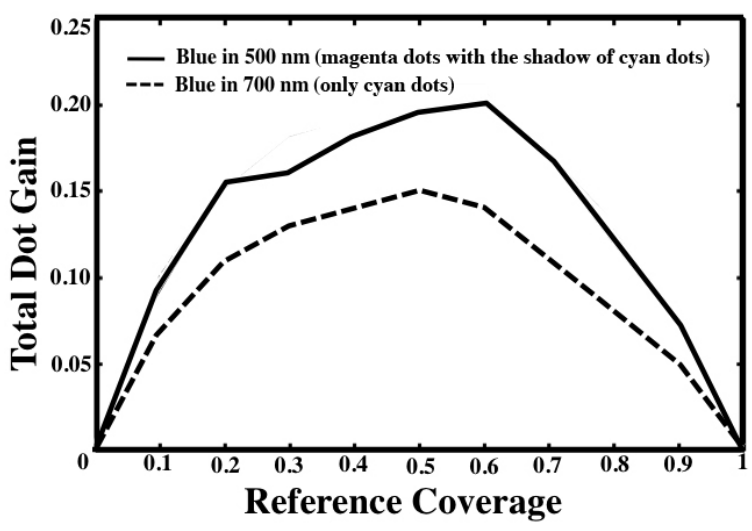

Figure 8.6: Solid line, total dot gain of blue patch at $500 \mathrm{~nm}$ (magenta dots with the shadow of cyan dots). Dashed line, total dot gain of blue patch at $700 \mathrm{~nm}$ (only cyan dots).

According to the above discussion, the two inks' reflectance spectra measured by spectrophotometer will also be affected by the "shadow 
effect". In order to compare the shadow effect in the microscale images and contribution of reflectance spectra in the spectrophotometer results, the total dot gain of blue patches has been computed by using both reflectance spectra and microscale images. Figure 8.7 shows the total dot gain of blue patches obtained by illuminating the light at $500 \mathrm{~nm}$ (magenta). The reflectance values of $R_{a v e}, R_{p}$, and $R_{i}$ are measured by both spectrophotometer and high-resolution camera. The total dot gain is obtained by using Murray-Davies equation for the blue patches at 500 $\mathrm{nm}$. As seen in Figure 8.7 the results are close to each other. This shows that, there is a contribution from cyan to the reflectance spectra in the spectral measurements, measured by spectrophotometer. Now it can be concluded that using the spectrophotometer to measure color patches can not be an appropriate approach to separate the effect of cyan on magenta at some wavelengths.

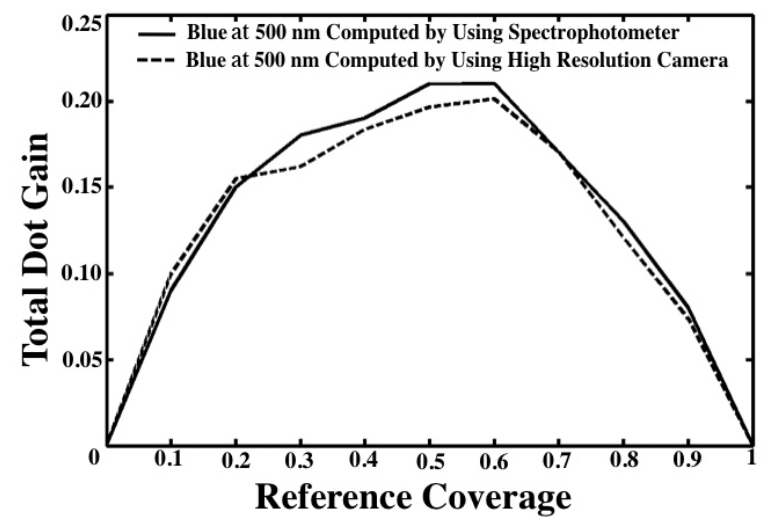

Figure 8.7: Total dot gain of magenta (blue at $500 \mathrm{~nm}$ ). Solid line: total dot gain obtained by using spectrophotometer. Dashed line: total dot gain obtained by using high-resolution camera.

\subsubsection{Omitting the Shadow Effect}

In this section, the MIH approach presented in Chapter 5, is used to remove the shadow effect. Figure 8.8 (a) and (b) show a blue patch; cyan and magenta with $20 \%$ reference coverage, at $500 \mathrm{~nm}$ and its reflectance histogram. There are three peaks in the histogram; the first peak corresponds to the magenta dots, the second one to the shadow of cyan, and the third one to the paper between dots. 


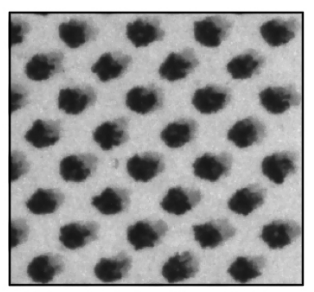

Magenta dots + Cyan shadow

(a)

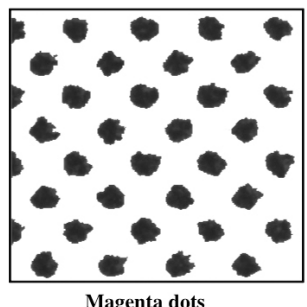

(c)

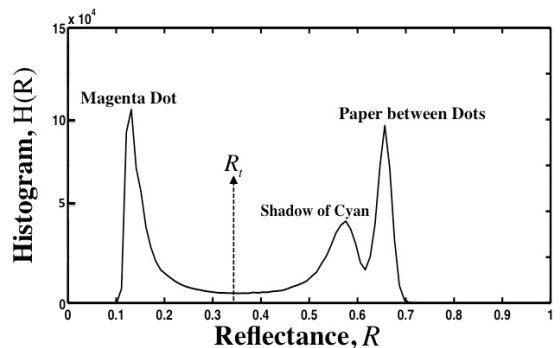

(b)

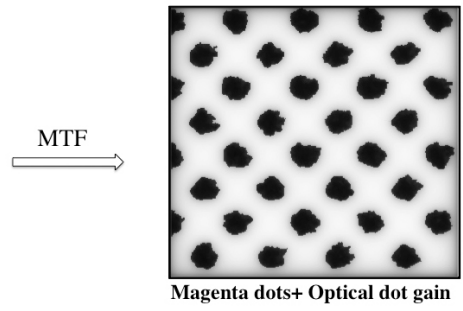

(d)

Figure 8.8: (a) Blue patch at $500 \mathrm{~nm}$ (magenta dots with the shadow of cyan dots). (b) Reflectance histogram of blue patch at $500 \mathrm{~nm}$. (c) Magenta dots after removing the cyans shadow. (d) Simulated optical dot gain using MTF.

The MIH approach is used to find the position, which has the minimum value on the reflectance histogram between the peaks of magenta and the shadow of cyan. The minimum point, $R_{t}$, in the histogram indicates the boundary between the magenta dots and the rest of the surface (i.e. paper, optical dot gain and the shadow of cyan). By using Equation (8.1), it is possible to graphically illustrate the physical dot area of magenta, on which the cyan's shadow has no impact.

$$
\operatorname{Ink}_{\text {magenta }}= \begin{cases}0 & \text { if } \quad I_{\text {blue }}(x, y)<R_{t} \\ 1 & \text { otherwise }\end{cases}
$$

In Equation (8.1), $I_{b l u e}(x, y)$ indicates the microscale image of the blue patch at $500 \mathrm{~nm}$, and the threshold $R_{t}$ is found by using the MIH approach. Figure 8.8 (c) shows the magenta dots, without any effect of

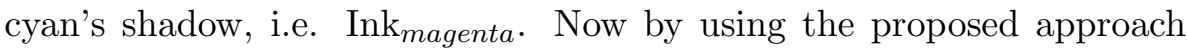
the two color inks have been separated from each other and the physical dot area of each color ink can be studied individually. 
By using Equations (7.1) and (7.2), and set $d_{2}=0.117$, which is computed for the coated paper $150 \mathrm{gr} / \mathrm{m}^{2}$, the optical dot gain is simulated for magenta dots, see Figure 8.8 (d). The total dot gain of the blue patches after omitting the shadow effect is thereby calculated. Figure 8.9 shows the total dot gains of blue patch before and after omitting the shadow effect. It is obvious that the total dot gain is reduced after the shadow effect removal.

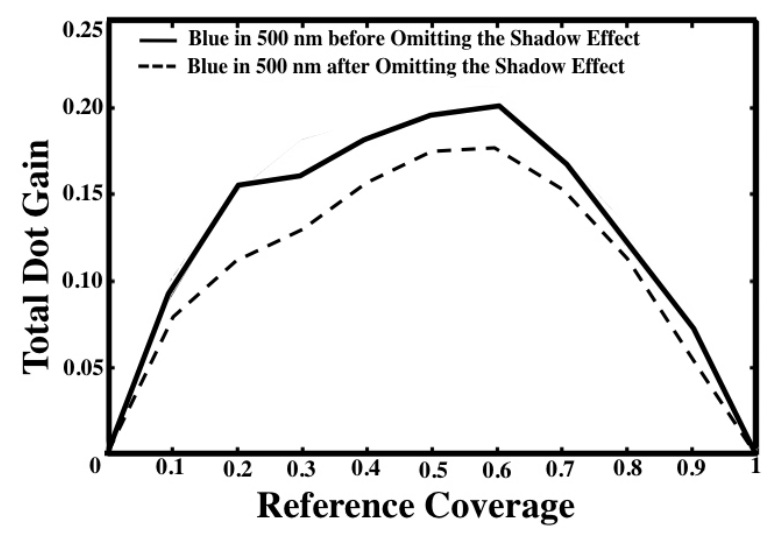

Figure 8.9: The total dot gain of blue patch at $500 \mathrm{~nm}$ before and after omitting the shadow effect.

\subsection{Wavelength Dependency of Dot Gain in Color Print}

The term of light scattering refers to all physical processes that move photons apart in different directions. This phenomenon is caused by variation of refractive index within a non-homogeneous medium. In 1871, Lord Rayleigh, established the Rayleigh-scattering theory which the amount of Rayleigh scattering depends on the size of the particles and the wavelength of the light $[11,103]$. The intensity, $I$, of the scattered light is given by,

$$
I=I_{0}\left(\frac{1+\cos ^{2} \theta}{2 R^{2}}\right)\left(\frac{2 \pi}{\lambda}\right)\left(\frac{n^{2}-1}{n^{2}+2}\right)^{2}\left(\frac{d}{2}\right)^{6}
$$


where $I_{0}$ is the light intensity before having an interaction with the particle, $\mathrm{R}$ is the distance between the particle and the observer, $\theta$ is the scattering angle, $n$ is the refractive index of the particle, and $d$ is the diameter of the particle. It can be seen from Equation (8.2) that Rayleigh scattering is dependent on the size of the particle and the wavelengths. The intensity of the Rayleigh scattered radiation increases when the ratio of particle size to wavelength increases. In the Rayleigh theory the scattering coefficient depends on the wavelength of light, which means that the blue light is scattered more strongly than the red light. The scattered sunlight in the atmosphere is mostly blue, which explains why the sky is blue. The Rayleigh scattering model is not applicable when the particle size becomes larger than around $10 \%$ of the wavelength of the incident light.

In 1908, Gustav Mie proposed a theory which generalizes the Rayleigh model [70]. The Mie-scattering theory, which is derived from Maxwells' equations, can be compared to Rayleigh scattering theory by emphasizing on this fact that the light scattering is wavelength independent for mediums with particles bigger than the light wavelength. According to the Mie theory, the light scattering in the paper which is made by particles bigger than the light wavelengths can roughly be wavelength independent. Previously, it has been shown that the photons with short wavelength can not penetrate in to deeper points of paper. Due to its strong power of scattering, it has been claimed that the MTF of paper is wider for shorter wavelengths, but the difference is not significant [110].

Since it is possible to separate the physical and optical dot gains with MIH approach, one can further study the dependency of optical dot gain for different wavelengths. For this purpose, the multi-channel images captured by the high-resolution camera have been used. In the multichannel image capturing, the light intensity, which is passed through the filters and is illuminated on the substrate, can be obtained as follows,

$$
I_{F_{n}}(\lambda)=I_{s}(\lambda) \cdot F_{n}(\lambda)
$$

where $I_{F_{n}}(\lambda)$ represents the transmitted light through the filter and $I_{s}(\lambda)$ indicates the intensity of the light source. The $(\lambda)$ variable indicates that $I_{F_{n}}(\lambda), I_{s}(\lambda)$, and $F_{n}(\lambda)$ are functions of wavelength. $F_{n}(\lambda)$ denotes the characteristic of the filter where the subscript $(n)$ represents the filter 
number. By using the Murray-Davies equation the total dot gain for each color ink at different wavelengths is calculated as follows,

$$
a_{e f f}=\frac{R_{F_{n}}(\lambda)-R_{p, F_{n}}(\lambda)}{R_{i, F_{n}}(\lambda)-R_{p, F_{n}}(\lambda)}
$$

where $R_{F_{n}}(\lambda), R_{p, F_{n}}(\lambda)$, and $R_{i, F_{n}}(\lambda)$ represent the average values of halftone patch, unprinted stripe, and $100 \%$ ink stripe, respectively.

Figure 8.10 shows the total dot gains of cyan, magenta, and black at different absorbing wavelengths. It is illustrated that the total dot gains are different at different absorbing wavelengths, for cyan and magenta. However for black ink the total dot gains are quite close to each other, by a maximum difference less than $0.4 \%$ which is not significant. It must be added that the black patches were also measured by spectrophotometer, and the total dot gains at different wavelengths were obtained. Even the spectral measurements confirm that the difference between the dot gains at different wavelengths is very small. Since the physical dot gain is surely wavelength independent, if the light scattering was highly wavelength dependent, there should have been more significant difference between the total dot gains of black patches at different wavelengths. This will be discussed in more detail in Section 8.4.1.

The total dot gain in Figure 8.10 (c) is computed by using six interference filters. The black ink absorbs all wavelengths in visible spectrum. The light source used in this experiment had a low intensity in the wavelength band of $350 \mathrm{~nm}-400 \mathrm{~nm}$, therefore the first filter, with center wavelength of $400 \mathrm{~nm}$ is neglected. The reflectance value of yellow dots which are printed on the paper are close to the reflectance value of paper compared to cyan and magenta. Therefore, according to Equation (8.4) the numerator and denominator of the fraction are both approaching zero, which makes it hard to compute the dot gain of yellow ink at the wavelength between (480 nm -700 nm). Therefore, in this section only the cyan, magenta, and black color inks are considered to be investigated for the wavelength dependency of their dot gains.

Since each color ink has its own absorbing wavelength band, the behavior of its dot gain might vary dependent on the wavelength [35]. For all color inks we have calculated dot gain in two steps: 1 . Without any filter 2 . With the seven interference filters. Figure 8.11 shows that among all filters, the filter corresponding to maximum dot gain at the absorbing wavelength band of a specific ink almost gives the same dot 


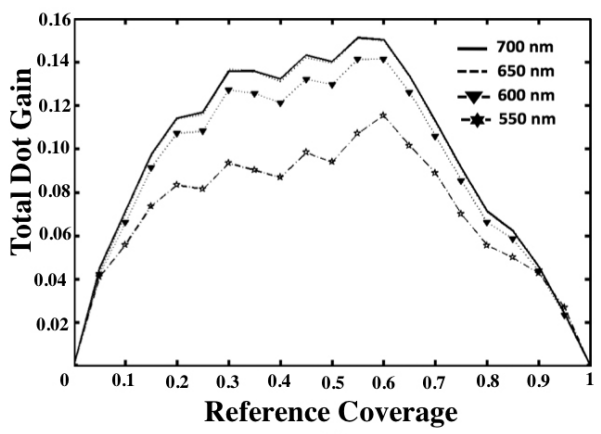

(a)

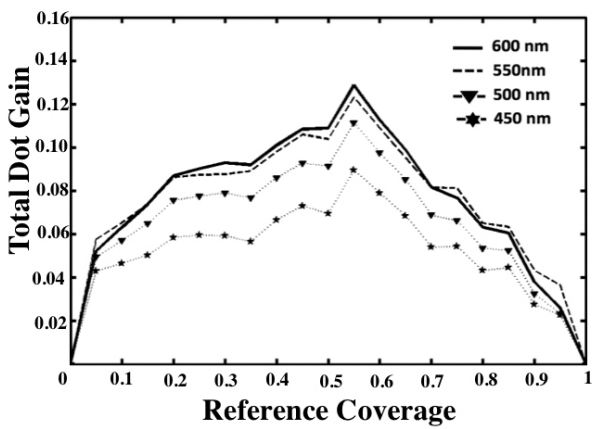

(b)

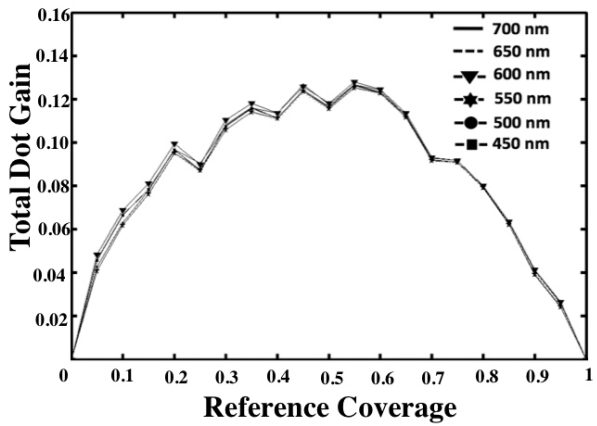

(c)

Figure 8.10: Total dot gains in the absorbing wavelength band. (a) Cyan ink. (b) Magenta ink. (c) Black ink.

gain value as when we are not using any filter. For cyan, magenta, and black the maximum difference between the dot gain at absorbing wavelength band and the dot gain using no filter are only $0.1 \%, 0.9 \%$, and $0.1 \%$, respectively. 


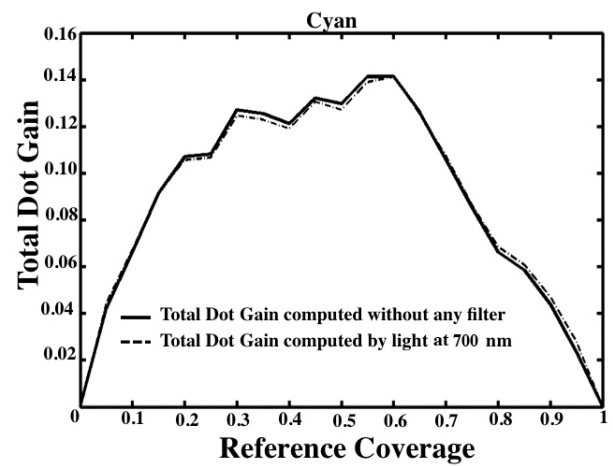

(a)

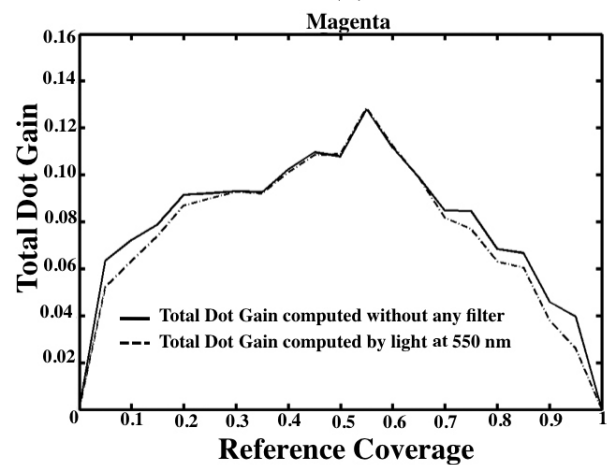

(b)

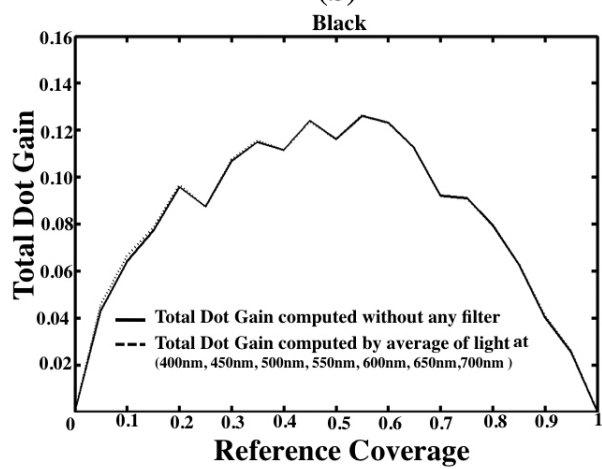

(c)

Figure 8.11: Dot gain is calculated in two steps: Solid line: without any filter, Dashed line: with filter. (a) Cyan, (b) Magenta, (c) Black

\subsubsection{Wavelength Dependency of Light Scattering}

As introduced in Section 3.3.1, the physical dot gain is a result of the real physical extension of ink dot. Therefore, the wavelength of the 
light has no effect on the physical dot gain. In this section, in order to study the wavelength dependency of light scattering, the physical dot gain is separated by using the MIH approach for black ink. Figure 8.12 illustrates the total and optical dot gains for black ink at different wavelengths, halftoned by AM halftoning technique printed on a coated paper. It can be observed that the optical dot gain for black at different absorbing wavelength bands are quite close to each other by a difference around $1 \%$. It can also be observed that the difference between the optical dot gain at lighter tones are bigger than that of darker tones. The reason is that the effective dot area $\left(a_{e f f}\right)$ for tiny dots are small and it is difficult to accurately separate the physical dot gain from the total one.

The optical dot gain curves for black ink are very close for all absorbing wavelength bands. Although the particles making different types of paper are variant in size, the light scattering effect, which is the reason for optical dot gain creation, must be less sensitive to different wavelength bands [76]. In 2010, Ukishima studied the dependency of MTF for the inkjet printing paper at different wavelength bands [108]. He showed that a shorter wavelength has a wider MTF, but the difference between the MTFs is not significant.

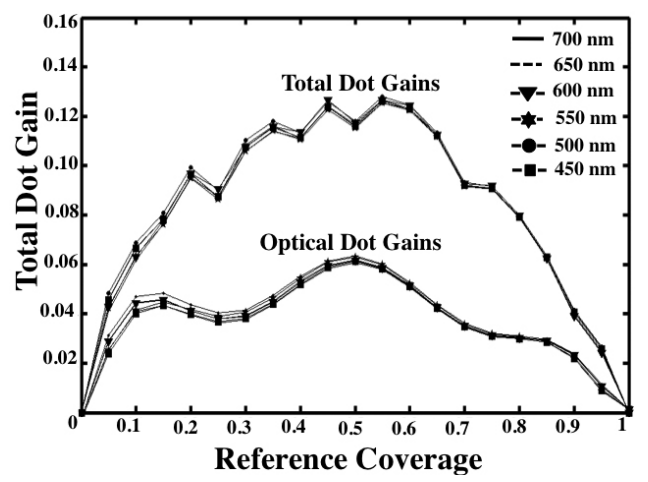

Figure 8.12: Total and optical dot gains for black ink at different wavelengths, halftoned by AM halftoning technique printed on a coated paper.

Now the question is, why the total dot gain differs for color inks (magenta and cyan) in different wavelength bands. The Murray-Davies model requires making a fundamental assumption that both the substrate and the ink are of uniform color. It is important to note that, 
this is rarely the case. For typical printing on plain paper, dot densities are not uniform [113]. For a print without ink penetration, the reflected light passes twice through the ink layer. As the ink itself scatters the light, there is a significant loss in the light, therefore the behavior of ink transmittance at different wavelengths plays an important role in the reflectance value. To study the ink behavior at different wavelengths, Equation (8.5) is used to express the logarithmic form of the transmittance,

$$
D(\lambda)=-\log _{10} T(\lambda)
$$

where $D(\lambda)$ is the spectral density corresponding to the transmittance $T(\lambda)$. In the density scale, $D(\lambda) \approx 0$ corresponds to the reflective wavelength band of the ink and the values of $D(\lambda)$ increase logarithmically in the absorbing wavelength band. An extreme case for the ideal ink is when the absorbing wavelength band corresponds to an infinite density, $D(\lambda) \approx \infty$, which happens when $T(\lambda)$ approaches zero. Figure 8.13 (a) and (b) show the density spectrum of full tone patches of cyan and magenta printed on the coated paper. As seen in Figure 8.13 (a) and (b) the ink density is not constant in the absorbing wavelength band. Therefore according to Equation (8.5), the ink transmittance is not constant in the absorbing wavelength band either. Therefore, due to the variation of ink transmittance values in the absorbing wavelength band, the reflected light from the printed ink differs for different wavelength band, which results in different total dot gain. Notice that if the color inks were ideal their total dot gain would be equal for all absorbing wavelengths as it is for black ink.

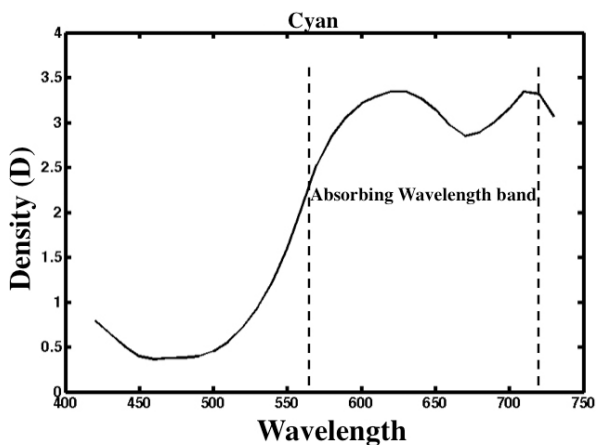

(a)

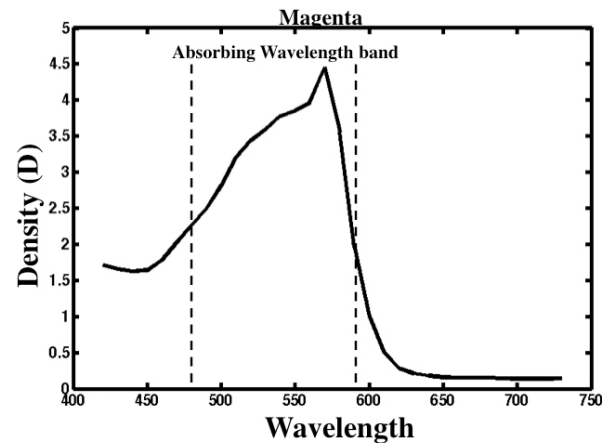

(b)

Figure 8.13: Ink density in different wavelengths. (a) Cyan.

(b) Magenta. 


\subsection{Register Shift in Color Prints}

Due to the imprecise transportation of the printing substrate in commercial prints the halftone dots are seldom placed exactly at their corresponding positions in the digital bitmap [41]. This will certainly cause some color shift in the final print result. In practice, it is impossible to predict the direction and the size of the register shift, which makes it impossible to compensate for the color shift caused by the register shift. In AM halftoning, in order to reduce the impact of register shift, instead of only using one halftone angle, four different angles are used for the process inks, cyan, magenta, yellow and black. The reason is that when the same angle is used, the color dots are printed on top of each other (dot-on-dot) and a shift in position for one of the inks can move the print towards dot-off-dot, which can cause a large color difference [34]. When the angles are different, the print is not dot-on-dot and a shift in position will possibly have smaller impact on the final color. The FM halftoning, when the separations are halftoned independently is hardly sensitive to register shift. The reason is the fact that dots are small and placed somewhat stochastically and the color channels are halftoned independently. Therefore any shift in positions will not have a great impact on the resulting color. When it comes to the second generation FM2nd halftoning, where not only the frequency (number) of the dots is variable but also their size varies, one could predict that the sensitivity to be somewhere between AM and FM halftoning.

In order to carry out a thorough investigation on the sensitivity of different halftoning methods to register shifts, the halftone patches of cyan and magenta using AM, FM and FM2nd are created. Figure 8.14 shows a $20 \%$ halftone patch being halftoned by AM (at $15^{\circ}$ ), FM and FM2nd halftoning methods and printed at $100 \mathrm{dpi}$ for illustration purposes. In the case of AM, two different angles for dot placement are used; the same angle of $45^{\circ}$ for both cyan and magenta and also $15^{\circ}$ for cyan and $75^{\circ}$ for magenta. In the case of FM and FM2nd the cyan and magenta channels were halftoned independently. Two different register shifts scenarios are simulated; two pixels and five pixels in both $\mathrm{X}$ and $\mathrm{Y}$ directions in the digital bitmap. These shifts correspond to 169 and 423 $\mu \mathrm{m}$ as the patches are printed at $300 \mathrm{dpi}$. The spectrophotometer is used to measure the CIELAB values for the printed halftoned patches with and without simulated register shift. By calculating the $\Delta E_{a b}$ color difference between the color of each patch before and after simulated 
register shift one can determine how sensitive different halftones are to register shift. Totally 33 different patches with different coverage for cyan and magenta were printed. Table 8.1 illustrates the $\Delta E_{a b}$ caused by register shift of 2 and 5 pixels in both $\mathrm{X}$ and $\mathrm{Y}$ directions for different halftoning methods.

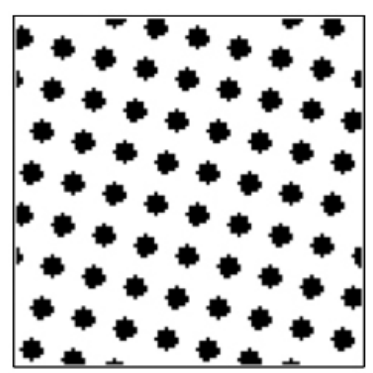

(a)

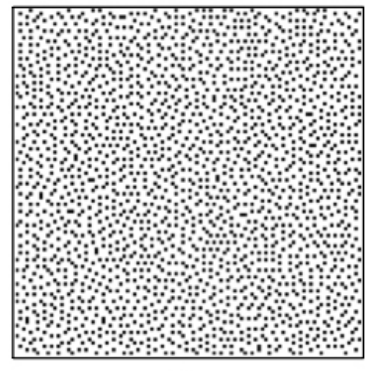

(b)

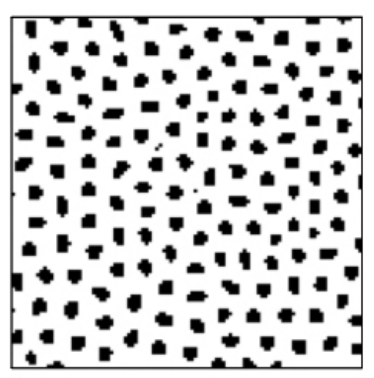

(c)

Figure 8.14: A $20 \%$ halftone patch printed at $100 \mathrm{dpi}$, halftoned by (a) AM halftoning (15 degrees), (b) FM halftoning, (c) FM2nd halftoning.

These results show that, as expected, the FM halftoning when the color separations are halftoned independently is not so much sensitive to register shift. The small $\Delta E_{a b}$ for this halftoning can be partly due to the inevitable measurement errors. The FM2nd is, as expected, more sensitive to register shift but more shifts in position does not necessarily lead to larger $\Delta E_{a b}$ color differences, which is also valid for AM halftoning. Using different angles in AM halftoning has not reduced the color shift in this case. Due to the periodical structure of the halftoned dots in AM halftoning, a smaller shift in position can sometimes lead to a larger color difference than a larger shift. Table 8.1 clearly shows that AM halftoning is very sensitive to register shift and using different angles does not help in all situations as much as one would expect in some cases [36]. It might be added here that one of the reasons why using different angles have not reduced the color shift is that the studied simulated shift was equal in both $\mathrm{X}$ and $\mathrm{Y}$ directions, which should actually have been avoided. Another reason is that these measurements and simulations were based on two colors, but in case of CMYK printing the difference would probably be more significant. 
Table 8.1: The color difference $\Delta E_{a b}$ caused by register shift of 2 and 5 pixels in both $\mathrm{X}$ and $\mathrm{Y}$ directions for different halftonings.

\begin{tabular}{|l|cc|c|c|}
\hline \multirow{2}{*}{ Halftoning Method } & \multicolumn{2}{|c|}{2 pixels $(\mathrm{X}, \mathrm{Y})$} & \multicolumn{2}{c|}{5 pixels $(\mathrm{X}, \mathrm{Y})$} \\
\cline { 2 - 5 } & \multicolumn{2}{|c|}{$\Delta E_{a b}$} & \multicolumn{2}{c|}{$\Delta E_{a b}$} \\
& Average & Maximum & Average & Maximum \\
\hline AM (same angle) & 4.92 & 10.42 & 6.29 & 17.57 \\
AM (different angle) & 5.09 & 10.88 & 4.16 & 12.47 \\
FM & 1.62 & 3.09 & 1.68 & 4.02 \\
FM2nd & 3.11 & 6.04 & 2.65 & 7.83 \\
\hline
\end{tabular}

\subsubsection{Register Shift Measurements}

In order to measure the register shift in color prints, first the method proposed in Section 8.3 is used to separate the two printed color inks. Then an image processing model is used to measure the displacement of the dots in different color separations. Figure 8.15 (a) shows the captured color image of a 10\% AM halftone patch of cyan and magenta printed by commercial offset press (Heidelberg, $1200 \mathrm{dpi}, 150 \mathrm{lpi}$ ) on coated paper, $150 \mathrm{gr} / \mathrm{m}^{2}$. It should be noted that in the digital bitmap the cyan and magenta dots were placed on top of each other (the shift in Figure 8.15 (a) is due to register shift). Figure 8.15 (b) shows the captured image using the interference filter at $700 \mathrm{~nm}$, which means that the visible dots in this figure are cyan. Figure 8.15 (c) shows magenta dots and the shadow of cyan dots using the interference filter at 500 $\mathrm{nm}$. Since the cyan and magenta dots are placed on top of each other

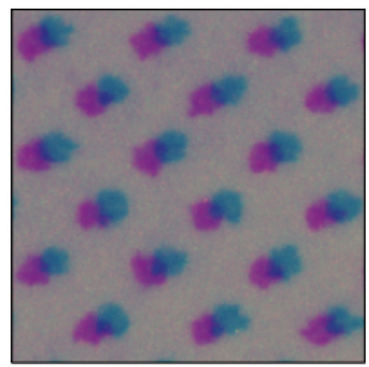

(a)

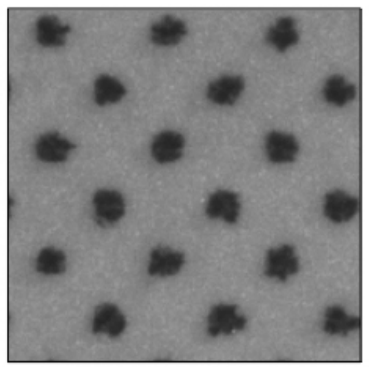

(b)

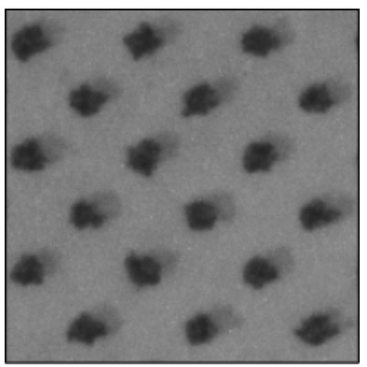

(c)

Figure 8.15: (a) 10\% blue patch Dot-on-Dot in the original bitmap, the shift is due to register shift. (b) Cyan dots. (c) Magenta dots and the shadow of cyan dots. 
in the digital bitmap any shift in position in the final print must have occurred during the printing process. In order to measure the shift we can find the center of the mass for cyan and magenta dots in Figures 8.15 (b) and (c). First by using the MIH approach, the magenta dots are separated from the shadow of cyan in Figure 8.15 (c). By calculating the distance between the centers of the mass of cyan dots and those of magenta dots, the displacement of the dots is measured. Figure 8.16, shows the shifts in pixels in $\mathrm{X}$ and $\mathrm{Y}$ directions for six cyan dots relative to the corresponding magenta dots. Recall that a pixel in the captured images means $1.9 \mu \mathrm{m}$. As can be seen in Figure 8.16 the register shift is around 11 pixels $(20.9 \mu \mathrm{m})$ in the $\mathrm{X}$ direction and around 5 pixels $(9.5 \mu \mathrm{m})$ in the $\mathrm{Y}$ direction. Since the print resolution is $1200 \mathrm{dpi}$, this means that the register shift is around one printed micro dot in $\mathrm{X}$ and a half printed micro dot in $\mathrm{Y}$ direction.

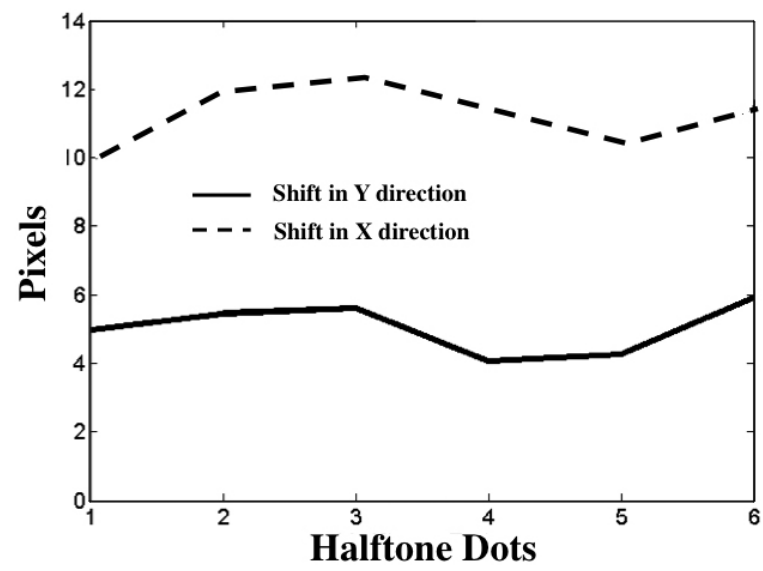

Figure 8.16: The register shift measured in pixels between cyan and magenta dots in $\mathrm{X}$ and $\mathrm{Y}$ directions for six halftone dots.

In order to test the proposed image processing method presented in this section a number of halftone patches of cyan and magenta are created and a shift in the digital bitmap is simulated. The magenta dots are shifted with 5 pixels in both $\mathrm{X}$ and $\mathrm{Y}$ directions. The halftone patches are then printed at 300 dpi using a laser printer (Zerox Phaser, 6180). Then the images are captured by high-resolution camera using a resolution of around $3.25 \mu \mathrm{m} /$ pixel. The cyan and magenta dots are separated from each other and the centers of the mass are found. Since the register shift created by the laser printer at this resolution is neg- 
ligible (which is also verified by capturing the halftone patches with no simulated shift), the measured register shift should actually be the same as the simulated shift. The calculated shift in pixels for both $\mathrm{X}$ and $\mathrm{Y}$ directions were around 130 pixels in the captured images, which means $130 \times 3.25=422.5 \mu \mathrm{m}$. Recall that we had simulated 5 pixels shift, meaning $5 \times 25400 / 300=423.3 \mu \mathrm{m}$ for $300 \mathrm{dpi}$. This test shows that the proposed approach measures the register shift with a very high accuracy.

\subsection{Summary and Discussion}

In this chapter, a method was proposed to separate the effect of the color inks by using microscale images. This method also provides the possibility to investigate the characteristic of the color inks in different printing situations.

The proposed method to separate the physical and optical dot gain was used to investigate the behavior of dot gain in different wavelength bands and for different color inks. Studying the behavior of optical dot gain for different wavelengths, shows that the light scattering in the paper is wavelength independent meaning that it does not cause the optical dot gain to be significantly different at different wavelengths. The reason why the total dot gain differs for color inks at different wavelengths is because of the variation of ink transmittance values in the absorbing wavelength band.

By separating the two primary color inks which are printed on a paper, it is possible to determine the behavior of each color individually. The result of this study can help to improve the accuracy of the existing color prediction models. One great application for this approach is in print electronics. Since in print electronic, materials with different wavelength properties are printed on top of each other, it is possible to separate those layers and detect the possible deficiencies. For example, when a transistor is printed, there are several layers with different wavelength characteristics in the visible wavelength band printed on top of each other. By separating the layers with the proposed approach it is possible to detect the probable invisible disconnection of the lower layers.

In this chapter, a new approach was also presented to measure the register shift. The approach was then examined by measuring the simulated register shift for halftone patches printed by a laser printer. The 
test showed that the proposed approach can measure the register shift with a very good precision. The effect of register shift was then measured by color difference $\Delta E_{a b}$ for different halftoning methods, AM (same angle for cyan and magenta), AM (different angles for cyan and magenta), FM and FM2nd halftoning methods. The results show that both AM methods are very sensitive to register variation for the simulated register shifts, while FM is almost not sensitive at all. In comparison with the AM methods the FM2nd halftoning is also not much sensitive to register shift. 
Chapter 9

\section{Summary and Future Work}

Contents

9.1 Summary . . . . . . . . . . 147

9.2 Future Work . . . . . . . . . . . 148 


\subsection{Summary}

One of the main aims of this dissertation has been to provide new methods for characterizing the halftone prints using the microscopic measurements. Overall this study can help the paper and graphic arts industries to develop their products more efficiently. Investigation of the halftone print properties in microscopic scale requires a high quality image acquisition system to capture the microscale images. This has been accomplished by using a high-resolution camera. The details of the image acquisition system has been described in Chapter 4, which includes the spectral characteristics of the light source, the spectral transmittance of the color filters and the CCD camera.

In order to estimate the total dot gain, three approaches based on Murray-Davies model have been presented and evaluated. One of the approaches was based on the measurements carried out by a spectrophotometer. The other two approaches were based on microscale images captured by the high-resolution camera. It has been shown that all three approaches produce similar results. The fact that the transmitted image incorporates some optical dot gain has also been shown.

The proposed novel MIH approach based on the reflected microscale image histogram estimates the physical dot area of the halftone prints. The MIH approach can be used to estimate the physical dot gain of any halftone print. Separately monitoring physical and optical dot gain can help the paper and graphic art industries to characterize ink dot placement, the ink spreading on the paper and the optical dot gain effects.

To validate the MIH approach, the MTF of eleven paper samples were simulated and compared with measured MTF by knife-edge method, the MTF obtained with Monte-Carlo simulation and the MTF obtained using transmitted images. The results showed that the MTF obtained with MIH applied on reflected image, Monte-Carlo, and knife edge techniques produce similar results.

Another study in this dissertation was to find the dependency of optical dot gain to the shape of the dots by simulating the optical dot gain for different dot shapes produced by different types of halftoning techniques. The optical dot gain was simulated by using the $\mathrm{MTF}_{2 \mathrm{p}}$ proposed by Rogers [99], presented in Chapter 6. The results showed that by increasing the perimeter of the dot shapes, the optical dot gain is increased. However there is a limit to the ratio of perimeter to area 
at which the optical dot gain will be saturated.

The ink behavior and light scattering effect have also been investigated for the dots produced by different halftoning techniques printed on coated and uncoated paper. The three dimensional demonstration of ink has shown that the ink is more spread on the surface of the coated paper compared to the uncoated paper. The results demonstrate that the print property is not only dependent on the optical properties of the paper but also on the geometrical distribution of ink dots on the paper surface.

A new method has been proposed to separate the physical area of the color inks by using microscale images. The method provides the possibility to investigate the characteristic of the color inks in different printing situations.

The behavior of dot gain in different wavelength bands and for different color inks were investigated. The results indicated that the light scattering which is the reason for optical dot gain creation, must be less sensitive to different wavelength bands. Due to the variation of ink transmittance values in the absorbing wavelength band, the total dot gain differs for different wavelengths.

A new approach was also presented to measure the register shift. The results showed that the proposed approach can measure the register shift with a very good precision. The effect of register shift was measured by the color difference $\Delta E_{a b}$ for different halftoning methods. The results showed that AM with same and different angles for cyan and magenta, are very sensitive to register shift and FM2nd is also not much sensitive to register shift compared to AM halftoning technique while FM (first generation) is almost not sensitive at all.

\section{$9.2 \quad$ Future Work}

There are many possible future extensions to the work done in this dissertation. The microscale analysis of halftone prints helps to characterize and understand the halftone color prints. The image acquisition system in our group at Linköping University has still some unexplored flexibilities such as varying the angle of incident illumination, and using the backlight for transmission imaging. The exploration of these flexibilities provide possibilities for future experiments. 
The ink-paper interaction, in general, has a dramatic impact on color reproduction, that deserves more study. However, the study of ink-paper interaction is a very complicated research topic involving experimental measurements and theoretical treatments, demanding knowledge of physics of paper structure, chemistry of the ink, fluid dynamics, ink viscosity, etc. More advanced studies on these topics may result in simulating the physical dot gain, which together with the simulation of optical dot gain presented in this dissertation can predict the total dot gain and thereby the print result prior to the actual printing.

The proposed approach for separating the colors in color print has been limited to two colors. A natural continuation would be to develop the method to handle the case of printed multiple color inks and to analyze the behavior of each ink separately. 


\section{Bibliography}

[1] J. S. Arney, P. G. Engeldrum, and H. Zeng, "A Modified MurrayDavies Model of Halftone Gray Scale", Proceedings of TAGATechnical Association of the Graphic Arts, pp. 353-363, 1995.

[2] J. S. Arney, P. G. Engeldrum, and H. Zeng, "An Expanded MurrayDavies Model of Tone Reproduction in Halftone Imaging", Journal of Imaging Science and Technology, vol. 39, pp. 502-508, 1995.

[3] J. S. Arney, C. D. Arney, and M. Katsube, "An MTF Analysis of Paper", Journal of Imaging Science and Technology, vol. 40, pp. 19-25, 1996.

[4] J. S. Arney, C. D. Arney, M. Katsube, and P. G. Engeldrum, "The Impact of Paper Optical Properties on Hard Copy Image Quality", Proceedings of the $12^{\text {th }}$ International Conference on Digital Printing Technologies, pp. 166-170, 1996.

[5] J. S. Arney, "A Probability Description of the Yule-Nielsen Effect", Journal of Imaging Science and Technology, vol. 41, pp. 633-636, 1997.

[6] J. S. Arney and Y. M. Wong, "Histogram Analysis of the Microstructure of Halftone Images", Proceedings of IS\& T Image Processing, Image Quality, Image Capture, Systems Conference, pp. 206-209, 1998.

[7] J. S. Arney, J. Chauvin, J. Nauman, and P. G. Anderson, "KubelkaMunk Theory and the MTF of Paper", Journal of Imaging Science and Technology, vol. 47, pp. 339-345, 2003.

[8] "Art Workshop: The Munken Guide to Uncoated Paper", www.arcticpaper.com 
[9] R. Balasubramanian and E. Dalal, "A Method for Quantifying the Color Gamut of an Output Device", Proceedings of IS\& T/SPIE, Electronic Imaging Science and Technology, vol. 3018, pp. 110-116, 1997.

[10] R. Balasubramanian, "Optimization of the Spectral Neugebauer Model for Printer Characterization", Journal of Electronic Imaging, vol. 8, pp. 156-166, 1999.

[11] C. E. Barnett, "Some Application of Wavelength Turbidimetry in the Infrared", Journal of Physical Chemistry, vol 46, pp. 6975, 1942.

[12] R. Bartels, "Reducing Patterns in the FM Part of Tile-Based Hybrid Screens", Proceedings of IS\&T Conference, pp. 241-244, 2002.

[13] L. Bergman, "Using Multicoloured Halftone Screens for Offset Print Quality Monitoring", Ph.D. Thesis. Dissertation, No. 1147, Linköping University, Sweden, 2005.

[14] S. Chandrasekhar, Radiative Transfer, Dover Publications Inc., 1960.

[15] W. F. Cheong, S. A. Prahl, and A. J. Welch, "A Review of the Optical Properties of Biological Tissues", IEEE Journal of Quantum Electronics, vol. 26, pp. 2166-2185, 1990.

[16] F. R. Clapper and J. A. Yule, "The Effect of Multiple Internal Reflections on the Densities of Halftone Print on Paper", Journal of the Optical Society of America, vol. 43, pp. 600-603, 1953.

[17] Commission Internationale de l'Eclairage Proceedings, Cambridge: Cambridge University Press, 1931.

[18] L. G. Coppel, P. Edström, and M. Lindquister, "Open Source Monte-Carlo Simulation Platform for Particle Level Simulation of Light Scattering from Generated Paper Structures,", Proceedings of Papermaking Research Symposium Conference, 2009.

[19] L. G. Coppel, M. Neuman, and P. Edström, "Lateral Light Scattering in Paper - MTF Simulation and Measurement", Optics Express, vol. 19, pp. 25181-25187, 2011. 
[20] L. G. Coppel, "Whiteness and Fluorescence in Layered Paper and Board", Ph.D. Thesis. Dissertation, No. 138, Mid Sweden University, 2012.

[21] M. E. Demichel, Le Procédé, vol. 26, pp. 17-21, 1924.

[22] V. Dzimbeg-Malcic, Z. Barbaric-Mikocevic, and K. Itric, "KubelkaMunk Theory in Describing Optical Properties of Paper (I)," Technical Gazette, vol. 18, pp. 117-124, 2011.

[23] Edmond Optics Ltd, Optics and Optical Instruments Catalog, 2003.

[24] P. Emmel and R. D. Hersh, "A Model for Color Prediction of Halftoned Samples Incorporating Light Scattering and Ink Spreading", Proceedings of the IS\& T/SID $7^{\text {th }}$ Color Imaging Conference: Color science, Systems and Applications, pp. 173-181, 1999.

[25] P. Emmel and R. D. Hersch, "Modeling Ink Spreading for Color Prediction", Journal of Imaging Science and Technology, vol. 46, pp. 237-246, 2002.

[26] P.G. Engeldrum, "Color Between the Dots", Journal of Imaging Science and Technology, vol. 38, pp. 545-551, 1994.

[27] P. G. Engeldrum and B. Pridham, "Application of Turbid Medium Theory to Paper Spread Function Measurements", Proceedings of TAGA-Technical Association of the Graphic Arts, vol. 1, pp. 339352, 1995.

[28] P. G. Engeldrum, "Paper Substrate Spread Function and the MTF of Photographic Paper", Journal of Imaging Science and Technology, vol. 48, pp. 50-57, 2004.

[29] M. D. Fairchild, "Colour Appearance Models", Wiley-IS\&T series in Imaging Science and Technology, Second Edition, 2005.

[30] N. P. Garg, A. K. Singla, and R. D. Hersch, "Calibrating the YuleNielsen Modified Spectral Neugebauer Model with Ink Spreading Curves Derived from Digitized RGB Calibration Patch Images", Journal of Imaging Science and Technology, vol. 52, pp. 040908040908-5, 2008. 
[31] S. Gooran, M. Österberg, and B. Kruse, "Hybrid Halftoning - A Novel Algorithm for Using Multiple Halftoning Technologies", Proceedings of IS\& T Conference, pp. 79-86, 1996.

[32] S. Gooran, "High Quality Frequency Modulated Halftoning", Ph.D. Thesis. Dissertation, No. 668, Linköping University, Sweden, 2001.

[33] S. Gooran, "Hybrid Halftoning, A Useful Method for Flexography", Journal of Imaging Science and Technology, vol. 49, pp. 85-95, 2004.

[34] S. Gooran, "Dependent Color Halftoning, Better Quality with Less Ink", Journal of Imaging Science and Technology, vol. 48, pp. 652360, 2004.

[35] S. Gooran, M. Namedanian, and H. Hedman, "A New Approach to Calculate Color Values of Halftone Prints", Proceedings of IARIGAI $36^{\text {th }}$ Research Conference, Advances in Printing and Media Technology, 2009.

[36] S. Gooran, D. Nyström, M. Namedanian, and S. Hauck, "Measuring Register Shift and Investigating its Effect on Color Appearance for Different Halftoning", Proceedings of TAGA-Technical Association of the Graphic Arts, pp. 235-242, 2011.

[37] B. Th. Grande, "Tonal Value Increase in Offset Printing", Technical Report, 2010.

[38] S. Gustavson, "Dot Gain in Color Halftones", Ph.D. Thesis. Dissertation, No. 492, Linköping University, Sweden, 1997.

[39] M. Habekost, "Color Difference Equations and the Human Eye", Proceedings of TAGA-Technical Association of the Graphic Arts, pp. 291-310, 2007.

[40] J. Y. Hardeberg, "Acquisition and Production of Color Images: Colorimetric and Multispectral Approaches", Ph.D. Thesis. Dissertation, ISBN:1-58112-135-0, France, 2001.

[41] S. Hauck and S. Gooran, "An Alternative Method to Determine the Register Variation using Specteophotometry", Proceedings of TAGA-Technical Association of the Graphic Arts, pp. 350-363, 2011. 
[42] Z. He and C. A. Bouman, "AM/FM Halftoning: Digital Halftoning through Simultaneous Modulation of Dot Size and Dot Density", Journal of Electronic Imaging, vol.13, pp. 286-302, 2004.

[43] C. G. Healey, "Effective Visualization of Large Multidimensional Datasets", Ph.D. Thesis. , The University of British Columbia, 1996.

[44] M. Hebert and R. D. Hersch, "Extending the ClapperYule Model through Printing Supports", Journal of the Optical Society of America, vol. 22, pp. 1952-1966, 2005.

[45] M. Hebert and R. D. Hersch, "Reflectance and Transmittance Model for Recto-Verso Halftone Prints", Journal of the Optical Society of America, vol. 23, pp. 2415-2432, 2006.

[46] D. R. Hersch, P. Emmel, F. Collaud, and F. Crete, "Spectral Reflection and Dot Surface Prediction Models for Color Halftone Prints", Journal of Electronic Imaging, vol. 14, pp. 33001-1-33001-12, 2005.

[47] R. D. Hersch and F. Crete, "Improving the Yule-Nielsen Modified Neugebauer Model by Dot Surface Coverages Depending on the Ink Superposition Conditions", IS\& T/SPIE, Electronic Imaging Symposium, vol. 5667, pp. 434-445, 2005.

[48] D. R. Hersch and R. Rossier, "General-Purpose Gamut Mapping", Workshop in $6^{\text {th }}$ European Conference on Color in Graphics, Imaging and Vision, 2012.

[49] P. Le. Hue, "Progress and Trends in Ink-jet Printing Technology", Journal of Imaging Science and Technology, vol. 42, pp. 49-62, 1998.

[50] R. Hunt, "The Reproduction of Color", Kingston-upon-Thames, Fountain Press, Fifth Edition, 1995.

[51] R. Hunt, "Measuring Color", Kingston-upon-Thames, Fountain Press, Third Edition, 1998.

[52] Iggesund Paperboard Reference Manual, Intellecta Infolog, Iggesund Paperboard $A B, 2010$.

[53] S. Inoue, N. Tsumura, and Y. Miyake, "Measuring MTF of Paper by Sinusoidal Test Pattern Projection", Journal of Imaging Science and Technology, vol. 4, pp. 657-661, 1997. 
[54] S. Inoue, "Analyzing CTF of Print by MTF of Paper", Journal of Imaging Science and Technology, vol. 42, pp. 572-576, 1998.

[55] A. Ishimaru, "Wave Propagation and Scattering in Random Media and Rough Surfaces", Proceedings of the IEEE, vol. 79, pp. 13591366, 1991.

[56] ISO 216: "Writing Paper and Certain Classes of Printed Matter; Trimmed Sizes; A and B Series", (International Organization for Standardization: Geneva, Switzerland), 2007.

[57] ISO 2469: " Paper, Board and Pulps-Measurement of Diffuse Reflectance Factor", International Organization for Standardization: Geneva, Switzerland, 2007.

[58] International Lighting Vocabulary. Number 17.4. CIE, 4th edition. ISBN 978-3-900734-07-7, CIE 1987.

[59] E. R. Kandel, J. H. Schwartz, and T. M. Jessell, "Principles of Neural Science", $4^{\text {th }}$ Edition, New York: McGraw-Hill, pp. 507513,2000 .

[60] Ch. Kappel, U. Hirn, M. Donoser, and W. Bauer, "Measurement of Printing Ink Penetration in Uncoated Papers and its Influence on Print Quality", 94 ${ }^{\text {th }}$ Annual meeting pulp and paper technology association of canada, pp. 540-542, 2008.

[61] C. Koopipat, N. Tsumura, M. Fujino, and Y. Miyake, "Effect of Ink Spread and Optical Dot Gain on the MTF of Ink Jet Image", Journal of Imaging Science and Technology, vol. 46, pp. 321-325, 2002 .

[62] C. Koopipat, N. Tsumura, M. Fujino, and Y. Miyake, "Prediction of Image Reflectance Based on the Measurement of Mechanical and Optical Dot Gain", Journal of Japan Hardcopy, vol. 2002, pp. 584$585,2002$.

[63] P. Kubelka and F. Munk, "Ein Beitrag zur Optik der Farbanstriche", Z.Tech. Phys. (Leipzig), vol. 12, pp. 593601, 1931.

[64] P. Kubelka, "New Contributions to the Optics of Intensely LightScattering Materials. Part I", Journal of the Optical Society of America, vol. 38, pp. 448-457, 1948. 
[65] D. L. Lau and G. R. Arce, " Modern Digital Halftoning”, Second Edition, Marcel Dekker, Inc, New York, 2001.

[66] J. Lee and J. P. Allebach, "CMYK Halftoning Algorithm Based on Direct Binary Search", Proceedings of the IS\&T/SID $9^{\text {th }}$ Color Imaging Conference, pp. 199-204, 2001.

[67] D. Malacara, "Color Vision and Colorimetry, Theory and Applications", SPIE Press, Bellingham, Second Edition, 2002.

[68] L. Mandic, S. Grgic, and M. Grgic, "Comparison of Color Difference Equations", Multimedia Signal Processing and Communications, pp. 107-110, 2006.

[69] R. McDonald and K. J. Smith, "CIE94 - A New Color-Difference Formula", Journal of Society of Dyers and Colourists, pp. 376379, 1995.

[70] G. Mie, "Beiträge zur Optik trüber Medien, Speziell Kolloidaler Metallösungen", Annalen der Physik, vierte Folge, vol. 25, pp. 377445, 1908.

[71] P. Mudgett and L. W. Richards, "Multiple Scattering Calculations for Technology", Journal of the Optical Society of America, vol. 10, pp. 1485-1502, 1971.

[72] P. Mudgett and L. W. Richards, "Multiple Scattering Calculations for Technology II ", Journal of Colloid Interface Science, vol. 39, pp. 551-567, 1972.

[73] A. Murray, "Monochrome Reproduction in Photoengraving", Journal of the Franklin Institute, vol. 221, pp. 721-744, 1936.

[74] M. Namedanian and S. Gooran, "High Resolution Analysis of Optical and Physical Dot Gain", Proceedings of TAGA-Technical Association of the Graphic Arts, pp. 48-51, 2010.

[75] M. Namedanian and S. Gooran, "Characterization of Total Dot Gain by Microscopic Image Analysis", Journal of Imaging Science and Technology, vol. 55, pp. 040501-1-040501-7, 2011.

[76] M. Namedanian, S. Gooran, and D. Nyström, "Investigating the Wavelength Dependency of Dot Gain in Color Print", Proceedings 
of IS\&T/SPIE, Electronic Imaging Science and Technology, vol. 7866, pp. 786617-1-786617-8, 2011.

[77] M. Namedanian and S. Gooran, "Characteristic Analysis of the Primary Color Inks in Color Print", Proceedings of IARIGAI, $38^{\text {th }}$ Research Conference, Advances in Printing and Media Technology, pp. 317-322, 2011.

[78] M. Namedanian, L. G. Coppel, M. Neuman, S. Gooran, P. Edström, and P. Kolseth, "Analysis of Optical and Physical Dot Gain by Microscale Image Histogram and Modulation Transfer Functions", Journal of Imaging Science and Technology, vol. 57, pp. 20504-120504-5, 2013.

[79] M. Namedanian and S. Gooran, "Optical Dot Gain Study on Different Halftone Dot Shapes", Proceedings of TAGA-Technical Association of the Graphic Arts, pp. 96-98, 2013.

[80] M. Namedanian, D. Nyström, P. Zitinski Elias, and S. Gooran, "Physical and Optical Dot Gain: Characterization and Relation to Dot Shape and Paper Properties", Accepted to be published in IS\&T/ SPIE, Electronic Imaging Science and Technology, 2014.

[81] K. Nassau, "Color for Science, Art and Technology", Elsevier, pp.355-356, 1997.

[82] H. E. J. Neugebauer, "Die theoretischen grundlagen des mehrfarbendrucks", Zeitscrift fur wissenschaftliche Photographie, vol. 36, pp. 73 89, 1937. [Reprinted as Neugebauer memorial seminar on color reproduction, Proceedings SPIE, vol. 1184, pp. 194 202, 1989.]

[83] M. Neuman and P. Edström, "Anisotropic Reflectance from Turbid Media. I. Theory", Journal of the Optical Society of America, vol. 27, pp. 1032-1039, 2010.

[84] M. Neuman, L. G. Coppel, and P. Edström, "Point Spreading in Turbid Media with Anisotropic Single Scattering", Optics Express, vol. 19, pp. 1915-1920, 2011.

[85] D. Nyström, "Colorimetric and Multispectral Image Acquisition", Licentiate Thesis No. 1289, Linköping University, 2006. 
[86] D. Nyström, "Colorimetric and Multispectral Image Acquisition Using Model-based and Empirical Device Characterization", In B.K. Ersboll \& K.S. Pederson (Eds): Lecture Notes in Computer Science, pp. 798-807, 2007.

[87] D. Nyström, "Reconstructing Spectral and Colorimetric Data Using Trichromatic and Multi-channel Imaging", Proceedings of Ninth International Symposium on Multispectral Color Science and Application, pp. 45-52, 2007.

[88] D. Nyström, "High Resolution Analysis of Halftone Prints", Ph.D. Thesis. Dissertation, No. 1229, Linköping University, Sweden, 2008.

[89] D. Nyström, "A Microscale View on Color Reproduction", Proceedings of IS\&T/SPIE, Fourth European Conference on Color in Graphics, Imaging, and Vision, pp. 542-547, 2008.

[90] D. Nyström, "Physical and Optical Dot Gain: Separation and Relation to Print Resolution", In N. Enlund M. Lovrecek (Eds): Advances in Printing and Media Technology, vol. 36, pp 337-344, 2009.

[91] D. Nyström, "Microscopic Color Measurements of Halftone Prints", Proceedings of NIP26 $-26^{\text {th }}$ International Conference on Digital Printing Technologies, pp. 459-462, 2010.

[92] D. Nyström, "An expanded Neugebauer Formula, Using Varying Micro-Reflectance of the Neugebauer Primaries", Proceedings of CGIV $6^{\text {th }}$ European Conference on Color in Graphics, Imaging, and Vision, pp. 157-162, 2012.

[93] P. Oittinen, "Limits of Microscopic Print Quality", in Advances in Printing Science and Technology, W. H. Banks, vol. 16, pp. 121128, 1982.

[94] M. Pearson, "n-Value for General Conditions", Proceedings of TAGA-Technical Association of the Graphic Arts, pp. 415425, 1980.

[95] Photo Research, PR-650 SpectraScan Spectracolorimeter ${ }^{T M}$ Instruction Manual, Document No. 9903-0006-05 Rev. B, 1999.

[96] http://www.maxlevy.com/productPDF/100MLA0160-001.pdf 
[97] Y. Qu and S. Gooran, "A Simple Color Prediction Model Based on Multiple Dot Gain curves", Proceedings of IS\&T/SPIE, Electronic Imaging Science and Technology, vol. 7866, pp. 786615-1-786615-8, 2011.

[98] G. L. Rogers, "Optical Dot Gain in a Halftone Print: Tone Reproduction and Image Quality in the Graphic Arts", Journal of Imaging Science and Technology, vol. 41, pp. 643-656, 1997.

[99] G. L. Rogers, "Measurement of the Modulation Transfer Function of Paper", Journal of the Optical Society of America, vol. 37, pp. 7235-7240, 1998.

[100] G. L. Rogers, "A Generalized Clapper-Yule Model of Halftone Reflectance", Journal of Color Research and Application, vol. 25, pp. 402-407, 2000.

[101] F. R. Ruckdeschel and O. G. Hauser, "Yule-Nielsen Effect in Printing: A Physical Analysis", Journal of the Optical Society of America, vol. 17, pp. 3376-3383, 1978.

[102] D. L. Schacter, D. T. Gilbert, and D. M. Wegner, "Psychology", New York: Worth Publishers, 2009.

[103] G. Sharma, "Digital Color Imaging", Webster, New York, 2003.

[104] T. Smith and J. Guild, "The C.I.E. Colorimetric Standards and Their Use", Journal of Transactions of the Optical Society, vol. 33, pp. $74-135,1931$.

[105] M. Sormaz, S. Mourad, T. Stamm, and P. Jenny, "Predicting Spectral Halftone Measurements for Different Instruments Using a New Multi-Scale Approach", Proceedings of CGIV 2008/MCS'08 $4^{\text {th }}$ European Conference on Color in Graphics, Imaging, and Vision and $10^{\text {th }}$ international Symposium on Multispectral Color Science (CDROM), IS\&T, pp. 25-30, 2008.

[106] A. P. Stanton and R. D. Warner, "Color Reproduction Characteristics of Stochastically Screened Images", Proceedings of TAGATechnical Association of the Graphic Arts, pp. 65-91, 1994. 
[107] M. Sormaz, T. Stamm, S. Mourad, and P. Jenny, "Stochastic Modeling of Light Scattering with Fluorescence using a MonteCarlo Based Multiscale approach", Journal of the Optical Society of America, vol. 26, pp. 1403-1413, 2009.

[108] M. Ukishima, "Prediction and Evaluation of Color Halftone Print Quality Based on Microscopic Measurement", Ph.D. Thesis. Dissertation, No. 3, University of Eastern, finland, 2010.

[109] M. Ukishima, M. Mäkinen, T. Nakaguchi, N. Tsumura, J. Parkkinen, and Y. Miyake, "A Method to Analyze Preferred MTF for Printing Medium Including Paper", Journal of Springer-Verlag Berlin Heidelberg, LNCS., vol. 5575, pp. 607-616, 2009.

[110] M. Ukishima, H. Kaneko, T. Nakaguchi, N. Tsumura, M. HautaKasari, J. Parkkinen, Y. Miyake, "A Simple Method to Measure MTF of Paper and Its Application for Dot Gain Analysis", IEICE Transactions, vol. 92, pp. 3328-3335, 2009.

[111] UPM Printing Guidelines: Recommendations for prepress and printers, 2004.

[112] J. A. S. Viggiano, "The Color of Halftone Tints", Proceedings of TAGA-Technical Association of the Graphic Arts, pp. 647 661, 1985.

[113] D. Wyble and R. S. Berns, "A Critical Review of Spectral Models Applied to Binary Color Printing", Journal of Color Research and Application, vol. 25, pp. 4-19, 1999.

[114] D. B. Judd and G. Wyszecki, "Colour In Business, Science and Industry", John Wiley Sons, Toronto, 1979.

[115] G. Wyszecki and W. S. Stiles, "Colour Science: Concepts and Methods, Quantitative Data and Formulae", John Wiley and Sons, New York, Second Edition, 2000.

[116] J. Yamashita, H. Sekine, T. Nakaguchi, N. Tsumura, and Y. Miyake, "Spectral Analysis and Modeling of Dot Gain in Inkjet Printing", Journal of The Society of Photographic Science and Technology of Japan, vol. 66, pp. 458-463, 2003. 
[117] L. Yang, R. Lenz, and B. Kruse, "Light Scattering and Ink Penetration Effects on Tone Reproduction", Journal of the Optical Society of America, vol. 18, pp. 360-366, 2001.

[118] L. Yang and B. Kruse, "Revised Kubelka-Munk theory. I. Theory and application", Journal of the Optical Society of America, vol. 21, pp. 1933-1941, 2004.

[119] L. Yang and N. Lundström, "Physical Dot Gain of Offset: Understanding and Determination", Journal of Nordic Pulp \& Paper Research, vol. 22, pp. 388-393, 2007.

[120] J. A. C. Yule and W. J. Nielsen, "The Penetration of Light into Paper and its Effect on Halftone Reproductions", Proceedings of TAGA-Technical Association of the Graphic Arts, pp. 6576, 1951.

[121] J. A. C. Yule, D. J. Howe, and J. H. Altman, "Effect of the Spread Function of Paper on Halftone Reproduction", Tappi, vol. 50, pp. 337-342, 1967. 